\title{
A search for radio jets from massive young stellar objects
}

\section{Association of radio jets with $\mathrm{H}_{2} \mathrm{O}$ and $\mathrm{CH}_{3} \mathrm{OH}$ masers}

\author{
Ü. Kavak ${ }^{1,2,3,4}$, Á. Sánchez-Monge ${ }^{1}$, A. López-Sepulcre ${ }^{5,6}$, R. Cesaroni ${ }^{7}$, F. F. S. van der Tak ${ }^{3,4}$, L. Moscadelli ${ }^{7}$, \\ M. T. Beltrán ${ }^{7}$, and P. Schilke ${ }^{1}$
}

1 I. Physikalisches Institut, Universität zu Köln, Zülpicher Str. 77, 50937 Köln, Germany e-mail: kavak@astro.rug.nl

2 Institute of Graduate Studies in Science, Program of Astronomy and Space Sciences, Istanbul University, Istanbul, Turkey

3 Kapteyn Astronomical Institute, Landleven 12, 9747AD, Groningen, The Netherlands

4 SRON Netherlands Institute for Space Research, Landleven 12, 9747AD, Groningen, The Netherlands

5 Université Grenoble Alpes, CNRS, IPAG, 38000 Grenoble, France

${ }^{6}$ Institut de Radioastronomie Milimétrique (IRAM), 300 rue de la Piscine, F-38406 Saint-Martin-d'Hères, France

7 INAF, Osservatorio Astrofisico di Arcetri, Largo Enrico Fermi 5, I-50125, Florence, Italy

Received February 4, 2020; accepted ??

\section{ABSTRACT}

Context. Recent theoretical and observational studies debate the similarities between the formation process of high-mass (> $\left.8 M_{\odot}\right)$ and low-mass stars. The formation of low-mass star formation is directly associated with the presence of disks and jets. Theoretical models predict that stars with masses up to $140 M_{\odot}$ can be formed through disk-mediated accretion in disk-jet systems. According to this scenario, radio jets are expected to be common in high-mass star-forming regions.

Aims. We aim to increase the number of known radio jets in high-mass star forming regions by searching for radio jet candidates at radio continuum wavelengths.

Methods. We have used the Karl G. Jansky Very Large Array (VLA) to observe 18 high-mass star-forming regions in the C band $\left(6 \mathrm{~cm}, \approx 1^{\prime \prime \prime} 0\right.$ resolution $)$ and $\mathrm{K}$ band $(1.3 \mathrm{~cm}, \approx 0 \prime \prime 3$ resolution $)$. We have searched for radio jet candidates by studying the association of radio continuum sources with shock activity signposts (e.g., molecular outflows, extended green objects, and maser emission). Our VLA observations also target the $22 \mathrm{GHz} \mathrm{H}_{2} \mathrm{O}$ and $6.7 \mathrm{GHz} \mathrm{CH}_{3} \mathrm{OH}$ maser lines.

Results. We have identified 146 radio continuum sources, with 40 of them being located within the field of view of both images ( $\mathrm{C}$ and $\mathrm{K}$ band maps). For these sources we have derived their spectral index, which is consistent with thermal emission (between -0.1 and +2.0 ) for $73 \%$ of them. Based on the association with shock activity signposts, we have identified 28 radio jet candidates. Out these, we have identified 7 as the most probable radio jets. The radio luminosity of the radio jet candidates is correlated with the bolometric luminosity and the outflow momentum rate. About $7-36 \%$ of the radio jet candidates are associated with non-thermal

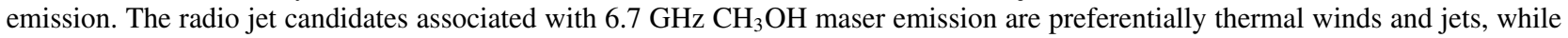
a considerable fraction of radio jet candidates associated with $\mathrm{H}_{2} \mathrm{O}$ masers show non-thermal emission, likely due to strong shocks. Conclusions. About $60 \%$ of the radio continuum sources detected within the field of view of our VLA images are potential radio jets. The remaining sources could be compact $\mathrm{H}_{\text {II }}$ regions in their early stages of development, or radio jets for which we do not have yet further evidence of shock activity. Our sample of 18 regions is divided in 8 less evolved, infrared-dark regions and 10 more evolved, infrared-bright regions. We have found that $\approx 71 \%$ of the identified radio jet candidates are located in the more evolved regions. Similarly, $25 \%$ of the less evolved regions harbor one of the most probable radio jets, while up to $50 \%$ of the more evolved regions contain one of these radio jet candidates. This suggests that the detection of radio jets in high-mass star forming regions is larger in slightly more evolved regions.

Key words. Stars: formation - Stars: massive - ISM: jets and outflows - Radio continuum: ISM - (ISM:) HII Regions

\section{Introduction}

High-mass stars $\left(\mathrm{O}\right.$ and B-type stars with masses $\left.\geq 8 \mathrm{M}_{\odot}\right)$ play a crucial role in the chemical and physical composition of their host galaxies throughout their lifetimes, by injecting energy and material on different scales through energetic outflows, intense UV radiation, powerful stellar winds, and supernova explosions. Despite their importance, the formation process of massive stars is still not well understood due to observational and theoretical challenges (e.g., massive stars form in crowded environments and are located at far distances, see reviews by Tan et al. 2014; Motte et al. 2018). On the other hand, the formation of low-mass stars is better understood and is explained with a model based on accretion via a circumstellar disk and a collimated jet/outflow that removes angular momentum and enables accretion to proceed (e.g., Larson 1969; Andre et al. 2000). Circumstellar disks have been indeed observed around low-mass protostars (-e.g., Williams \& Cieza 2011; Luhman 2012), while ejection of material has been mainly observed by large-scale, collimated jets and outflows (e.g., Bachiller 1996; Bally 2016). For high-mass stars, it remains to be understood the role that (accretion) disks and jets/outflows play in their formation, as well as how their properties vary with the mass of the forming star and the environment. As far as observations are concerned, some studies have concentrated on disks and jets/outflows in selected high-mass star-forming regions (see e.g., Beuther et al. 2002a; Arce et al. 
2007; López-Sepulcre et al. 2009; Bally 2016). The advent of facilities such as the Atacama Large Millimeter/Submillimeter Array (ALMA) or the upgraded Karl G. Jansky Very Large Array (VLA) provides the high spatial resolution and sensitivity necessary to fully resolve the structure of disks and jets/outflows in high-mass star-forming regions. While disks are bright at millimeter wavelengths and constitute perfect targets for ALMA observations (e.g., Sánchez-Monge et al. 2013b, 2014; Beltrán et al. 2014; Johnston et al. 2015; Cesaroni et al. 2017; Maud et al. 2019), jets are found to be bright at centimeter wavelengths observable with the VLA (e.g., Carrasco-González et al. 2010, 2015; Moscadelli et al. 2013, 2016).

Surveys of low-mass star-forming regions with the VLA (e.g., Anglada 1996; Anglada et al. 1998; Beltrán et al. 2001) revealed radio-continuum sources elongated in the direction of the large-scale molecular outflows. These sources are called thermal radio jets, because their emission is interpreted as thermal (freefree) emission of ionised, collimated jets at the base of largerscale optical jets and molecular outflows (e.g., Curiel et al. 1987, 1989; Rodriguez 1995). Due to the high spatial resolution that can be achieved at radio wavelengths with interferometers such as the VLA, thermal radio jets constitute strong evidence of collimated outflows on small scales ( 100 au; Torrelles et al. 1985; Anglada 1996) and permit to pinpoint the location of the star that is forming and powering the jet/outflow seen on larger scales. Although the emission of jets at radio-wavelengths is mainly thermal, some jets show a contribution from a non-thermal component (e.g., Reid et al. 1995; Carrasco-González et al. 2010; Moscadelli et al. 2013, 2016).

Following the strategy used in the study of low-mass starforming regions, we aim to search for radio jets associated with high-mass star-forming regions in a large sample of sources. Until recently, only a limited number of regions harboring highmass stellar objects were known to be associated with radio jets (e.g., HH80/81: Marti et al. 1993; Carrasco-González et al. 2010, CepAHW2: Rodriguez et al. 1994, IRAS 16547-4247: Rodríguez et al. 2008, IRAS 16562-1732: Guzmán et al. 2010, G35.20-0.74 N: Beltrán et al. 2016). In the last years, progress has been made in increasing the number of known jets associated with high-mass young stellar objects (e.g., Moscadelli et al. 2016; Rosero et al. 2016; Sanna et al. 2018; Purser et al. 2018). In this work, we used the VLA in two different frequency bands to search for radio jets in a sample of 18 high-mass star-forming regions associated with molecular outflow emission.

This paper is structured as follows. In Section 2, we present both the sample and the details of the observations. The results of the observations of the radio continuum (and maser) emission are presented in Section 3. The analysis of the properties of the discovered sources is presented in Section 4, while Appendix A describes the properties of each region in more detail. In Section 5, we discuss the implications of our results in the context of high-mass star formation, and in Section 6 we summarize the most important conclusions.

\section{Observations}

\subsection{Selected sample}

We have selected 18 high-mass star-forming regions from the samples of López-Sepulcre et al. (2010, 2011) and SánchezMonge et al. (2013d), using the following criteria: (i) clump mass $>100 \mathrm{M}_{\odot}$, to exclude regions forming mainly low-mass stars, (ii) distance $<4 \mathrm{kpc}$, to resolve spatial scales $<4000 \mathrm{AU}$ when observed with interferometers at a resolution of $1^{\prime \prime}$, (iii) declination $>-15^{\circ}$, to be observable from northern telescopes, (iv) association with an $\mathrm{HCO}^{+}$bipolar outflow and $\mathrm{SiO}$ emission with line widths broader than $>20 \mathrm{~km} \mathrm{~s}^{-1}$ (López-Sepulcre et al. 2011; Sánchez-Monge et al. 2013d), and (v) absence of bright centimeter continuum emission, to exclude developed $\mathrm{H}$ II regions.

We used the NRAO VLA Sky Survey (NVSS; Condon et al. 1998), the MAGPIS (Helfand et al. 2006), CORNISH(Hoare et al. 2012; Purcell et al. 2013), and RMS (Urquhart et al. 2008; Lumsden et al. 2013) surveys to eliminate star-forming regions with developed $\mathrm{H}_{\text {II }}$ regions that would hinder the detection of faint radio jets. Our final sample of 18 high-mass star-forming regions is listed in Table 1.

\subsection{VLA observations}

We used the VLA of the NRAO ${ }^{1}$ to observe the 18 selected regions (see Table 1). The observations were conducted between June and August 2012 (project number 12A-099), when the array was transitioning to its current upgraded phase and was known as 'expanded VLA' (EVLA). During the observations, the array was in its $\mathrm{B}$ configuration, which provides a maximum baseline of $11 \mathrm{~km}$. We observed the frequency bands $\mathrm{C}$ (4-8 GHz) and $\mathrm{K}(18-26.5 \mathrm{GHz})$ with 16 spectral windows of $128 \mathrm{MHz}$ each, covering a total bandwidth of $2048 \mathrm{MHz}$ in each band. Each spectral window has 128 channels, with a channel width of $1 \mathrm{MHz}$. The time spent per source is $\sim 20$ minutes and $\sim 30$ minutes at $6 \mathrm{~cm}$ ( $C$ band) and $1.3 \mathrm{~cm}$ (K band), respectively. Flux calibration was achieved by observing the quasars $3 \mathrm{C} 286\left(F_{1.3 \mathrm{~cm}}=2.59 \mathrm{mJy}, F_{6 \mathrm{~cm}}=7.47 \mathrm{mJy}\right)$ and 3C48 $\left(F_{1.3 \mathrm{~cm}}=1.13 \mathrm{mJy}, F_{6 \mathrm{~cm}}=5.48 \mathrm{mJy}\right)$. The amplitude and phase were calibrated by monitoring the quasars J0555+3948, $\mathrm{J} 0559+2353$, J1832-1035, and J1851+0035. We used the standard guidelines for the calibration of VLA data. The data were processed using the Common Astronomy Software Applications (CASA; McMullin et al. 2007) software.

Continuum images of each source were obtained after excluding channels with line emission, corresponding to $\mathrm{H}_{2} \mathrm{O}$ and $\mathrm{CH}_{3} \mathrm{OH}$ maser lines. The images were done using the 'clean' task with the Briggs weighting parameter set to 2 , which results in a typical synthesized beam of $1^{\prime \prime} .5$ and 0 '.4 for the $\mathrm{C}$ and $\mathrm{K}$ bands, respectively, and typical rms noise levels of $\sim 22 \mu \mathrm{Jy}$ beam $^{-1}$ at $6 \mathrm{~cm}$ and $\sim 30 \mu \mathrm{Jy} \mathrm{beam}^{-1}$ at $1.3 \mathrm{~cm}$ (see Table 1).

The spectral resolution of the observations is limited (about $50 \mathrm{~km} \mathrm{~s}^{-1}$ and $13 \mathrm{~km} \mathrm{~s}^{-1}$ for the $\mathrm{C}$ and $\mathrm{K}$ bands, respectively) and insufficient to resolve spectral features. Despite this limitation, we have produced image cubes of spectral windows that cover the frequencies of the $\mathrm{H}_{2} \mathrm{O}$ maser line at $22235.0798 \mathrm{MHz}$ and the $\mathrm{CH}_{3} \mathrm{OH}$ maser line at $6668.519 \mathrm{MHz}$. This allowed us to search for maser features that can be associated with the continuum emission. The rms noise levels of these cubes are $0.5 \mathrm{mJy} \mathrm{beam}^{-1}$ and $0.3 \mathrm{mJy}^{-1}$ beam $^{-1}$ per channel of 13 and $50 \mathrm{~km} \mathrm{~s}^{-1}$ for the $\mathrm{H}_{2} \mathrm{O}$ and $\mathrm{CH}_{3} \mathrm{OH}$ images, respectively.

1 The Very Large Array (VLA) is operated by the National Radio As-
tronomy Observatory (NRAO), a facility of the National Science Foun-
dation operated under cooperative agreement by Associated Universities, Inc. 
Table 1. High-mass star-forming regions observed with the Very Large Array in this work

\begin{tabular}{|c|c|c|c|c|c|c|c|c|}
\hline \multirow[b]{2}{*}{ Region } & \multirow{2}{*}{$\begin{array}{c}\text { R.A. (J2000) } \\
\text { (h:m:s) }\end{array}$} & \multirow{2}{*}{$\begin{array}{c}\text { Dec. (J2000) } \\
\left({ }^{\circ}:^{\prime \prime}{ }^{\prime \prime}\right)\end{array}$} & \multirow{2}{*}{$\begin{array}{c}d^{a} \\
(\mathrm{kpc})\end{array}$} & \multirow{2}{*}{$\begin{array}{c}M^{a} \\
\left(\mathrm{M}_{\odot}\right)\end{array}$} & \multicolumn{2}{|l|}{$\mathrm{C}$ band $^{b}$} & \multicolumn{2}{|l|}{$\mathrm{K}$ band $^{b}$} \\
\hline & & & & & $\theta_{\text {beam }}, \mathrm{PA}$ & $\mathrm{rms}$ & $\theta_{\text {beam }}, \mathrm{PA}$ & $\mathrm{rms}$ \\
\hline IRAS $05358+3543^{\dagger}$ & $05: 39: 12.2$ & $+35: 45: 52.0$ & 1.8 & 127 & $1.27 \times 1.23,+61$ & 8.1 & $\ldots^{c}$ & \\
\hline $\mathrm{G} 189.78+0.34^{\dagger}$ & 06:08:34.5 & $+20: 38: 51.0$ & 1.8 & 150 & $1.28 \times 1.09,+23$ & 16.0 & c & $\ldots$ \\
\hline G192.58-0.04 & $06: 12: 52.9$ & $+18: 00: 34.9$ & 2.6 & 500 & $1.40 \times 1.19,+21$ & 23.6 & ${ }^{c}$ & \\
\hline G192.60-0.05 & $06: 12: 54.0$ & $+17: 59: 23.0$ & 2.6 & 460 & $1.36 \times 1.14,+20$ & 26.5 & ${ }^{c}$ & $\ldots$ \\
\hline $\mathrm{G} 18.18-0.30^{\dagger}$ & $18: 25: 07.3$ & $-13: 14: 22.9$ & 2.6 & 110 & $1.74 \times 1.05,-16$ & 10.0 & $0.54 \times 0.31,+25$ & 16.7 \\
\hline IRAS $18223-1243^{\dagger}$ & $18: 25: 10.9$ & $-12: 42: 27.0$ & 3.7 & 980 & $1.89 \times 1.14,-14$ & 24.5 & $0.58 \times 0.34,+37$ & 15.0 \\
\hline IRAS $18228-1312^{\dagger}$ & $18: 25: 42.3$ & $-13: 10: 18.0$ & 3.0 & 740 & $1.88 \times 1.16,-14$ & 35.0 & $0.73 \times 0.67,-07$ & 59.1 \\
\hline $\mathrm{G} 19.27+0.1 \mathrm{M} 2^{\ddagger}$ & $18: 25: 52.6$ & $-12: 04: 47.9$ & 2.4 & 114 & $2.08 \times 1.14,-14$ & 9.8 & $0.50 \times 0.32,-26$ & 20.7 \\
\hline $\mathrm{G} 19.27+0.1 \mathrm{M} 1^{*}$ & $18: 25: 58.5$ & $-12: 03: 58.9$ & 2.4 & 113 & $1.95 \times 1.19,-16$ & 9.6 & $0.79 \times 0.42,+49$ & 20.0 \\
\hline IRAS $18236-1205^{\dagger}$ & $18: 26: 25.4$ & $-12: 03: 50.9$ & 2.7 & 780 & $1.99 \times 1.11,-18$ & 10.2 & $0.51 \times 0.32,-26$ & 16.9 \\
\hline $\mathrm{G} 23.60+0.0 \mathrm{M} 1^{*}$ & $18: 34: 11.6$ & $-08: 19: 05.9$ & 2.5 & 365 & $1.85 \times 1.13,-22$ & 8.7 & $0.54 \times 0.30,+32$ & 36.1 \\
\hline IRAS $18316-0602^{\dagger}$ & $18: 34: 20.5$ & $-05: 59: 30.0$ & 3.1 & 1000 & $1.71 \times 1.09,-21$ & 8.4 & $0.53 \times 0.30,+35$ & 40.0 \\
\hline $\mathrm{G} 24.08+0.0 \mathrm{M} 2^{\ddagger}$ & $18: 34: 51.1$ & $-07: 45: 32.0$ & 2.5 & 201 & $1.80 \times 1.13,-21$ & 17.0 & $0.53 \times 0.30,+32$ & 37.0 \\
\hline $\mathrm{G} 24.33+0.1 \mathrm{M} 1 *$ & $18: 35: 07.8$ & $-07: 35: 04.0$ & 3.8 & 1759 & $1.69 \times 1.25,-13$ & 21.0 & $0.49 \times 0.31,-31$ & 26.1 \\
\hline $\mathrm{G} 24.60+0.1 \mathrm{M} 2^{\ddagger}$ & $18: 35: 35.7$ & $-07: 18: 08.9$ & 3.7 & 483 & $1.66 \times 1.03,-20$ & 18.2 & $0.79 \times 0.41,+51$ & 21.8 \\
\hline $\mathrm{G} 24.60+0.1 \mathrm{M} 1^{\ddagger}$ & $18: 35: 40.2$ & $-07: 18: 37.0$ & 3.7 & 192 & $1.67 \times 1.22,-10$ & 12.7 & $0.75 \times 0.38,+57$ & 22.5 \\
\hline $\mathrm{G} 34.43+0.2 \mathrm{M} 3^{\ddagger}$ & $18: 53: 20.3$ & $+01: 28: 23.0$ & 2.5 & 301 & $1.57 \times 1.46,-59$ & 17.9 & $0.53 \times 0.29,+40$ & 19.0 \\
\hline IRAS $19095+0930^{\dagger}$ & 19:11:54.0 & $+09: 35: 52.0$ & 3.0 & 500 & $1.61 \times 1.37,-83$ & 17.0 & $0.53 \times 0.29,+44$ & 63.4 \\
\hline
\end{tabular}

Notes. ${ }^{(a)}$ Distances $(d)$ and clump masses $(M)$ from López-Sepulcre et al. (2011) and Sánchez-Monge et al. (2013d). ${ }^{(b)}$ Synthesized beam $\left(\theta_{\text {beam }}\right)$ major and minor axis in arcsecond, and position angle (PA) in degrees. The rms noise level is given in units of $\mu \mathrm{Jy} \mathrm{beam}^{-1}$. Regions marked with ${ }^{\dagger}$ and ${ }^{\ddagger}$ in the first column indicate IR-loud and IR-dark sources, respectively, based on the classification of López-Sepulcre et al. (2010). ${ }^{(c)}$ Region not observed in the $\mathrm{K}$ band.

Sources detected in the ...

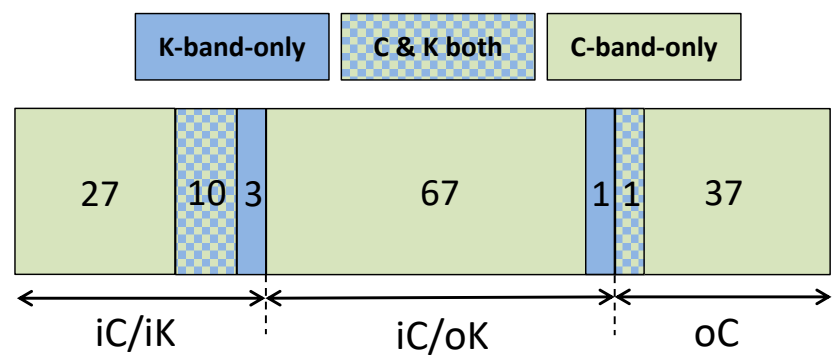

Fig. 1. Number of radio continuum sources detected in the $\mathrm{K}$ band images (marked in blue and corresponding to 15 sources) and in the $\mathrm{C}$ band images (marked in green and corresponding to 142 sources). Out of the 146 detections, only four are detected only in the K band images. The vast majority (131) are detected only in the C band images (see Sect. 3.1 for more details). The bottom labels marked the sources located within the primary beams of the $\mathrm{K}$ band and $\mathrm{C}$ band images. We find 38 sources located outside the $\mathrm{C}$ band primary beam $(\mathrm{oC}), 68$ sources located inside the $\mathrm{C}$ band primary beam but outside the $\mathrm{K}$ band primary beam $(\mathrm{iC} / \mathrm{oK})$ and 40 sources located within the primary beam of both images (iC/iK). See Sects. 3.1 and 3.2 for more details.

\section{Results}

\subsection{Continuum emission}

We have detected compact continuum emissions in all 18 observed high-mass star-forming regions. A total of 146 compact sources are identified with intensities above $3 \sigma$ level, where $\sigma$ is the rms noise level of each map (see Table 1). In Table B.1, we list the coordinates, fluxes and source sizes.

Most of the sources (a total of 131) are only detected in the $\mathrm{C}$ band image, while four of them are only detected in the $\mathrm{K}$ band (see Fig. 1). Only eleven sources are detected at both frequen- cies. The higher detection rate of sources in the $\mathrm{C}$ band is due to several factors. First, four regions were only observed in the $\mathrm{C}$ band (see Table 1). This results in 26 radio continuum sources for which we have no access to $\mathrm{K}$ band images. Second, the field of view of the $\mathrm{C}$ band images (primary beam $\approx 9^{\prime}$ ) is larger compared to the $\mathrm{K}$ band primary beam $\left(\approx 2^{\prime}\right)$. Only 40 sources are located within the $\mathrm{K}$ band primary beam (identified as $\mathrm{iC} / \mathrm{iK}$, see Fig. 1). This number reduces to only 24 when considering only the sources that have been observed in both frequency bands. A total of 68 sources are inside the $\mathrm{C}$ band primary beam (identified as $\mathrm{iC}$ ), but outside the $\mathrm{K}$ band primary beam (identified as oK, see Fig. 1). The remaining 38 sources are outside the primary beam of the $\mathrm{C}$ band observations (marked oC; see also Table B.1). The sources that are outside the primary beam are bright enough to be detected even when the telescope's sensitivity is highly reduced. Thirdly, the spatial filtering of the interferometer is different at both frequencies. In the B-configuration, the VLA recovers scales up to $11^{\prime \prime}$ in the $\mathrm{C}$ band, and only up to $4^{\prime \prime}$ in the $\mathrm{K}$ band (see also Appendix A of Palau et al. 2010). Finally, one cannot exclude the possibility that some of these sources are extragalactic objects that can only be detected at low frequencies. We have followed the approach of Anglada et al. (1998) to determine the possible contamination of background sources in our catalog. The expected number of background sources $N_{\text {bg }}$ is given by

$$
\begin{aligned}
N_{\text {bg }}= & 1.4\left\{1-\exp \left[-0.0066\left(\frac{\theta_{F}}{\operatorname{arcmin}}\right)^{2}\left(\frac{v}{5 \mathrm{GHz}}\right)^{2}\right]\right\} \\
& \times\left(\frac{S_{0}}{\mathrm{mJy}}\right)^{-0.75}\left(\frac{v}{5 \mathrm{GHz}}\right)^{-2.52}
\end{aligned}
$$

where $\theta_{F}$ is the area of the sky that has been observed (18 fields in $\mathrm{C}$ band, and 14 fields in $\mathrm{K}$ band), $v$ is the frequency of the observations, and $S_{0}$ is the detectable flux density threshold 
( $3 \times \mathrm{rms}$, with an average rms of $22 \mu \mathrm{Jy}_{\text {beam }}{ }^{-1}$ in the $\mathrm{C}$ band, and $30 \mu \mathrm{Jy}$ beam $^{-1}$ in the $\mathrm{K}$ band). This results in $N_{\mathrm{bg}}=11$ and $N_{\text {bg }}=0.2$ for the $\mathrm{C}$ and $\mathrm{K}$ band images, respectively. Less than $5 \%$ of the sources detected in the $\mathrm{C}$ band might be background objects not related to the star-forming regions, while we do not expect contamination in the $\mathrm{K}$ band images.

\subsection{Spectral index analysis}

The spectral index $(\alpha)$ is defined as $S_{v} \propto v^{\alpha}$, where $S_{v}$ is the flux density and $v$ is the frequency. We have calculated the spectral index for the continuum sources using the measured flux densities at $1.3 \mathrm{~cm}$ (K band) and $6 \mathrm{~cm}(\mathrm{C}$ band). For the sources without detection in one of the bands, we assume an upper limit of flux density equal to $5 \sigma$. It should be noted here that the flux densities of the sources were corrected for the primary beam response of the antennas. The sources far away from the phase centers (listed in Table 1) have larger uncertainties in the correction factors of the primary beam and thus, in the final (corrected) flux. Therefore, the sources located within the primary beams in both frequency bands (i.e., sources listed as ' $\mathrm{iC} / \mathrm{iK}$ ' in Table B.1) have more accurate flux estimates. For the sources outside one of the primary beams (i.e., oK or oC), we do not determine the spectral index because of the high uncertainty involved in the fluxes. In the last column of Table B.1 we list the calculated spectral indices. For the sources detected at both frequencies, we have improved the determination of the spectral index by creating new images with the same $u v$ (visibility) coverage (see Table B.3). This ensures that the interferometer is sensitive to similar spatial scales at both frequencies.

In Fig. 2, we present the spectral index against the flux density to intensity peak ratio for the 24 continuum sources observed at both bands and located within the primary beams. For the sources detected at $6 \mathrm{~cm}$ only, we derive an upper limit ${ }^{2}$ to the spectral index (see red triangles pointing downwards), while we derive a lower limit for the spectral index for the sources detected only at $1.3 \mathrm{~cm}$, (see blue triangles pointing upwards). The sources detected at both wavelengths (black dots) have a more precise determination of the spectral index. For most sources, we derive spectral indices consistent with thermal emission (i.e., in the range of -0.1 to +2$)$, and in agreement with observations of other radio jets (e.g., Anglada et al. 2018). Only a few sources show very negative spectral indices (\#48, \#96 and \#144). These sources are likely to be partially filtered out in the $\mathrm{K}$ band images, which may result in lower limits for the actual value of the spectral index. In particular, source \#48 appears as three distinguishable peaks, which we refer to as $\mathrm{a}, \mathrm{b}$, and c, surrounded by a more diffuse and extended structure that is mainly visible in the $\mathrm{C}$ band image. It is worth noting that large flux-to-intensity ratios indicate that the source is likely extended and most likely partially filtered out in the $\mathrm{K}$ band images, which may result in negative spectral indices.

\subsection{Maser Emission}

$\mathrm{H}_{2} \mathrm{O}$ and $\mathrm{CH}_{3} \mathrm{OH}$ masers are excellent indicators of star formation activity (e.g., Beuther et al. 2002b; Moscadelli et al. 2005; de Villiers et al. 2015). We created image cubes of the $\mathrm{H}_{2} \mathrm{O}$ and

\footnotetext{
${ }^{2}$ We note that the real spectral index may not be always an upper limit if the source emission is completely filtered out in our $\mathrm{K}$ band images. Further observations at different wavelengths, with a similar $u v$-sampling and angular resolution are necessary to constrain the spectral index of the sources detected only in the $\mathrm{C}$ band images.
}

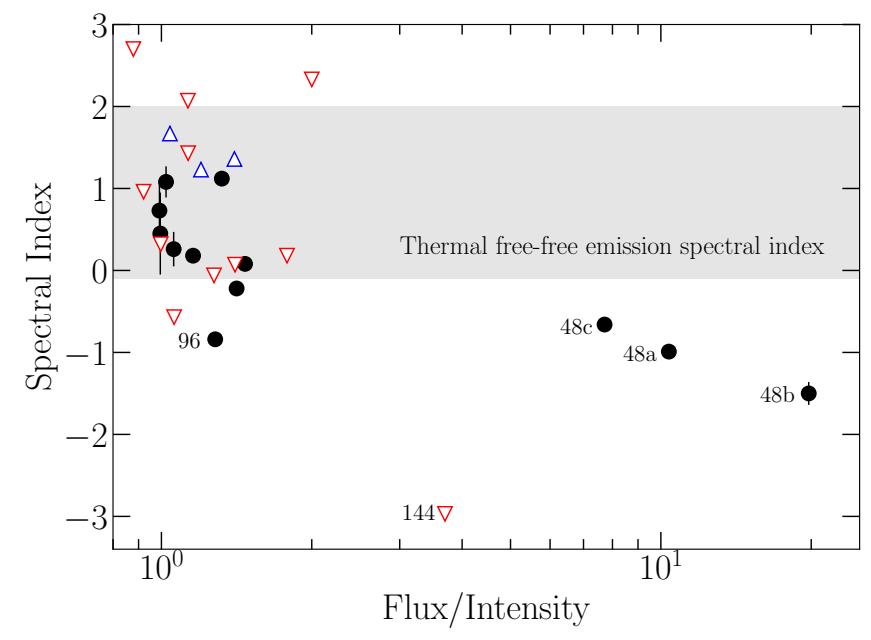

Fig. 2. Spectral index ( $\alpha$, see Sect. 3.2) against the flux to intensity ratio for the radio continuum sources detected in both frequency bands and inside the primary beam of both images (sources listed as ' $\mathrm{iC} / \mathrm{iK}$ ' in Table B.1). The grey-shaded region depicts the spectral index regime associated with thermal free-free emission (i.e., in the range from -0.1 to +2 ). Black dots correspond to sources detected in both bands (see spectral indices in Table 3), blue up-pointing triangles correspond to sources detected only in the $\mathrm{K}$ band (i.e., lower limits), and red downpointing triangles correspond to sources detected only in the $\mathrm{C}$ band (i.e., upper limits).

$\mathrm{CH}_{3} \mathrm{OH}$ spectral lines and searched for maser features by scanning the entire velocity range. Despite the limited spectral resolution of our observation setup (see Sect. 2), we found maser emission in 14 of the 18 regions. In Table 2, we list the coordinates of the maser features detected in each region, together with the velocity at which the feature is detected and its intensity. We also compare the velocities of the maser features with the systemic velocities determined from $\mathrm{H}^{13} \mathrm{CO}^{+}(1-0)$ observations (López-Sepulcre et al. 2011). We find that the $\mathrm{H}_{2} \mathrm{O}$ masers have velocities that match the $\mathrm{H}^{13} \mathrm{CO}^{+}(1-0)$ velocities, while the $\mathrm{CH}_{3} \mathrm{OH}$ masers have a larger discrepancy, probably due to the lower spectral resolution.

The low spectral resolution in our observations compared to the typical maser line widths (a few $\mathrm{km} \mathrm{s}^{-1}$; Elitzur 1982; Kalenskii \& Kurtz 2016) leads to smearing of the maser intensities. The intensities given in Table 2 should be considered as lower limits. Despite this limitation, the high-angular resolution of our observations can be used to spatially associate the $\mathrm{H}_{2} \mathrm{O}$ and $\mathrm{CH}_{3} \mathrm{OH}$ masers to the detected continuum sources. If the angular separation between the continuum source and the maser is smaller than the synthesized beam size (listed in Table 1), we assume that the maser is associated with the continuum source. In the last column of Table 2, we specify the identifier of the continuum source (see Table B.1) with which the maser is associated. We find 10 continuum sources associated with maser features (see Section 4.4 for more details).

\section{Analysis and discussion}

In this section, we determine how many sources in our sample are potential radio jets. For this purpose we study the nature of the detected radio continuum emission, and investigate the association with molecular outflows, masers and EGOs ${ }^{3} / \mathrm{IRAC}$

\footnotetext{
3 The so-called EGOs (extended green objects) are sources with bright emission in the Spitzer
} 
Table 2. $\mathrm{H}_{2} \mathrm{O}$ and $\mathrm{CH}_{3} \mathrm{OH}$ maser features

\begin{tabular}{|c|c|c|c|c|c|c|c|}
\hline Region & Maser & $\begin{array}{l}\text { R.A. (J2000) } \\
\text { (h:m:s) }\end{array}$ & $\begin{array}{c}\text { Dec. (J2000) } \\
\left({ }^{\prime} \vdots^{\prime \prime}\right)\end{array}$ & $\begin{array}{c}V_{\text {maser }}^{\mathrm{a}} \\
\left(\mathrm{km} \mathrm{s}^{-1}\right)\end{array}$ & $\begin{array}{c}V_{\mathrm{LSR}}^{\mathrm{H}^{13} \mathrm{CO}^{+}} \\
\left(\mathrm{km} \mathrm{s}^{-1}\right)\end{array}$ & $\begin{array}{c}\text { Intensity } \\
\left(\text { Jy beam }^{-1}\right)\end{array}$ & $\begin{array}{l}\text { Continuum } \\
\text { source ID }\end{array}$ \\
\hline IRAS $05358+3543$ & $\mathrm{CH}_{3} \mathrm{OH}$ & $05: 39: 13.071$ & $+35: 45: 50.938$ & -304 & -15.8 & 0.028 & 2 \\
\hline G189.78+0.34 & $\mathrm{CH}_{3} \mathrm{OH}$ & 06:08:35.304 & $+20: 39: 06.405$ & -13 & +9.2 & 0.014 & 14 \\
\hline G192.58-0.04 & $\mathrm{CH}_{3} \mathrm{OH}$ & $06: 12: 54.026$ & $+17: 59: 23.060$ & -14 & +9.1 & 0.72 & 22 \\
\hline G18.18-0.30 & $\mathrm{H}_{2} \mathrm{O}$ & $18: 25: 07.575$ & $-13: 14: 31.487$ & -3 & +50.0 & 0.57 & - \\
\hline IRAS $18223-1243$ & $\mathrm{H}_{2} \mathrm{O}$ & $18: 25: 10.804$ & $-12: 42: 26.234$ & +24 & +45.2 & 0.006 & - \\
\hline IRAS 18228-1312 & $\mathrm{H}_{2} \mathrm{O}$ & $18: 25: 41.935$ & $-13: 10: 19.591$ & +24 & +33.1 & 0.022 & 48 \\
\hline \multirow[t]{5}{*}{ IRAS $18236-1205$} & $\mathrm{H}_{2} \mathrm{O}$ & $18: 26: 25.677$ & $-12: 03: 48.402$ & +28 & +26.5 & 0.010 & 63 \\
\hline & $\mathrm{H}_{2} \mathrm{O}$ & $18: 26: 25.575$ & $-12: 03: 48.502$ & +28 & +26.5 & 0.006 & 63 \\
\hline & $\mathrm{H}_{2} \mathrm{O}$ & $18: 26: 25.782$ & $-12: 03: 53.263$ & +15 & +26.5 & 0.010 & 64 \\
\hline & $\mathrm{H}_{2} \mathrm{O}$ & $18: 26: 27.149$ & $-12: 03: 54.888$ & +15 & +26.5 & 0.014 & - \\
\hline & $\mathrm{CH}_{3} \mathrm{OH}$ & $18: 26: 25.788$ & $-12: 03: 53.456$ & +5 & +26.5 & 0.26 & 64 \\
\hline G19.27+0.1M1 & $\mathrm{H}_{2} \mathrm{O}$ & $18: 25: 58.546$ & $-12: 03: 58.516$ & +28 & +26.5 & 0.022 & - \\
\hline \multirow[t]{2}{*}{$\mathrm{G} 23.60+0.0 \mathrm{M} 1$} & $\mathrm{H}_{2} \mathrm{O}$ & $18: 34: 11.237$ & $-08: 19: 07.680$ & +108 & +106.5 & 0.44 & - \\
\hline & $\mathrm{H}_{2} \mathrm{O}$ & $18: 34: 11.452$ & $-08: 19: 07.138$ & +108 & +106.5 & 0.10 & - \\
\hline \multirow[t]{2}{*}{ IRAS 18316-0602 } & $\mathrm{H}_{2} \mathrm{O}$ & $18: 34: 20.918$ & $-05: 59: 41.638$ & +41 & +42.5 & 11.1 & 83 \\
\hline & $\mathrm{CH}_{3} \mathrm{OH}$ & $18: 34: 20.913$ & $-05: 59: 42.087$ & -233 & +42.5 & 0.014 & 83 \\
\hline \multirow[t]{2}{*}{$\mathrm{G} 24.33+0.1 \mathrm{M} 1$} & $\mathrm{H}_{2} \mathrm{O}$ & $18: 35: 08.123$ & $-07: 35: 04.216$ & +108 & +113.6 & 4.03 & 110 \\
\hline & $\mathrm{CH}_{3} \mathrm{OH}$ & $18: 35: 08.147$ & $-07: 35: 04.260$ & -182 & +113.6 & 0.010 & 110 \\
\hline $\mathrm{G} 24.60+0.1 \mathrm{M} 2$ & $\mathrm{H}_{2} \mathrm{O}$ & $18: 35: 35.728$ & $-07: 18: 08.796$ & +122 & +115.3 & 0.031 & - \\
\hline $\mathrm{G} 24.60+0.1 \mathrm{M} 2$ & $\mathrm{H}_{2} \mathrm{O}$ & $18: 35: 40.120$ & $-07: 18: 37.417$ & +54 & +53.2 & 1.02 & 136 \\
\hline \multirow[t]{3}{*}{ IRAS $19095+0930$} & $\mathrm{H}_{2} \mathrm{O}$ & $19: 11: 53.975$ & $+09: 35: 50.559$ & +37 & +43.9 & 26.8 & 143 \\
\hline & $\mathrm{H}_{2} \mathrm{O}$ & $19: 11: 53.990$ & $+09: 35: 49.848$ & +37 & +43.9 & 10.3 & 143 \\
\hline & $\mathrm{CH}_{3} \mathrm{OH}$ & 19:11:53.993 & $+09: 35: 50.641$ & +39 & +43.9 & 0.043 & 143 \\
\hline
\end{tabular}

Notes. ${ }^{(a)}$ Uncertainties in the reported maser velocities $\left(V_{\text {maser }}\right)$ are expected to be $\sim 50 \mathrm{~km} \mathrm{~s}^{-1}$ for the $\mathrm{CH}_{3} \mathrm{OH}$ masers and $\sim 13 \mathrm{~km} \mathrm{~s}{ }^{-1}$ for the $\mathrm{H}_{2} \mathrm{O}$ masers (see Sect. 2). The systemic velocities $\left(V_{\mathrm{LSR}}^{\mathrm{H}^{13} \mathrm{CO}^{+}}\right)$are reported in López-Sepulcre et al. (2011). ${ }^{(b)}$ Radio continuum source spatially associated with the maser feature and listed as identified in Table B.1.

$4.5 \mu \mathrm{m}$ band and are usually found associated with strong shocks and jets (e.g., Cyganowski et al. 2008, 2009). By using these criteria, we identify the best radio jet candidates in our sample and characterize their properties.

\subsection{Nature of the radio continuum emission}

Usually two mechanisms are invoked to explain the origin of thermal free-free radiation from ionized gas in star-forming regions: Photoionization and ionization through shocks (e.g., Gordon \& Sorochenko 2002; Kurtz 2005; Sánchez-Monge et al. 2008, 2013c; Anglada et al. 2018). In the case of photoionization, ultraviolet (UV) photons with energies above $13.6 \mathrm{eV}$ are emitted by massive stars and ionize the surrounding atomic hydrogen. In the second scenario, the ionization is produced when ejected material associated with outflows and jets interacts in a shock with neutral and dense material surrounding the forming star (e.g., Curiel et al. 1987, 1989; Anglada et al. 1992).

Anglada $(1995,1996)$ show that the relation between the radio luminosity and the bolometric luminosity of young stellar objects (YSOs) depends on the origin of the ionization: stellar UV radiation or shocks (see also Anglada et al. 2018). We use this relation to investigate the nature of our continuum sources. The solid line in Fig. 3 shows the maximum radio luminosity that a high-mass object of a given luminosity may have according to its UV radiation, the so-called Lyman continuum limit usually associated with $\mathrm{H}$ II regions. The radio luminosity decreases fast with decreasing bolometric luminosity. In contrast, the radio luminosity originated in shocks (i.e., radio jets) has a less steep curve. The dotted line in Fig. 3 shows the least-squares fit to the sample of radio jets studied in Anglada et al. (2018) and shown as grey squares in the figure.

We have calculated the radio luminosity of our continuum sources as $L_{\text {radio }}=S_{v} d^{2}$, where $S_{v}$ is the observed flux density in the $\mathrm{C}$ band (listed in Table B.1) and $d$ is the distance to the source (listed in Table 1$)$. The bolometric luminosity $\left(L_{\mathrm{bol}}\right)$ of each source is uncertain due to the lack of high-resolution data at far-infrared wavelengths. The bolometric luminosity of each region is given in Table A.1 of López-Sepulcre et al. (2011) and provides an upper limit to the actual luminosity. As a simple approach, we divide the bolometric luminosity by the number of radio sources detected within the primary beam to have an estimate of the expected average luminosity for the continuum sources in the region. Circle symbols in Fig. 3 show the continuum sources detected in our work and located inside the $\mathrm{K}$ band primary beam (i.e., with reliable flux measurements and listed as $\mathrm{iC} / \mathrm{iK}$ in $\mathrm{Ta}-$ ble B.1). Colored symbols correspond to those sources for which we could derive the spectral index (see Fig. 2), with blue symbols corresponding to positive spectral indices (i.e., $\alpha>+0.0$, mainly thermal emission) and red symbols corresponding to negative spectral indices. In general, our sources lie in between the two lines defining the radio jet and $\mathrm{H}_{\text {II }}$ region regimes ${ }^{4}$. Interestingly, sources with positive spectral indices (blue symbols) seem to preferentially follow, although with some dispersion, the relation found for radio jets, while sources with negative spectral indices (red symbols) are located closer to the Lyman

\footnotetext{
${ }^{4}$ Some sources lie above the solid curve depicting the Lyman continuum limit. This is in agreement with other studies that report the existence of a population of $\mathrm{H}_{\text {II }}$ regions with radio fluxes larger than the Lyman continuum limit (see e.g., Sánchez-Monge et al. 2013a; Cesaroni et al. 2016).
} 


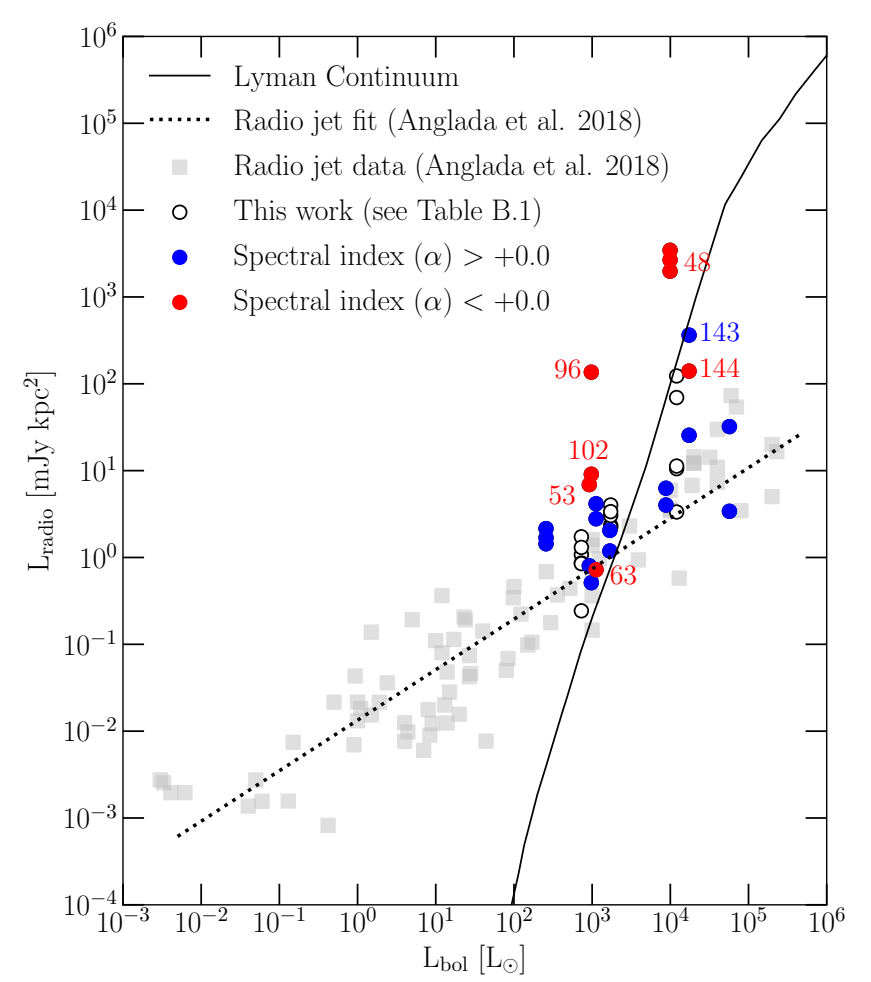

Fig. 3. Scatter plot of bolometric luminosity $\left(L_{\mathrm{bol}}\right)$ and observed radio continuum luminosity $\left(L_{\text {radio }}\right)$ at $6 \mathrm{~cm}$ (C band). Open black circles correspond to the continuum sources detected in our work and located within the primary beam of the $\mathrm{K}$ band $(1.3 \mathrm{~cm})$ images (i.e., ' $\mathrm{iC} / \mathrm{iK}$ ' in Table B.1). Blue and red symbols mark the sources with positive and negative spectral indices, respectively, as listed in Table B.1 and shown in Fig. 2). The solid line represents the values expected from Lyman continuum radiation for a zero-age main-sequence star of a given luminosity (Thompson 1984). The dashed line is a least-squares fit to the radio jets reported by Anglada et al. (2018, shown as grey squares), corresponding to $\left[L_{\text {radio }} / \mathrm{mJy} \mathrm{kpc}^{2}\right]=10^{-1.90}\left[L_{\mathrm{bol}} / L_{\odot}\right]^{+0.59}$ (see their Eq. 28 ).

continuum regime. This favours our previous interpretation that sources with negative spectral indices may be slightly extended $\mathrm{H}$ II regions that are partially filtered out in the $\mathrm{K}$ band images.

\subsection{Association with molecular outflows}

We investigate the association of radio continuum sources with molecular outflows by comparing the location of radio sources with respect to the molecular outflow emission reported mainly by López-Sepulcre et al. (2010) and Sánchez-Monge et al. (2013d). It is expected that the most promising radio jet candidates will be in the center of the molecular outflow emission.

We find a total of twenty-four radio continuum sources that are spatially associated with molecular outflow emission (see Table 3 and Fig. 4 for more details). Out of these sources, eighteen (\#2, \#4, \#13, \#14, \#15, \#16, \#22, \#23, \#25, \#48a, \#48b, \#48c, $\# 74, \# 83$, \#95, \#110, \#137 and \#143) are located at or near the geometric center of the molecular outflow emission, while the remaining six (\#12, \#63, \#64, \#65, \#73, and \#144) are located within the outflow lobes. Although we cannot confirm that these six sources are at the base of the outflows detected with singledish telescopes (with angular resolutions of 11-29"), we cannot exclude that they might drive molecular outflows. Further observations of outflow tracers at higher angular resolution are necessary to confirm and better associated the molecular outflows with the radio continuum sources. In Table 3, we list the outflow momentum rates reported in the literature (see López-Sepulcre et al. 2010; Sánchez-Monge et al. 2013d). For \#137, no outflow momentum rate has been reported (Hatchell et al. 2001; Liu et al. 2013).

\subsection{Association with EGOs}

In this section, we investigate the association of radio continuum sources with Spitzer/IRAC $4.5 \mu$ m emission tracing EGOs, which are considered related to the shocked gas. For the association with EGOs we used the catalogues of Cyganowski et al. (2008, 2009). In total, we found six sources (\#42, \#63, \#64, $\# 119, \# 137$ and \#139) with an EGO counterpart (see Table 3).

We have also inspected the Spitzer/IRAC images of the different regions to search for other possible EGOs not included in previous catalogues. We have identified nine radio continuum sources in this category (see sources \#48, \#65, \#73, \#74, \#83, $\# 110$, and \#143, marked with a '?' symbol in Table 3). The association of these sources with bright $4.5 \mu \mathrm{m}$ emission suggests their association with strong shocks and favors the hypothesis of a radio jet origin for the radio continuum emission of these objects. However, a more detailed characterization of the infrared properties of the nine additional sources is necessary to confirm whether these objects are EGOs or not.

\subsection{Association with masers}

Our VLA observations (see Sect. 2.2) allow us to search for $\mathrm{H}_{2} \mathrm{O}$ and $\mathrm{CH}_{3} \mathrm{OH}$ maser spots associated with radio continuum sources. As shown in Table 2, we have found a total of sixteen $\mathrm{H}_{2} \mathrm{O}$ and seven $\mathrm{CH}_{3} \mathrm{OH}$ maser spots.

We find ten radio continuum sources associated with maser features, of which three (\#2,\#14 and \#22) are associated with $\mathrm{CH}_{3} \mathrm{OH}$ masers only, three sources (\#48, \#63, and \#136) are associated only with $\mathrm{H}_{2} \mathrm{O}$ masers, and four sources (\#64, \#83, \#110 and \#143) are associated with both types of masers (see Table 3 and Fig. 4). It is worth noting that the three sources associated only with $\mathrm{CH}_{3} \mathrm{OH}$ masers correspond to regions observed only in the $\mathrm{C}$ band. Future observations of these sources in the $\mathrm{K}$ band together with observations of the $\mathrm{H}_{2} \mathrm{O}$ maser line could confirm that all sources associated with $\mathrm{CH}_{3} \mathrm{OH}$ maser are also associated with $\mathrm{H}_{2} \mathrm{O}$ maser features.

The observed Class II $6.7 \mathrm{GHz} \mathrm{CH}_{3} \mathrm{OH}$ masers are ideal signposts for embedded young stellar objects and mark the location of deeply embedded massive protostars (e.g., Breen et al.

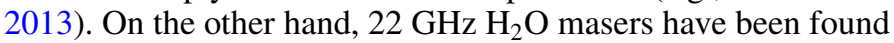
associated with outflow activity (e.g., Torrelles et al. 2011) as well as tracing disk-like structures around young stellar objects (e.g., Moscadelli et al. 2019). Our maser observations have therefore enabled us to identify at least seven potential candidates for a radio jet (i.e., sources associated with outflow activity).

\subsection{Radio jet candidates}

Out of the 146 radio continuum sources detected in our study, we have identified twenty-eight sources (see list at the beginning of Table 3) as possible radio jet candidates, based on their association with outflow and shock activity. We find twenty-four of these sources associated with molecular outflow emission, six of them with EGOs, and ten with masers. In the sketch presented in Fig. 4, we summarize these findings. 
Table 3. Properties of the radio jet candidates.

\begin{tabular}{|c|c|c|c|c|c|c|c|c|c|}
\hline \multirow[b]{2}{*}{ ID } & \multicolumn{3}{|c|}{ Flux properties $^{a}$} & \multicolumn{3}{|c|}{ Source size properties ${ }^{b}$} & \multicolumn{3}{|c|}{ Outflow/shock activity ${ }^{c}$} \\
\hline & $S_{\mathrm{C} \text { band }}$ & $S_{\mathrm{K} \text { band }}$ & $\alpha$ & $\theta_{\mathrm{C} \text { band }}$ & $\theta_{\mathrm{K} \text { band }}$ & $\beta$ & $\log \left(\dot{P}_{\text {out }}\right)$ & EGOs & Masers \\
\hline \multicolumn{10}{|c|}{ Radio jet candidates with signposts of outflow activity } \\
\hline $4^{d}$ & $0.33 \pm 0.01$ & - & - & 1.23 & - & - & -3.9 & $\mathrm{n}$ & $\ldots$ \\
\hline $12^{d}$ & $0.72 \pm 0.03$ & - & - & 0.97 & - & - & $-3.1^{\dagger}$ & $\mathrm{n}$ & $\ldots$ \\
\hline $13^{d}$ & $1.24 \pm 0.05$ & - & - & 0.75 & - & - & -3.1 & $\mathrm{n}$ & $\ldots$ \\
\hline $16^{d}$ & $1.04 \pm 0.04$ & - & - & 1.16 & - & - & -3.1 & $\mathrm{n}$ & $\ldots$ \\
\hline $22^{d}$ & $10.27 \pm 0.22$ & - & - & 1.55 & - & - & -3.3 & $\mathrm{n}$ & $\mathrm{CH}_{3} \mathrm{OH}$ \\
\hline $23^{d}$ & $1.56 \pm 0.05$ & - & - & $\ldots$ & - & - & -3.3 & $\mathrm{n}$ & $\ldots$ \\
\hline $25^{d}$ & $0.49 \pm 0.02$ & - & 一 & $\ldots$ & - & - & -3.3 & $\mathrm{n}$ & $\ldots$ \\
\hline 42 & $4.16 \pm 0.08$ & $\ldots$ & $\ldots$ & 0.81 & $\ldots$ & $\ldots$ & $\ldots$ & $\mathrm{Y}$ & $\ldots$ \\
\hline $64^{e}$ & $0.28 \pm 0.02$ & $0.35 \pm 0.08$ & $+0.18 \pm 0.04$ & $<1.38$ & 0.66 & $>-0.56$ & $-2.6^{\dagger}$ & Y & $\mathrm{H}_{2} \mathrm{O}, \mathrm{CH}_{3} \mathrm{OH}$ \\
\hline $65^{e}$ & $0.57 \pm 0.14$ & $2.31 \pm 0.09$ & $+1.08 \pm 0.19$ & $<1.46$ & $<0.75$ & $\ldots$ & $-2.6^{\dagger}$ & $?$ & $\ldots$ \\
\hline $73^{e}$ & $0.24 \pm 0.15$ & $0.43 \pm 0.09$ & $+0.45 \pm 0.50$ & 0.38 & $<0.74$ & $<+0.51$ & $-2.4^{\dagger}$ & $?$ & $\ldots$ \\
\hline $74^{e}$ & $0.35 \pm 0.03$ & $0.49 \pm 0.13$ & $+0.26 \pm 0.21$ & 0.53 & 0.42 & -0.18 & -2.4 & $?$ & $\ldots$ \\
\hline $83^{e}$ & $3.35 \pm 0.21$ & $3.73 \pm 0.29$ & $+0.08 \pm 0.08$ & 0.87 & 0.85 & -0.02 & -1.8 & $?$ & $\mathrm{H}_{2} \mathrm{O}, \mathrm{CH}_{3} \mathrm{OH}$ \\
\hline 95 & $<0.042$ & $0.25 \pm 0.07$ & $>+1.36$ & $\ldots$ & $<0.42$ & $\ldots$ & -1.8 & $\mathrm{n}$ & $\ldots$ \\
\hline $110^{e}$ & $0.46 \pm 0.21$ & $1.20 \pm 0.06$ & $+0.73 \pm 0.35$ & $<1.39$ & 0.37 & $>-1.01$ & -2.9 & $?$ & $\mathrm{H}_{2} \mathrm{O}, \mathrm{CH}_{3} \mathrm{OH}$ \\
\hline 119 & $1.11 \pm 0.06$ & $<0.022$ & $\ldots$ & 2.28 & $\ldots$ & $\ldots$ & $\ldots$ & Y & $\ldots$ \\
\hline 136 & $<0.10$ & $0.85 \pm 0.12$ & $>+1.67$ & $\ldots$ & 1.50 & $\ldots$ & $\ldots$ & $\mathrm{n}$ & $\mathrm{H}_{2} \mathrm{O}$ \\
\hline $137^{e}$ & $14.40 \pm 1.20$ & $2.21 \pm 0.20$ & $\ldots$ & 2.53 & 1.60 & -0.35 & $\ldots{ }^{*}$ & Y & $\ldots$ \\
\hline 139 & $0.73 \pm 0.03$ & $<0.019$ & $\ldots$ & 0.87 & $\ldots$ & $\ldots$ & $\ldots$ & $\mathrm{Y}$ & $\ldots$ \\
\hline
\end{tabular}

Radio continuum sources consistent with positive spectral index, but with no signposts of outflow activity

\begin{tabular}{|c|c|c|c|c|c|c|c|c|c|}
\hline 61 & $0.14 \pm 0.03$ & $<2.10$ & $<+2.07$ & 1.79 & . & $\ldots$ & $\cdots$ & $\mathrm{n}$ & $\cdots$ \\
\hline 62 & $<0.05$ & $0.24 \pm 0.09$ & $>+1.23$ & $\ldots$ & $<0.44$ & $\ldots$ & $\ldots$ & $\mathrm{n}$ & $\ldots$ \\
\hline 86 & $0.35 \pm 0.01$ & $<2.32$ & $<+1.43$ & $<1.45$ & $\cdots$ & $\cdots$ & $\ldots$ & $\mathrm{n}$ & $\cdots$ \\
\hline 109 & $0.08 \pm 0.03$ & $<1.75$ & $<+2.33$ & $<0.94$ & $\ldots$ & $\ldots$ & $\ldots$ & $\mathrm{n}$ & $\ldots$ \\
\hline 113 & $0.28 \pm 0.01$ & $<0.31$ & $<+0.07$ & 0.85 & $\ldots$ & $\ldots$ & $\ldots$ & $\mathrm{n}$ & $\ldots$ \\
\hline 126 & $0.11 \pm 0.01$ & $<0.37$ & $<+0.96$ & $<1.37$ & $\ldots$ & $\ldots$ & $\ldots$ & $\mathrm{n}$ & $\ldots$ \\
\hline 129 & $0.16 \pm 0.01$ & $<0.20$ & $<+0.18$ & 1.23 & $\ldots$ & $\ldots$ & $\ldots$ & $\mathrm{n}$ & $\ldots$ \\
\hline 145 & $2.84 \pm 0.02$ & $<4.34$ & $<+0.32$ & $<1.48$ & $\ldots$ & $\ldots$ & $\ldots$ & $\mathrm{n}$ & $\ldots$ \\
\hline
\end{tabular}

Notes. ${ }^{(a)}$ Primary beam corrected fluxes in mJy as listed in Table B.1. For sources \#42, \#119, \#137 and \#139 it was not possible primary beam correct the fluxes at both bands (see Sect 3), resulting in not usable spectral indices. The spectral index $\alpha$ is defined in Eq. 2. ${ }^{(b)}$ Source sizes in arcsec determined as $\sqrt{\theta_{\text {major }} \times \theta_{\text {minor }}}$, with $\theta_{\text {major }}$ and $\theta_{\text {minor }}$ listed in Table B.2. Upper limits corresponds to sources for which we could not determine a deconvolved source size. The source size index $\beta$ is defined in Eq. 2. ${ }^{(c)}$ Association of the radio continuum source with outflow and shock activity. The associations correspond to: (i) Molecular outflows, with the outflow momentum rate $\dot{P}_{\text {out }}$ given in units of $M_{\odot} \mathrm{yr}^{-1} \mathrm{~km} \mathrm{~s}^{-1}$ (from López-Sepulcre et al. 2010; Sánchez-Monge et al. 2013d), with the symbol ${ }^{\dagger}$ indicating those radio continuum sources located within the outflow lobes and not at the center of the outflow, (ii) EGOs (or extended green objects), based on the catalogue of Cyganowski et al. (2008, question mark symbols indicate the presence of bright Spitzer/IRAC $4.5 \mu \mathrm{m}$ emission although without confirmation of the object being an EGO), and (iii) $\mathrm{H}_{2} \mathrm{O}$ and $\mathrm{CH}_{3} \mathrm{OH}$ masers, as listed in Table 2. Source \#137, marked with a symbol, is associated with molecular outflow emission (Hatchell et al. 2001; Liu et al. 2013), but no outflow momentum rate has been reported. ${ }^{(d)}$ Sources not observed in the K band. For these sources we do not have information on the $\mathrm{K}$ band flux and presence of $\mathrm{H}_{2} \mathrm{O}$ masers. ${ }^{(e)}$ Sources detected at both frequency bands and for which we have created new images using a common $u v$-range that allows us to sample similar spatial scales. Fluxes and source sizes for these sources are taken from Table B.3. Fluxes for source \#137 can not be primary beam corrected and are not usable for spectral index determination.

In addition to these twenty-eight sources, we have also identified eight radio-continuum sources with spectral indices consistent with thermal emission (see bottom list in Table 3). Based on the results shown in Fig. 3, these sources could also be radio jet candidates, despite their lack of association with tracers 


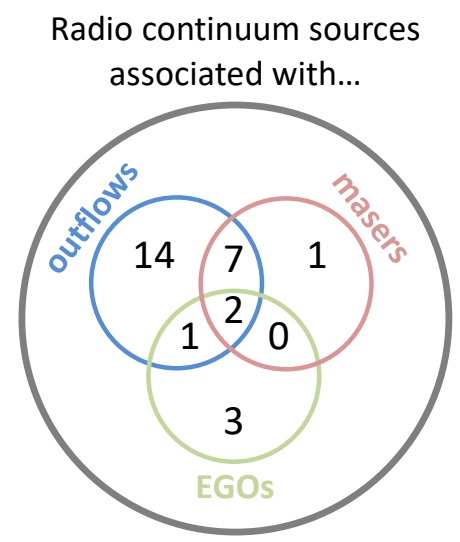

Association with outflows:

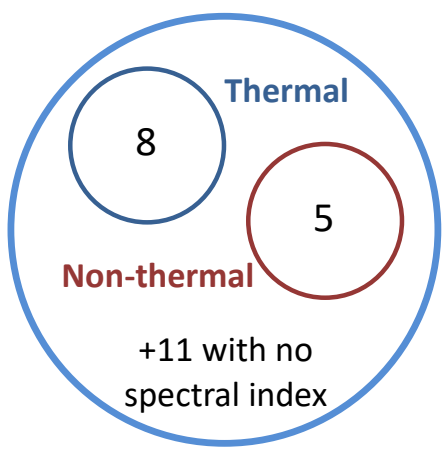

Association with EGOs:

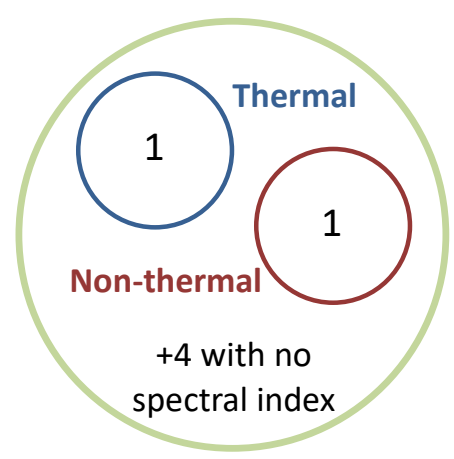

Association with masers:

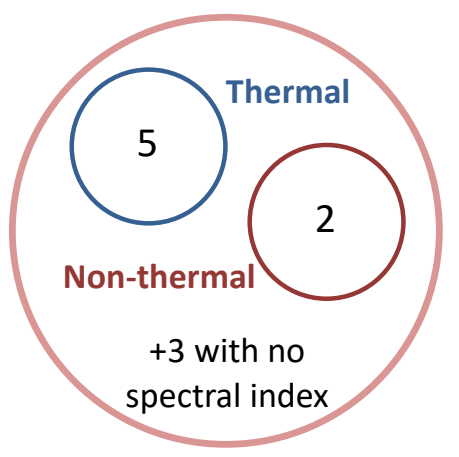

Fig. 4. Diagrams summarizing the outflow-activity associations of the radio jet candidates studied in this work. The integer numbers indicate the number radio jet candidates in a specific group (see Table 3). The top diagram summarizes the association of the radio jet candidates with molecular outflows, masers and EGOs (see Sect. 4, for EGOs we only consider association if the source is labeled as ' $\mathrm{Y}$ ' in Table 3). The bottomrow diagrams summarize the results regarding the thermal (spectral index $>-0.1$ ) and non-thermal (spectral index $<-0.1$ ) properties of the radio continuum emission. The number of sources for which we could not derive the spectral index is also indicated.

Table 4. Number of the sources with thermal and non-thermal radio continuum emission associated with different outflow activity signatures.

\begin{tabular}{lcc}
\hline \hline & Non-thermal Sources & Thermal Sources \\
\hline Outflows & $5 / 5(100 \%)$ & $7 / 8(88 \%)$ \\
EGOs & $1 / 5(20 \%)$ & $1 / 8(13 \%)$ \\
Masers (all) & $2 / 5(40 \%)$ & $5 / 8(63 \%)$ \\
$\mathrm{H}_{2} \mathrm{O}$ & $2 / 5(40 \%)$ & $5 / 8(63 \%)$ \\
$\mathrm{CH}_{3} \mathrm{OH}$ & $0 / 5(0 \%)$ & $4 / 8(50 \%)$ \\
\hline
\end{tabular}

of outflow and shock activity. In the following, we build on the properties of the identified radio jet candidates.

\subsubsection{Radio continuum properties}

Reynolds (1986) describe radio jets with a model that assumes a jet of varying temperature, velocity, and ionization fraction. In case of constant temperature, the relations of the flux density $\left(S_{v}\right)$ and source size $\left(\theta_{v}\right)$ with frequency are given by

$S_{v} \propto v^{\alpha}=v^{1.3-0.7 / \epsilon} \quad$ and $\quad \theta_{v} \propto v^{\beta}=v^{-0.7 / \epsilon}$,

where $\epsilon$ depends only on the geometry of the jet and is the powerlaw index that describes how the width of the jet varies with the distance from the central object. In this model, the spectral index $\alpha$ is always smaller than 1.3 and drops to values $<0.6$ for confined jets $(\epsilon<1$; Anglada et al. 1998).
In Table 3, we list the spectral index $(\alpha)$ and the source size index $(\beta)$ for our radio jet candidates. The latter only for the sources detected at both frequencies. Nine of the radio jet candidates associated with outflow/shock activity have spectral indices consistent with thermal emission $(>-0.1)$, with six showing clear positive $(>+0.4)$ spectral indices. These values are consistent with the model of Reynolds (1986) for values of $\epsilon>0.6$. For such geometries of the jet, the source size index $(\beta)$ is expected to be about -1 . The source size indices reported in Table 3 are mainly in the range -0.1 to -1.0 , in agreement with the model of Reynolds (1986) for radio jets.

Despite most of our radio jet candidates have spectral indices consistent with thermal emission (64\% of the sample, see Table 3), we find some sources (accounting for $36 \%$ of the sample, five sources ${ }^{5}$ ) that show negative spectral indices. This finding is in agreement with some recent works. For example, Moscadelli et al. (2016) find about 20\% of their sample of 15 radio continuum sources to be associated with non-thermal emission. The presence of non-thermal emission is explained in terms of synchrotron emission from relativistic electrons accelerated in strong shocks within the jets, and a number of cases have been studied in more detail (e.g., Carrasco-González et al. 2010; Sanna et al. 2019). Further detailed observations towards these new four non-thermal radio jet candidates, can provide further

\footnotetext{
5 As discussed in Sect. 4.5.3, four of these five sources are most likely $\mathrm{H}$ II regions. This would reduce the number of non-thermal radio jets to only one out of 14 ( $7 \%$ of our sample).
} 
constraints to understand the characteristics of this kind of objects.

We have searched for possible relations between the presence of thermal and non-thermal radio jets and different outflow/shock activity signposts (i.e., outflows, masers and EGOs). We summarize our findings in the bottom panels of Fig. 4). We do not find a preferred relation between thermal and nonthermal radio jets with the outflow activity signposts, since we find similar percentages (see Table 4) for the association with outflows (88\% and 100\%, respectively), EGOs (13\% and 20\%), and masers (55\% and 40\%). The low number of objects included in our analysis prevents us from deriving further conclusions, and we indicate that a larger sample of radio jets needs to be studied to better understand the properties and differences between thermal and non-thermal radio jets. It is also worth noting that all the four objects associated with both $\mathrm{CH}_{3} \mathrm{OH}$ and $\mathrm{H}_{2} \mathrm{O}$ masers are thermal radio jet candidates (see Table 3 ), while only one of the three objects associated with only $\mathrm{H}_{2} \mathrm{O}$ masers shows thermal emission. This might suggest that radio jets associated with $\mathrm{CH}_{3} \mathrm{OH}$ masers tend to have positive spectral indices (i.e., thermal emission), while radio jets associated with only $\mathrm{H}_{2} \mathrm{O}$ masers might preferentially have negative spectral indices (i.e., non-thermal emission). However, the low number of sources studied in our sample prevents from deriving further conclusions. One should also note that the different levels of association of the radio continuum sources with maser emission may be affected by the variability of masers (Felli et al. 2007; Sugiyama et al. 2017; Ashimbaeva et al. 2017). Moreover, we can not discard that the poor spectral resolution of our observations, which may smear out the intensity of the maser lines making some of them undetectable with our sensitivity limits, may also affect our detectability limits. Despite these limitations, our results are in agreement with the $6.7 \mathrm{GHz} \mathrm{CH}_{3} \mathrm{OH}$ masers tracing the actual location of the newly-born YSOs usually associated with thermal winds/jets, while $\mathrm{H}_{2} \mathrm{O}$ masers may be originated in strong shocks where non-thermal synchrotron emission can be relevant (see e.g., Moscadelli et al. 2013, 2016).

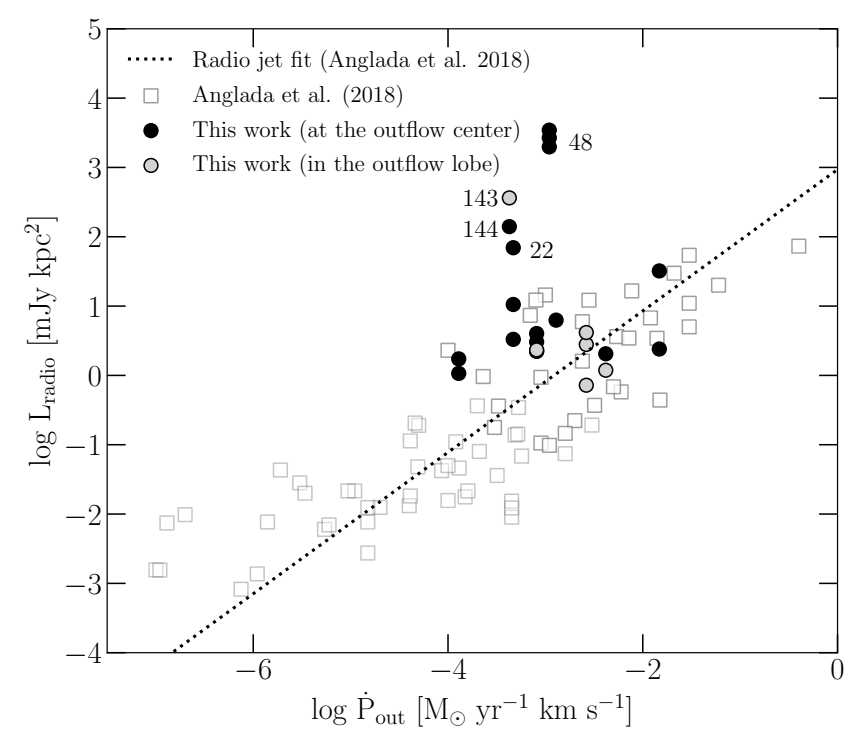

Fig. 5. Relation between radio luminosity $\left(L_{\text {radio }}\right)$ and outflow momentum rate $\left(\dot{P}_{\text {out }}\right)$. Open boxes show data from (Anglada et al. 2018, see also Anglada et al. 1992). The dashed line is a least-squares fit to the radio jets reported by Anglada et al. (2018), corresponding to $\left[L_{\text {radio }} / \mathrm{mJy} \mathrm{kpc}^{2}\right]=10^{+2.97}\left[\dot{P} / M_{\odot} \mathrm{yr}^{-1} \mathrm{~km} \mathrm{~s}^{-1}\right]^{+1.02}$ (see their Eq. 31).

\subsubsection{Jet-outflow connection}

It has been found that the radio luminosity $\left(L_{\mathrm{rad}}=S_{\nu} d^{2}\right)$ of thermal radio jets is correlated with the energetics of the associated molecular outflows. The relationship between radio luminosity and momentum rate in the outflow $\left(\dot{P}_{\text {out }}\right)$ is empirically derived by Anglada et al. (1992, see also Anglada et al. 2018). In Fig. 5, we compare our radio jet candidates (see Table 3) with the radio jets studied by Anglada et al. (2018). As reported by Anglada et al. (2018), there is a tight correlation between the radio luminosity of the jet and the outflow momentum rate. This relationship is interpreted as proof that shocks are the ionization mechanism of radio jets (see e.g., Rodríguez et al. 2008; Anglada et al. 2018). Most of the radio jet candidates investigated in this work, with the exception of only a few sources, follow this relationship, suggesting a radio jet origin for the detected radio continuum emission. The exceptions are mainly the sources \#48a, \#48b, \#48c, \#143 and \#144, which have a much larger radio luminosity compared to the associated outflow momentum rate. This excess suggests that another mechanism could be responsible for a large fraction of the observed radio continuum emission. Based on the location of these sources in the diagram shown in Fig. 3, these sources may correspond to more evolved and extended $\mathrm{H}$ II regions, instead of radio jets, thus explaining the discrepancy between the observed radio luminosity and outflow momentum rate. In this case, we could be facing two possible scenarios. The first is that the sources are indeed radio jets transitioning into a more evolved $\mathrm{H}$ II region phase (similar to what has been proposed for G35.20-0.74N, Beltrán et al. 2016). The second scenario is that the radio continuum sources that we are detecting are only associated with an $\mathrm{H}$ II region, and the spatial coincidence with the molecular outflow emission is due to the presence of another (lower-mass) object powering the outflow but with non-detectable radio continuum emission in our maps. Higher angular resolution observations of the molecular outflow can better establish the location of the powering source and its association with the detected radio continuum sources.

Following Eq. 8 of Anglada et al. (2018, see also Reynolds $1986)$, we estimate the ionized mass-loss rate $\left(\dot{M}_{\text {ion }}\right)$ of our radio jet candidates as

$$
\begin{aligned}
\left(\frac{\dot{M}_{\text {ion }}}{10^{-6} M_{\odot} \mathrm{yr}^{-1}}\right)= & 0.108\left(\frac{d}{\mathrm{kpc}}\right)^{1.5}\left[\frac{(2-\alpha)(0.1+\alpha)}{1.3-\alpha}\right]^{0.75} \\
& \times\left(\frac{T}{10^{4} \mathrm{~K}}\right)^{-0.075}\left[\left(\frac{S_{v}}{\mathrm{mJy}}\right)\left(\frac{v}{10 \mathrm{GHz}}\right)^{-\alpha}\right]^{0.75} \\
& \times\left(\frac{V_{\text {jet }}}{200 \mathrm{~km} \mathrm{~s}^{-1}}\right)\left(\frac{v_{m}}{10 \mathrm{GHz}}\right)^{0.75 \alpha-0.45} \\
& \times\left(\frac{\theta_{0}}{\mathrm{rad}}\right)^{0.75}(\sin i)^{-0.25},
\end{aligned}
$$

where $\alpha$ is the spectral index and $S_{v}$ is the radio continuum flux, both listed in Table 3 , and $d$ is the distance to the source. The opening angle of the jet $\theta_{0}$ can be approximated as $2 \arctan \left(\theta_{\text {min }} / \theta_{\text {maj }}\right)$ (Beltrán et al. 2001; Anglada et al. 2018). We assume a value of 0.5 for the ratio of the minor and major axis of the jet. We also assume that the velocity of the jet $\left(V_{\text {jet }}\right)$ is $500 \mathrm{~km} \mathrm{~s}^{-1}$ and that it lies in the plane of the sky (i.e., $\sin i=1$ ). For a random orientation of the jet on the celestial plane, the value of $\sin i$ is on average $\pi / 4$ (e.g., Beltrán et al. 2001). Usually, a value of $T=10^{4} \mathrm{~K}$ is adopted for ionized gas. For the turnover frequency $v_{m}$, we assume a value of $40 \mathrm{GHz}$ (see discussion in Anglada et al. 2018). In Fig. 6, we show the relationship between the mass loss rates of the ionized and the molecular 


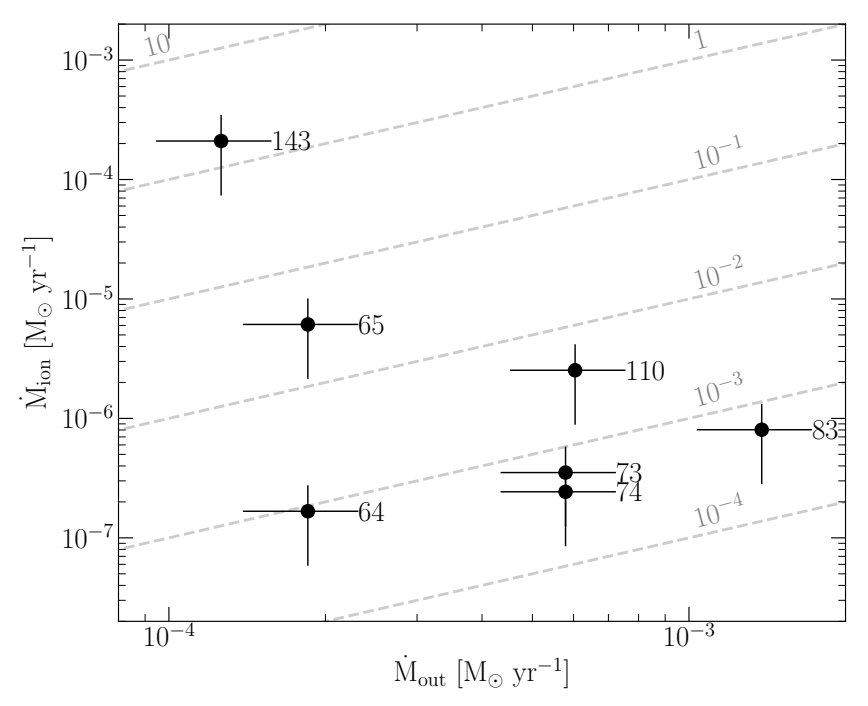

Fig. 6. Relation between ionized $\left(\dot{M}_{\text {ion }}\right)$ and molecular outflow $\left(\dot{M}_{\text {out }}\right)$ mass loss rates for the radio jet candidates listed in Table 3 . The ionized mass loss rate is derived for the thermal radio jets using Eq. 3, while the molecular outflow mass loss rate is provided in López-Sepulcre et al. (2010) and Sánchez-Monge et al. (2013d). The dashed lines indicate different ionization levels given by the ratio $\dot{M}_{\text {ion }}$ to $\dot{M}_{\text {out }}$.

outflow for the thermal radio jet candidates listed in Table 3 and associated with the molecular outflows. Major uncertainties in the determination of $\dot{M}_{\text {ion }}$ may arise from parameters such as the jet velocity, the turnover frequency or the aspect ratio of the jet, as they cannot be determined from our observational data. However, their effects are almost negligible and variations within reasonable ranges result in variations of the ionized mass loss rate of less than a factor of 10 . Our derived $\dot{M}_{\text {ion }}$ are mainly in the range of $10^{-7}$ to $10^{-5} M_{\odot} \mathrm{yr}^{-1}$, consistent with the values reported for low-mass radio jets $\left(\approx 10^{-10} M_{\odot} \mathrm{yr}^{-1}\right)$ and high-mass radio jets $\left(\approx 10^{-5} M_{\odot} \mathrm{yr}^{-1}\right.$, see Rodriguez et al. 1994; Beltrán et al. 2001; Guzmán et al. 2010, 2012). The dashed lines in Fig. 6 indicate different degrees of ionization for the mass loss rate. Most of our radio jet candidates, with the exception of source \#143, which is probably associated with an already developed $\mathrm{H}$ II region (see Fig. 5 and discussion above), have ionization levels of $\dot{M}_{\text {ion }}=$ $10^{-3} \times \dot{M}_{\text {out }}$. These values are about one to two orders of magnitude smaller than those reported in previous studies (see e.g., Rodriguez et al. 1990; Hartigan et al. 1994; Bacciotti et al. 1995; Anglada et al. 2018). This difference may be due to uncertainties in the assumed parameters of Eq. 3, as well as to the fact that our molecular outflow emission is studied with a single-dish (sensitive to all scale structures), while the radio jet observations were carried out with a large interferometric configuration likely resolving out part of the radio jet emission.

\subsubsection{Best radio jet candidates}

In previous sections, we have analyzed the properties of the 146 detected radio continuum sources and built a sample of possible radio jet candidates based on their association with outflow activity: molecular outflows, EGOs, and masers (see Table 3). In Sect. 4.5.1 and 4.5.2, we have investigated in more detail the possible nature of the radio continuum emission and its relation to the outflow activity tracers, in particular, the outflow momentum rate. The results presented in Figs. 3 and 5 allow us to identify sources with properties that differ from those expected from ra- dio jets, therefore suggesting that these sources may actually not be radio jets. From this analysis and the individual description of selected sources (see Appendix A), we discuss in this section which objects are most likely to be radio jets.

Out of the 28 sources listed in Table 3 , five of them have radio luminosities similar to those expected for $\mathrm{H}$ II regions: \#48a, $\# 48$ b, \#48c, \#143 and \#144 (see Fig. 3). In addition, all of these sources, with the exception of \#143, have negative spectral indices. These negative values could be due to the sources being slightly extended (as expected for $\mathrm{H}$ II regions) and partially filtered out in the $\mathrm{K}$ band images. Moreover, these five sources also exhibit large radio luminosities compared to their associated outflow momentum rates (see Fig. 5), which supports the interpretation that there may be an excess of radio continuum emission not necessarily associated with a radio jet, but with an $\mathrm{H}$ II region. In the absence of further evidence, we are not in a position to interpret further and we can not consider these sources to be among the best radio jet candidates. Further observations, sensitive to extended emission, can provide the necessary information to better characterize these sources in terms of their spatial extend and the nature of the emission. It is also worth noting that sources with negative spectral indices could correspond to background sources with synchrotron radiation since we expect about 11 objects in our sample to have this possible origin (see Sect. 3.1). Source \#22 also shows an excess of radio-continuum emission compared to its outflow momentum rate, which suggests that this is also a dubious radio jet candidate. From the individual source descriptions presented in Appendix A, sources \#42 and \#137 seem to be radio continuum sources with most of their emission dominated by cometary/ultracompact $\mathrm{H}$ II regions, which makes it difficult to identify a radio jet in our data.

Out of the remaining sources listed in Table 3, we can identify seven of them having a high probability to be radio jets. These are sources \#2, \#14, \#22, \#64, \#74, \#83 and \#110, which are associated with additional outflow/shock activity such as masers and EGOs. One should note that source \#74 is adjacent to two $\mathrm{H}_{2} \mathrm{O}$ maser features, which are only $2^{\prime \prime}$ away and coincident with extended $4.5 \mu \mathrm{m}$ emission (see Fig. A.7). The remaining sources do still classify as radio jet candidates, since we do not have clear evidence against that. Some of them are located at the center of molecular outflows (e.g., \#95) but are not associated with additional outflow/shock signposts. This could be related to the variability of $\mathrm{H}_{2} \mathrm{O}$ masers (see Sect. 4.5.1). Others are located within molecular outflow lobes (e.g., \#12, \#63, \#64, \#65, $\# 73$, and \#144), and for which higher angular resolution observations of outflow tracers are necessary to confirm if they are powering some of the molecular outflows. Other sources, despite not being associated with molecular outflows, show other shock activity signposts such as the presence of EGOs (e.g., \#119, \#136, \#139). Further observational constrains are therefore needed to fully confirm or discard these objects as radio jets. The results acquired so far, allow us to classify them as radio jet candidates.

\section{Implications for high-mass star formation}

Recently, Rosero et al. (2019) studied the properties of 70 radio continuum sources associated with the earliest stages of highmass star formation. They find that $\approx 30-50 \%$ of their sample are ionized jets. This fraction is in agreement with our findings. Out of the 146 radio continuum sources detected in our study, we identify 28 possible radio jets (i.e., about $19 \%$ of our sample). However, if we focus on the sources for which we have more accurate information (i.e., sources classified as $\mathrm{iC} / \mathrm{iK}$ in Table B.1, see also Sect. 3.1 ), we have 24 out of 40 sources being potential 
radio jets. Therefore, the percentage of radio continuum sources being radio jets increases up $60 \%$. This suggests that about half of the radio continuum sources found in star-forming regions at early evolutionary stages may indeed be radio jets powered by young stars. The remaining $\approx 50 \%$ of objects could still be radio jets for which we have not yet identified shock activity signposts, or they could represent extremely compact $\mathrm{H}$ II regions in their early stages of development. These objects could be powered by early B-type stars and not necessarily by the most massive stars, and could be an intermediate stage between radio jets and already developed H II regions (see e.g., Beltrán et al. 2016; Rivera-Soto et al. 2020).

López-Sepulcre et al. (2010) classified the regions studied in our work as infrared dark (IRDC, infrared dark cloud) and infrared bright (HMSFR, high-mass star-forming region) based on their detectable infrared emission. Our sample, therefore, consists of two sub-classes: IRDC ( 8 regions) and HMSFR (10 regions; see Table 1). We assume that these two types belong to different evolutionary phases of massive star formation, with the IRDC regions being less evolved than the HMSRF regions. Considering the 40 sources located within the primary beam of our images (i.e., sources classified as $\mathrm{iC} / \mathrm{iK}$ ), we find $\approx 2.8$ radio continuum sources per HMSFR region, and $\approx 1.8$ radio continuum sources per IRDC region. This suggests that it is more probable to detect compact radio continuum emission in more evolved regions. Regarding the presence of radio jets in these two evolutionary stages, we find 21 out of the 28 radio jet candidates listed in Table 3 in HMSFR regions (corresponding to 75\%), while we only find 7 (corresponding to $25 \%$ ) in the less evolved IRDC regions. If we consider only the best radio jet candidates (see Sect. 4.5.3), we find five radio jets (\#2, \#14, \#22, \#64 and \#83; corresponding to $71 \%$ ) in HMSRF regions and two radio jets (\#74 and \#110; corresponding to 29\%) in IRDC regions. This shows a preference of radio jets to be found in more evolved clouds. Complementary to this, we can determine the fraction of IRDC and HMSFR regions that harbor radio jets. Out of the 8 IRDC regions studied in this work, only 2 (corresponding to $25 \%$ ) harbor one of the best radio jet candidates. This increases up to $50 \%$ (5 out of 10) for the HMSFR regions. Therefore, the frequency of radio jets in IRDC regions is lower than in HMSFR regions. One possible explanation is that the jets may become larger and brighter with time. Our limited data do not show that IRDC jets are smaller or fainter than HMSFR jets, but future work on larger source samples may provide further insight.

\section{Summary}

In this work, we have used of the VLA in two different bands ( $\mathrm{C}$ and $\mathrm{K}$ band, corresponding to 6 and $1.3 \mathrm{~cm}$ wavelengths) to search for radio jets powered by high-mass YSOs. We have studied a sample of 18 high-mass star-forming regions with signposts of $\mathrm{SiO}$ and $\mathrm{HCO}^{+}$outflow activity. In the following we summarize our main results.

- We have identified 146 radio continuum sources in the 18 high-mass star forming regions, with 40 of the radio continuum sources located within the primary beams of our images (i.e., labeled as $\mathrm{iC} / \mathrm{iK}$ and with reliable flux measurements). Out of these sources, 131 ( $27 \mathrm{iC} / \mathrm{iKs})$ are only detected in the $\mathrm{C}$ band, 4 ( $3 \mathrm{iC} / \mathrm{iKs}$ ) are only detected in the $\mathrm{K}$ band, and $11(10 \mathrm{iC} / \mathrm{iKs})$ are detected in both bands. This different detection level is likely due to different factors: (i) four regions were not observed in the $\mathrm{K}$ band, (ii) the $\mathrm{C}$ band images have a larger field of view, and (iii) the $\mathrm{K}$ band images are affected by a larger interferometric spatial filtering. In addition to the continuum emission, we have detected 23 maser features in

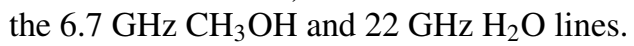

- Out of the 146 continuum sources, only 40 sources are located within the field of view of both images allowing for an accurate characterization of their radio properties. For these sources we have derived the spectral index, which we find to be consistent with thermal emission (i.e., in the range -0.1 to +2.0$)$ for most of the objects $(73 \%)$.

- We have investigated the nature of the radio continuum emission by comparing the radio luminosity to the bolometric luminosity. We find that most sources with positive spectral indices (i.e., thermal emission) follow the trend expected for radio jets, while sources with large negative spectral indices seem to follow the relation expected for $\mathrm{H}_{\text {II }}$ regions. These large negative spectral indices are likely due to the emission in the $\mathrm{K}$ band images being partially filtered out.

- Based on the association of the radio continuum sources with shock activity signposts (i.e., association with molecular outflows, EGOs or masers), we have compiled a list of 28 radio jet candidates. This corresponds to $\approx 60 \%$ of the radio continuum sources located within the field of view of both VLA images. Out of these sample of radio jet candidates, we have identified 7 objects (\#2, \#14, \#22, \#64, \#74, \#83, and \#110) as the most probable radio jets. The remaining 21 require additional observations, either at different radio frequency bands or of molecular outflow tracers at higher resolution, to confirm or discard them as radio jets.

- We find about 7-36\% of the possible radio jet candidates to show non-thermal radio continuum emission. This is consistent with previous studies reporting $\approx 20 \%$ of non-thermal radio jets. We do not find a clear association of the non-thermal emission with the presence of outflows, EGOs or masers. However, and despite the low statistics, we find that radio jet candidates associated with $\mathrm{CH}_{3} \mathrm{OH}$ masers have thermal emission, while those radio jet candidates associated with only $\mathrm{H}_{2} \mathrm{O}$ masers tend to have non-thermal emission. This is in agreement with the $6.7 \mathrm{GHz} \mathrm{CH}_{3} \mathrm{OH}$ masers tracing the actual location of newly-born YSOs powering thermal winds and jets, while the $\mathrm{H}_{2} \mathrm{O}$ masers may be originated in strong shocks where non-thermal emission becomes relevant.

- As previously found in other works, we find a correlation between the radio luminosity of our radio jet candidates and their associated outflow momentum rates. We derive an ionized mass loss rate in the range $10^{-7}$ to $10^{-5} M_{\odot} \mathrm{yr}^{-1}$, which results in ionization levels of $\dot{M}_{\text {ion }}=10^{-3} \dot{M}_{\text {out }}$ (i.e., $\approx 0.1 \%$ of the outflow mass being ionized).

- The 18 high-mass star-forming regions studied in this work are classified in two different evolutionary stages: 8 less evolved IRDC and 10 more evolved HMSFR. We find more radio continuum sources $(\approx 2.8$ sources per region) in the more evolved HMSFR compared to the IRDC $(\approx 1.8)$. Regarding radio jets, we find about $71 \%$ of the radio jet candidates to be located in HMSFR regions, and only $29 \%$ in IRDC regions. Complemenary to this, $25 \%$ of the IRDC regions harbor one of the most probable radio jet candidates, while this percentage increases up to $50 \%$ for the HMSFR regions. This suggests that the frequency of radio jets in the less evolved IRDC regions is lower compared to the more evolved HMSFR regions.

Acknowledgements. The authors thank the referee for his/her review and greatly appreciate the comments and suggestions that have contributed significantly to improve the quality of the publication. ÜK also thanks Jonathan Tan for useful discussions. This work has been partially supported by the Scientific and Technological Research Council of Turkey (TÜBİTAK), project number: 116 F003. 
Part of this work was supported by the Research Fund of Istanbul University, project number: 44659 . ASM research is carried out within the Collaborative Research Centre 956, sub-project A6, funded by the Deutsche Forschungsgemeinschaft (DFG; project ID 184018867). ÜK would like to thank William Pearson for checking the language of the paper and Kyle Oman for helping with Python issues.

\section{References}

Andre, P., Ward-Thompson, D., \& Barsony, M. 2000, in Protostars and Planets IV, ed. V. Mannings, A. P. Boss, \& S. S. Russell, 59

Anglada, G. 1995, in Revista Mexicana de Astronomia y Astrofisica Conference Series, Vol. 1, Revista Mexicana de Astronomia y Astrofisica Conference Series, ed. S. Lizano \& J. M. Torrelles, 67

Anglada, G. 1996, in Astronomical Society of the Pacific Conference Series, Vol. 93, Radio Emission from the Stars and the Sun, ed. A. R. Taylor \& J. M. Paredes, 3-14

Anglada, G., Rodriguez, L. F., Canto, J., Estalella, R., \& Torrelles, J. M. 1992, ApJ, 395, 494

Anglada, G., Rodríguez, L. F., \& Carrasco-González, C. 2018, A\&A Rev., 26, 3 Anglada, G., Villuendas, E., Estalella, R., et al. 1998, AJ, 116, 2953

Arce, H. G., Shepherd, D., Gueth, F., et al. 2007, in Protostars and Planets V, ed. B. Reipurth, D. Jewitt, \& K. Keil, 245

Ashimbaeva, N. T., Colom, P., Krasnov, V. V., et al. 2017, Astronomy Reports, 61,16

Azatyan, N. M., Nikoghosyan, E. H., \& Khachatryan, K. G. 2016, Astrophysics, 59,339

Bacciotti, F., Chiuderi, C., \& Oliva, E. 1995, A\&A, 296, 185

Bachiller, R. 1996, ARA\&A, 34, 111

Bally, J. 2016, ARA\&A, 54, 491

Battersby, C., Bally, J., Jackson, J. M., et al. 2010, ApJ, 721, 222

Beltrán, M. T., Cesaroni, R., Moscadelli, L., et al. 2016, A\&A, 593, A49

Beltrán, M. T., Estalella, R., Anglada, G., Rodríguez, L. F., \& Torrelles, J. M. 2001, AJ, 121, 1556

Beltrán, M. T., Sánchez-Monge, Á., Cesaroni, R., et al. 2014, A\&A, 571, A52

Beuther, H., Schilke, P., Sridharan, T. K., et al. 2002a, A\&A, 383, 892

Beuther, H., Walsh, A., Schilke, P., et al. 2002b, A\&A, 390, 289

Breen, S. L., Ellingsen, S. P., Contreras, Y., et al. 2013, MNRAS, 435, 524

Carrasco-González, C., Rodríguez, L. F., Anglada, G., et al. 2010, Science, 330, 1209

Carrasco-González, C., Torrelles, J. M., Cantó, J., et al. 2015, Science, 348, 114 Caswell, J. L., Fuller, G. A., Green, J. A., et al. 2010, MNRAS, 404, 1029

Caswell, J. L. \& Green, J. A. 2011, MNRAS, 411, 2059

Cesaroni, R., Sánchez-Monge, Á., Beltrán, M. T., et al. 2017, A\&A, 602, A59

Cesaroni, R., Sánchez-Monge, Á., Beltrán, M. T., et al. 2016, A\&A, 588, L5

Chini, R., Kruegel, E., \& Wargau, W. 1987, A\&A, 181, 378

Condon, J. J., Cotton, W. D., Greisen, E. W., et al. 1998, AJ, 115, 1693

Curiel, S., Canto, J., \& Rodriguez, L. F. 1987, Rev. Mexicana Astron. Astrofis., 14,595

Curiel, S., Rodríguez, L. F., Cantó, J., et al. 1989, Astrophysical Letters and Communications, 27, 299

Cutri, R. M. \& et al. 2012, VizieR Online Data Catalog, II/311

Cyganowski, C. J., Brogan, C. L., Hunter, T. R., \& Churchwell, E. 2009, ApJ, 702,1615

Cyganowski, C. J., Brogan, C. L., Hunter, T. R., \& Churchwell, E. 2011, ApJ, 743,56

Cyganowski, C. J., Brogan, C. L., Hunter, T. R., et al. 2012, ApJ, 760, L20

Cyganowski, C. J., Koda, J., Rosolowsky, E., et al. 2013, ApJ, 764, 61

Cyganowski, C. J., Whitney, B. A., Holden, E., et al. 2008, AJ, 136, 2391

De Buizer, J. M., Radomski, J. T., Telesco, C. M., \& Piña, R. K. 2005, ApJS, 156,179

de Villiers, H. M., Chrysostomou, A., Thompson, M. A., et al. 2015, MNRAS, 449, 119

Elitzur, M. 1982, Reviews of Modern Physics, 54, 1225

Felli, M., Brand, J., Cesaroni, R., et al. 2007, A\&A, 476, 373

Froebrich, D., Makin, S. V., Davis, C. J., et al. 2015, MNRAS, 454, 2586

Gasiprong, N., Cohen, R. J., \& Hutawarakorn, B. 2002, MNRAS, 336, 47

Gaume, R. A., Fey, A. L., \& Claussen, M. J. 1994, ApJ, 432, 648

Ginsburg, A., Glenn, J., Rosolowsky, E., et al. 2013, ApJS, 208, 14

Gordon, M. A. \& Sorochenko, R. L. 2002, Radio Recombination Lines. Their Physics and Astronomical Applications, Vol. 282

Guzmán, A. E., Garay, G., \& Brooks, K. J. 2010, ApJ, 725, 734

Guzmán, A. E., Garay, G., Brooks, K. J., \& Voronkov, M. A. 2012, ApJ, 753, 51

Hartigan, P., Morse, J. A., \& Raymond, J. 1994, ApJ, 436, 125

Hatchell, J., Fuller, G. A., \& Millar, T. J. 2001, A\&A, 372, 281

Helfand, D. J., Becker, R. H., White, R. L., Fallon, A., \& Tuttle, S. 2006, AJ, 131,2525

Hoare, M. G., Purcell, C. R., Churchwell, E. B., et al. 2012, PASP, 124, 939
Johnston, K. G., Robitaille, T. P., Beuther, H., et al. 2015, ApJ, 813, L19

Kalenskii, S. V. \& Kurtz, S. 2016, Astronomy Reports, 60, 702

Kuchar, T. A. \& Clark, F. O. 1997, ApJ, 488, 224

Kurtz, S. 2005, in IAU Symposium, Vol. 227, Massive Star Birth: A Crossroads of Astrophysics, ed. R. Cesaroni, M. Felli, E. Churchwell, \& M. Walmsley, 111-119

Kurtz, S., Churchwell, E., \& Wood, D. O. S. 1994, ApJS, 91, 659

Larson, R. B. 1969, MNRAS, 145, 271

Lekht, E. E. 2000, A\&AS, 141, 185

Leto, P., Umana, G., Trigilio, C., et al. 2009, A\&A, 507, 1467

Liu, T., Wu, Y., \& Zhang, H. 2013, ApJ, 776, 29

Lockman, F. J. 1989, ApJS, 71, 469

López-Sepulcre, A., Cesaroni, R., \& Walmsley, C. M. 2010, A\&A, 517, A66

López-Sepulcre, A., Codella, C., Cesaroni, R., Marcelino, N., \& Walmsley, C. M. 2009, A\&A, 499, 811

López-Sepulcre, A., Walmsley, C. M., Cesaroni, R., et al. 2011, A\&A, 526, L2

Lu, X., Zhang, Q., Liu, H. B., Wang, J., \& Gu, Q. 2014, ApJ, 790, 84

Luhman, K. L. 2012, ARA\&A, 50, 65

Lumsden, S. L., Hoare, M. G., Urquhart, J. S., et al. 2013, ApJS, 208, 11

Marti, J., Rodriguez, L. F., \& Reipurth, B. 1993, ApJ, 416, 208

Maud, L. T., Cesaroni, R., Kumar, M. S. N., et al. 2019, A\&A, 627, L6

McMullin, J. P., Waters, B., Schiebel, D., Young, W., \& Golap, K. 2007, in Astronomical Society of the Pacific Conference Series, Vol. 376, Astronomical Data Analysis Software and Systems XVI, ed. R. A. Shaw, F. Hill, \& D. J. Bell, 127

Moscadelli, L., Cesaroni, R., \& Rioja, M. J. 2005, A\&A, 438, 889

Moscadelli, L., Cesaroni, R., Sánchez-Monge, Á., et al. 2013, A\&A, 558, A145

Moscadelli, L., Sánchez-Monge, Á., Goddi, C., et al. 2016, A\&A, 585, A71

Moscadelli, L., Sanna, A., Goddi, C., et al. 2019, A\&A, 631, A74

Motte, F., Nony, T., Louvet, F., et al. 2018, Nature Astronomy, 2, 478

Palau, A., Sánchez-Monge, Á., Busquet, G., et al. 2010, A\&A, 510, A5

Purcell, C. R., Hoare, M. G., Cotton, W. D., et al. 2013, ApJS, 205, 1

Purser, S. J. D., Lumsden, S. L., Hoare, M. G., \& Cunningham, N. 2018, MNRAS, 475, 2

Rathborne, J. M., Jackson, J. M., \& Simon, R. 2006, ApJ, 641, 389

Rathborne, J. M., Simon, R., \& Jackson, J. M. 2007, ApJ, 662, 1082

Reid, M. J., Argon, A. L., Masson, C. R., Menten, K. M., \& Moran, J. M. 1995, ApJ, 443, 238

Reid, M. J. \& Ho, P. T. P. 1985, ApJ, 288, L17

Reynolds, S. P. 1986, ApJ, 304, 713

Rivera-Soto, R., Galván-Madrid, R., Ginsburg, A., \& Kurtz, S. 2020, ApJ, 899, 94

Rodriguez, L. F. 1995, in Revista Mexicana de Astronomia y Astrofisica Conference Series, Vol. 1, Revista Mexicana de Astronomia y Astrofisica Conference Series, ed. S. Lizano \& J. M. Torrelles, 1

Rodriguez, L. F., Garay, G., Curiel, S., et al. 1994, ApJ, 430, L65

Rodriguez, L. F., Ho, P. T. P., Torrelles, J. M., Curiel, S., \& Canto, J. 1990, ApJ, 352,645

Rodríguez, L. F., Moran, J. M., Franco-Hernández, R., et al. 2008, AJ, 135, 2370

Rosero, V., Hofner, P., Claussen, M., et al. 2016, ApJS, 227, 25

Rosero, V., Hofner, P., Kurtz, S., et al. 2019, ApJ, 880, 99

Roueff, E., Dartois, E., Geballe, T. R., \& Gerin, M. 2006, A\&A, 447, 963

Sánchez-Monge, Á., Beltrán, M. T., Cesaroni, R., et al. 2014, A\&A, 569, A11

Sánchez-Monge, Á., Beltrán, M. T., Cesaroni, R., et al. 2013a, A\&A, 550, A21

Sánchez-Monge, Á., Cesaroni, R., Beltrán, M. T., et al. 2013b, A\&A, 552, L10

Sánchez-Monge, Á., Kurtz, S., Palau, A., et al. 2013c, ApJ, 766, 114

Sánchez-Monge, Á., López-Sepulcre, A., Cesaroni, R., et al. 2013d, A\&A, 557, A94

Sánchez-Monge, Á., Palau, A., Estalella, R., Beltrán, M. T., \& Girart, J. M. 2008, A\&A, 485, 497

Sanna, A., Moscadelli, L., Goddi, C., et al. 2019, A\&A, 623, L3

Sanna, A., Moscadelli, L., Goddi, C., Krishnan, V., \& Massi, F. 2018, A\&A, 619 A 107

Sewiło, M., Churchwell, E., Kurtz, S., Goss, W. M., \& Hofner, P. 2011, ApJS, 194,44

Shepherd, D. S., Nürnberger, D. E. A., \& Bronfman, L. 2004, ApJ, 602, 850

Stecklum, B., Caratti o Garatti, A., Klose, S., \& Wiseman, P. 2017, The Astronomer's Telegram, 10842, 1

Sugiyama, K., Nagase, K., Yonekura, Y., et al. 2017, PASJ, 69, 59

Tan, J. C., Beltrán, M. T., Caselli, P., et al. 2014, in Protostars and Planets VI, ed. H. Beuther, R. S. Klessen, C. P. Dullemond, \& T. Henning, 149

Thompson, R. I. 1984, ApJ, 283, 165

Torrelles, J. M., Ho, P. T. P., Rodriguez, L. F., \& Canto, J. 1985, ApJ, 288, 595

Torrelles, J. M., Patel, N. A., Curiel, S., et al. 2011, MNRAS, 410, 627

Urquhart, J. S., Hoare, M. G., Lumsden, S. L., Oudmaijer, R. D., \& Moore, T. J. T. 2008, in Astronomical Society of the Pacific Conference Series, Vol 387, Massive Star Formation: Observations Confront Theory, ed. H. Beuther, H. Linz, \& T. Henning, 381

Williams, J. P. \& Cieza, L. A. 2011, ARA\&A, 49, 67 


\section{Appendix A: Comments on individual sources}

In the following, we comment on different aspects of selected sources for which additional literature information is available.

\section{IRAS 05358+3543 (\#2 and \#4)}

In region IRAS $05358+3543$, we have identified two radio continuum sources that can be potential radio jets (see Fig. A.1). Sources \#2 and \#4 were observed only in the C band, and therefore we cannot determine a spectral index for these sources. Despite this limitation, both sources are located at the center of the molecular outflow reported by López-Sepulcre et al. (2010). Furthermore, source \#2 is associated with a $6.7 \mathrm{GHz} \mathrm{CH} \mathrm{CH}_{3} \mathrm{OH}$ maser emission, suggesting that this marks the position of a massive YSO. Of the two radio continuum sources, source \#2 is likely the main object powering the molecular outflow for which its centimeter emission traces a radio jet. Further observations at different frequency bands are necessary to better constrain its properties.

\section{G189.78+0.34 (\#12, \#13, \#14, \#15 and \#16)}

In region $\mathrm{G} 189.78+0.34$, we have identified five radio continuum sources (sources \#12 to \#16) associated with the molecular outflow reported by López-Sepulcre et al. (2010). All of them are located at the center of the outflow, with source \#12 slightly offset from the rest (see Fig. A.2). All these sources were observed only in the $\mathrm{C}$ band and no spectral index can be derived. Out of the five sources, source \#14 is associated with $\mathrm{CH}_{3} \mathrm{OH}$ maser emission (see also Caswell et al. 2010) suggesting that this marks the location of a massive YSO. The radio continuum sources are found in an elongated chain extending from the south-east to the north-west. This direction is consistent with the orientation of the molecular outflow (López-Sepulcre et al. 2010). Overall, we consider that source \#14 is the powering source and most likely the main component of the radio jet. The other sources could correspond to different radio continuum knots located along the jet, as seen in other sources (e.g., HH 80-81: Carrasco-González et al. 2010, and G35.20-0.74 N: Beltrán et al. 2016), where the radio continuum knots usually show non-thermal spectral indices. Observations at different frequency bands are necessary to gather information on the spectral index and thermal/nonthermal nature of the different radio continuum sources in the region.

\section{G192.58-0.04 (\#22, \#23 and \#25)}

In region G192.58-0.04, we have identified sources \#22, \#23, and \#25 as potential radio jets (see Fig. A.3). These sources were observed only in the $\mathrm{C}$ band and no spectral index can be derived. Out of the three sources, source \#22 is associated with $\mathrm{CH}_{3} \mathrm{OH}$ maser emission suggesting that this source may mark the location of a massive YSO. The sources are located at the center of the molecular outflow reported by López-Sepulcre et al. (2010). Out of the three sources, we consider that source \#22 is the most probable radio jet. The comparison between the radio continuum luminosity with the outflow momentum rate (see Fig. 5), also confirms this possibility, although there seems to be a slightly excess of radio continuum emission, suggesting that there can be an additional contribution to the radio continuum source (e.g., from an early-stage $\mathrm{H}$ II region).

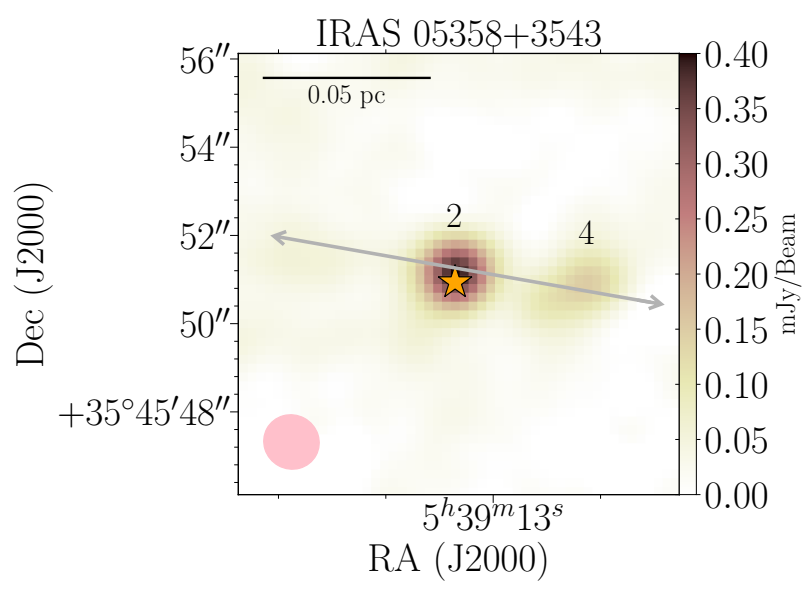

Fig. A.1. VLA C band $(6 \mathrm{~cm})$ continuum emission map of the radio jet candidates \#2 and \#4 located in the region IRAS $05358+3543$. The pink ellipse is the beam size of the $\mathrm{C}$ band. The orange star marks the location of the $\mathrm{CH}_{3} \mathrm{OH}$ maser (see Table 2). The grey double-headed arrow indicates the direction of the outflows.

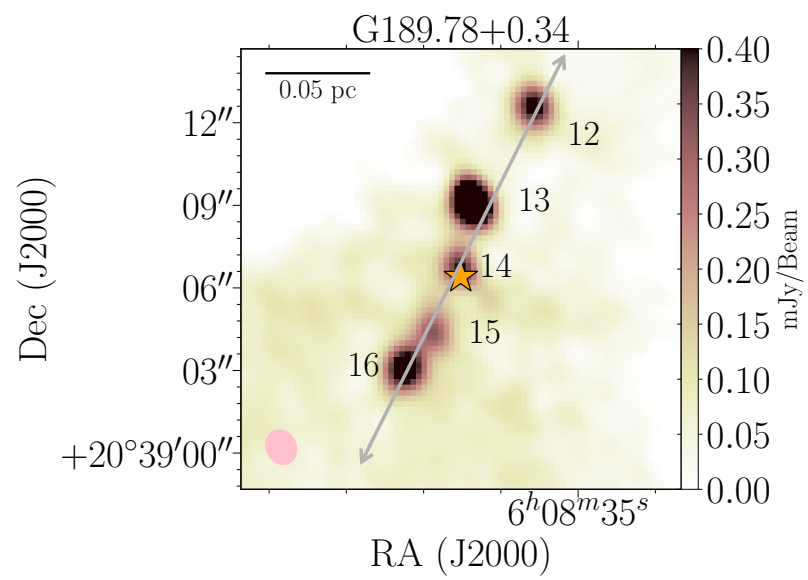

Fig. A.2. VLA C band $(6 \mathrm{~cm})$ continuum emission map of the radio jet candidates \#12, \#13, \#14, \#15 and \#16 located in the region G189.78+0.34. The pink ellipse is the beam size of the $\mathrm{C}$ band. The orange star marks the location of the $\mathrm{CH}_{3} \mathrm{OH}$ maser (see Table 2). The grey double-headed arrow indicates the direction of the outflows.

\section{IRAS 18223-1243 (\#42)}

In the region IRAS 18223-1243, we identified the radio continuum source \#42 (see Fig. A.4) as adjacent to the one reported in Cyganowski et al. (2011) EGO F G18.67+0.03-CM1. This is the only signposts of shock activity, since no molecular outflow or maser emission are found for this object. In addition, Cyganowski et al. (2012) report the existence of a massive protocluster consisting of a hot molecular core and an ultracompact $\mathrm{H}$ II region. Our source seems to be located at the same position of the ultracompact $\mathrm{H}_{\text {II }}$ region, which makes us to doubt if this can be a radio jet candidate.

\section{IRAS 18228-1312 (\#48)}

Radio continuum source \#48 is observed as a group of three compact sources (\#48a, \#48b and \#48c) surrounded by an extended and more diffuse structure. One of these compact sources (\#48a) is clearly associated with $\mathrm{H}_{2} \mathrm{O}$ maser emission (see Fig. A.5). The Spitzer/IRAC $4.5 \mu \mathrm{m}$ emission is extended and 


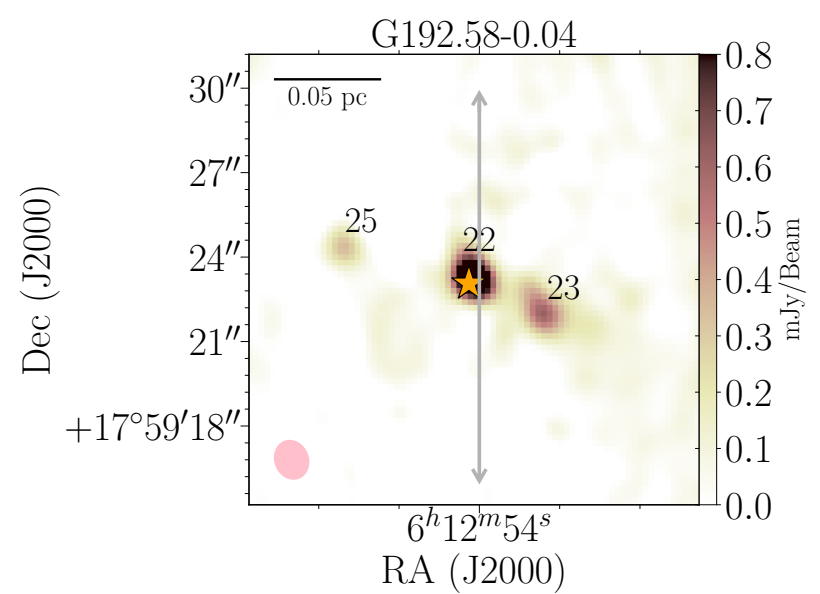

Fig. A.3. VLA C band $(6 \mathrm{~cm})$ continuum emission map of the radio jet candidates \#22, \#23 and \#25 located in the region G192.58-0.04. The pink ellipse is the beam size of the $\mathrm{C}$ band. The orange star marks the location of the $\mathrm{CH}_{3} \mathrm{OH}$ maser (see Table 2). The grey double-headed arrow indicates the direction of the outflows.

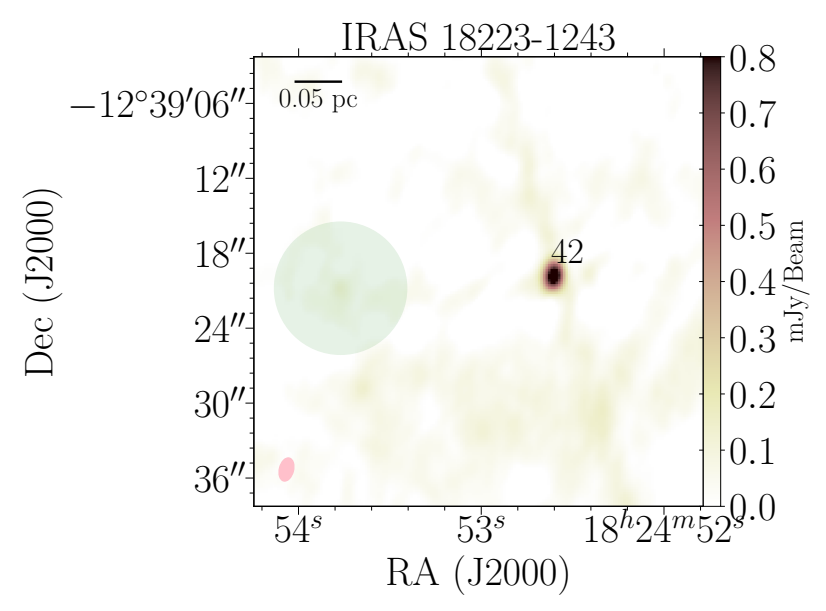

Fig. A.4. VLA C band $(6 \mathrm{~cm})$ continuum emission map of the radio jet candidate \#42 located in the region IRAS 18223-1243. The pink ellipse is the beam size of the $\mathrm{C}$ band. The green circle with a radius of $\sim 5^{\prime \prime}$ marks the EGO F G18.67+0.03-CM1 reported by Cyganowski et al. (2011).

spatially coincident with the radio continuum extended emission. The spectral indices for these three compact sources are in the range -0.6 to -1.5 , most likely due to additional filtering of the emission in the $\mathrm{K}$ band image. Previous studies have classified this extended source as a region containing hypercompact (HC) and ultracompact (UC) H II regions (e.g., Chini et al. 1987; Lockman 1989; Kurtz et al. 1994; Kuchar \& Clark 1997; Leto et al. 2009), which is consistent with our derived radio continuum luminosity (see Figs. 3 and 5). It is worth noting that the the three sources are spatially located at the center of a molecular outflow (e.g., López-Sepulcre et al. 2010). This may suggest that one of the compact sources may be powering the molecular outflow. In this case, this object would be in a evolutionary stage where the radio jet still exists but a young $\mathrm{H}_{\text {II }}$ region has already developed, similar to the high-mass young stellar object G35.20-0.74 N (e.g, Sánchez-Monge et al. 2013b, 2014; Beltrán et al. 2016).

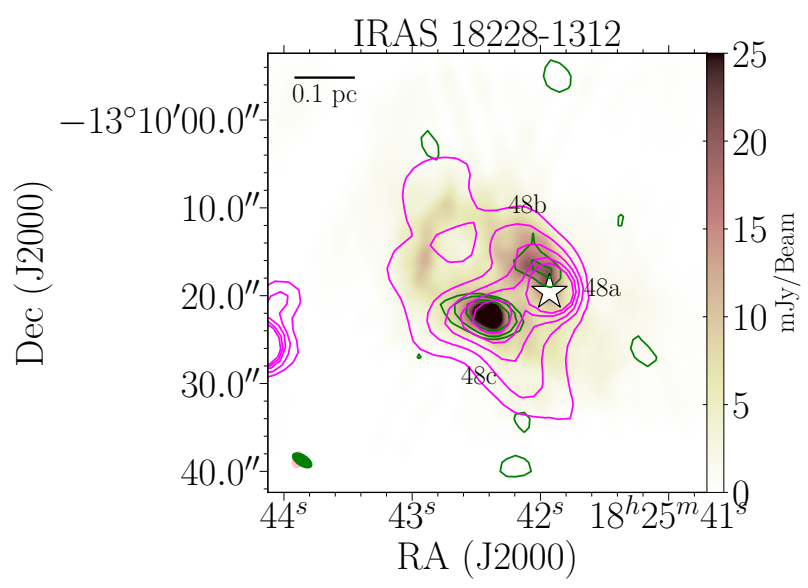

Fig. A.5. VLA C band $(6 \mathrm{~cm})$ continuum emission map of source \#48 located in the region IRAS 18228-1312. Three bright peaks are visible and labelled as \#48a, \#48b and \#48c. The green contour levels of the $\mathrm{K}$ band $(1.3 \mathrm{~cm})$ continuum emission are 3,5 and 9 times $0.7 \mathrm{mJy}_{\text {beam }}{ }^{-1}$. The magenta contours show the Spitzer/GLIMPSE $4.5 \mu \mathrm{m}$ emission. The pink and green ellipses are the beam sizes of the $\mathrm{C}$ and $\mathrm{K}$ bands, respectively. The white star marks the location of the $\mathrm{H}_{2} \mathrm{O}$ maser (see Table 2).

\section{IRAS 18236- 1205 (\#63, \#64 and \#65)}

We have identified nine radio continuum sources in the IRAS 18236-1205 region (also referred to in the literature as G19.36-0.03), three of which have been classified as radio jet candidates: Sources \#63, \#64 and \#65 with spectral indices of $-0.22 \pm 0.07,+0.18 \pm 0.04$ and $+1.08 \pm 0.19$. We have identified four $\mathrm{H}_{2} \mathrm{O}$ maser features near these sources (see Fig. A.6). Two maser features are associated with source \#63, one maser feature is associated with source \#64 (which also spatially coincides with a $\mathrm{CH}_{3} \mathrm{OH}$ maser), and the last maser feature is located in the center of the red-shifted outflow lobe where no radio continuum emission is detected. The molecular outflow in this region has been mapped in the lines $\mathrm{SiO}(2-1)$ and $\mathrm{HCO}^{+}(1-0)$ by (Sánchez-Monge et al. 2013d).

Sources \#63 and \#64 are associated with EGOs (see Cyganowski et al. 2009), indicating the presence of strong shock activity in these two sources. Their location near the center of the molecular outflow together with their association with $\mathrm{H}_{2} \mathrm{O}$ maser emission and EGOs, suggests that these two sources could be candidates for radio jets. The source \#64 is spatially more coincident with the geometric center of the outflow, and its association with $6.7 \mathrm{GHz} \mathrm{CH}_{3} \mathrm{OH}$ maser emission, suggests that a massive YSO exists at this position. This massive YSO could be the driving source of the molecular outflow seen on larger scales. The third candidate (source \#65) is located 28" away from the source \#64 and the center of the outflow, and has been studied by (Cyganowski et al. 2011, G19.36-0.03-CM2). This source is associated with an emission of $4.5 \mu \mathrm{m}$, although it is unclear whether it can be convincingly classified as an EGO (Cyganowski et al. 2009). The positive spectral index indicates the presence of thermal emission, which could come from a radio jet. However, there is no clear evidence of outflow or shock activity. The source \#65 is also located in the vicinity of a dense core (18236-1205 P8) identified by Lu et al. (2014) in the VLA $\mathrm{NH}_{3}$ maps, which supports the interpretation of this source as an embedded young stellar object. Overall, the source \#65 could be a YSO-powered radio jet in the vicinity of the more massive object (sources \#63 and \#64) in IRAS 18236-1205. 

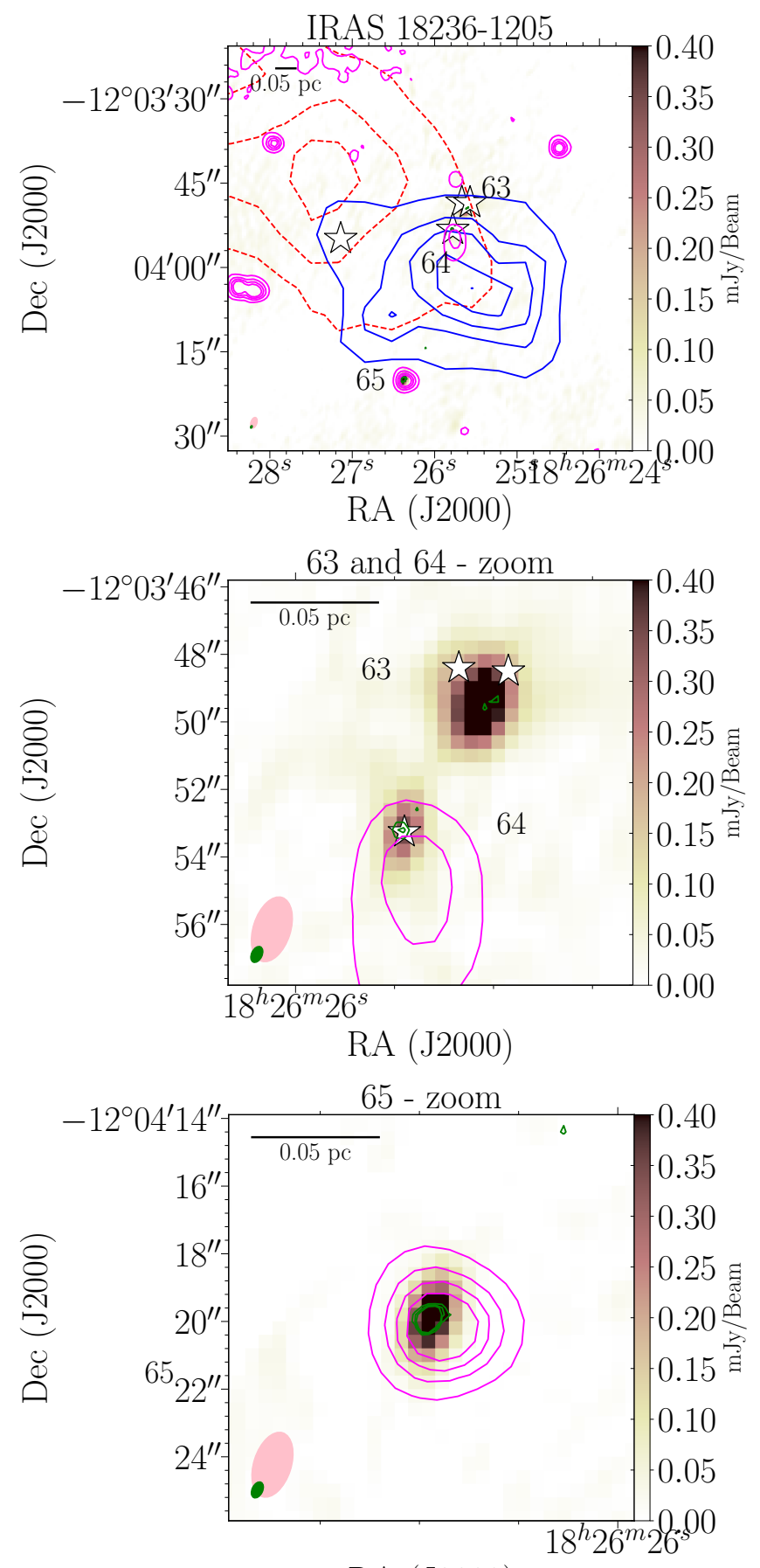

RA (J2000)

Fig. A.6. VLA C band $(6 \mathrm{~cm})$ continuum emission map of the radio jet candidates \#63, \#64 and \#65 located in the region IRAS 18236-1205. A close-up view of the three radio sources is shown in the bottom and right panels. The green contour levels of the $\mathrm{K}$ band $(1.3 \mathrm{~cm})$ continuum emission are 3,5 and 7 times $30 \mu \mathrm{Jy}_{\text {beam }}^{-1}$. The magenta contours show the Spitzer/GLIMPSE $4.5 \mu \mathrm{m}$ emission. The blue- and red-shifted outflow lobed of $\mathrm{SiO}(2-1)$ are shown as blue-solid and red-dashed contours, respectively (see Sánchez-Monge et al. 2013d). The pink and green ellipses are the beam sizes of the $\mathrm{C}$ and $\mathrm{K}$ bands, respectively. The white stars mark the location of the $\mathrm{H}_{2} \mathrm{O}$ masers (see Table 2).

\section{G23.60+0.0M1 (\#73 and \#74)}

The G23.60+0.01M1 star-forming region has been studied in the literature by various authors (e.g., Rathborne et al. 2006; Battersby et al. 2010; Ginsburg et al. 2013), who reported the
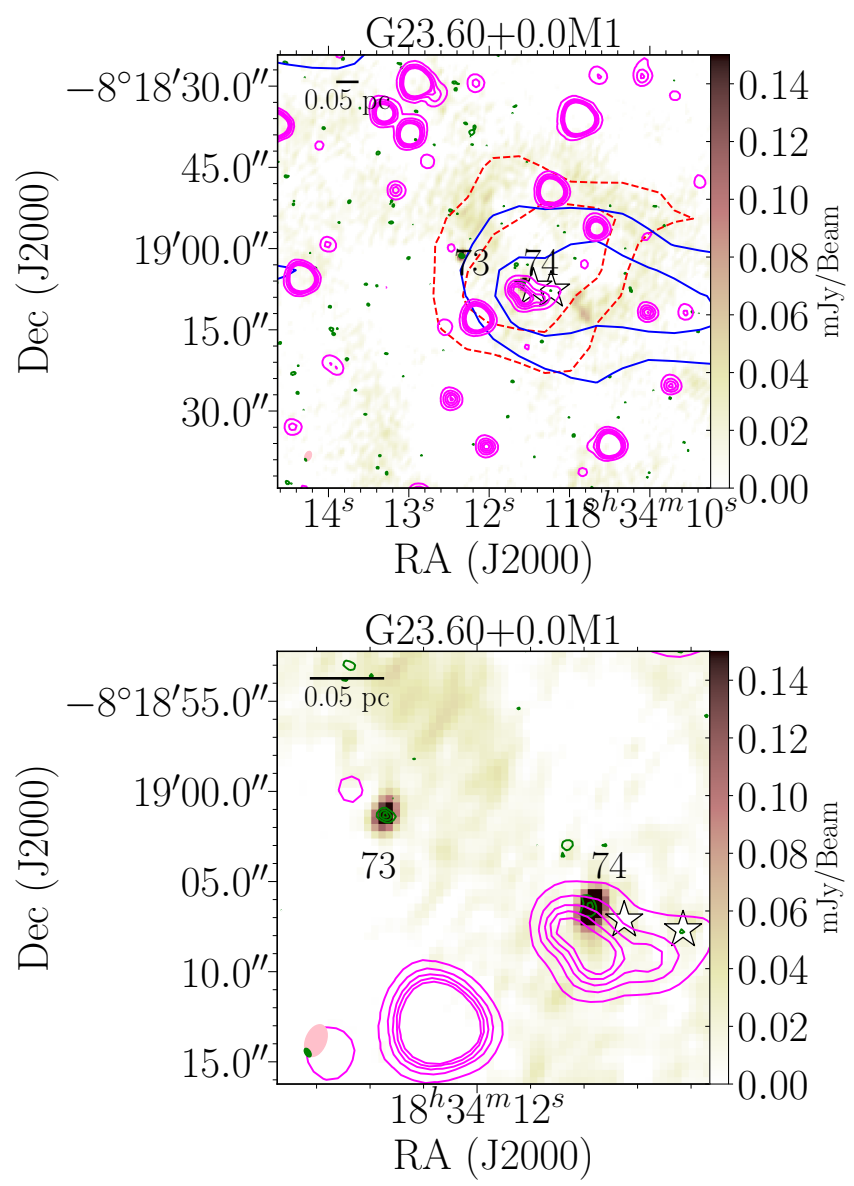

Fig. A.7. VLA C band $(6 \mathrm{~cm})$ continuum emission map of the radio jet candidates \#73 and \#74 located in the region G23.60+0.0M1. A closeup view of the two radio sources is shown in the bottom panel. The green contour levels of the $\mathrm{K}$ band $(1.3 \mathrm{~cm})$ continuum emission are 3 , $5,7,9$ and 11 times $20 \mu \mathrm{Jy}_{\text {beam }}{ }^{-1}$. The magenta contours show the Spitzer/GLIMPSE $4.5 \mu \mathrm{m}$ emission. The blue- and red-shifted outflow lobed of $\mathrm{SiO}(2-1)$ are shown as blue-solid and red-dashed contours, respectively (see Sánchez-Monge et al. 2013d). The pink and green ellipses are the beam sizes of the $\mathrm{C}$ and $\mathrm{K}$ bands, respectively. The white stars mark the location of the $\mathrm{H}_{2} \mathrm{O}$ masers (see Table 2).

presence of two massive dense clumps with masses of $100 M_{\odot}$ and $120 M_{\odot}$. The two candidate radio jets (\#73 and \#74) have positive spectral indices consistent with thermal emission of radio jets. In particular, the source \#74 is located at the center of the molecular outflow reported by Sánchez-Monge et al. (2013d) and is associated with a strong $4.5 \mu \mathrm{m}$ emission (see Fig. A.7). The two $\mathrm{H}_{2} \mathrm{O}$ maser detected in the region are slightly displaced from the radio continuum source but coincide with the extended $4.5 \mu \mathrm{m}$ emission (see Fig. A.7-bottom). The association of molecular outflow emission, bright and extended $4.5 \mu \mathrm{m}$ emission, and close $\mathrm{H}_{2} \mathrm{O}$ maser features favor the interpretation of this source is a good radio jet candidate.

The second radio continuum source $(\# 73)$ is located relatively close to the center of the outflow. However, no maser or EGOs are found in connection with the source. Although we cannot reject this source as a radio jet, we prefer the source \#74 as the main object driving the outflow observed in the region. 


\section{IRAS 18316-0602 (\#83 and \#95)}

We have identified thirteen radio continuum sources in the IRAS 18316-0602 region, two of which have been classified as radio jet candidates: Sources \#83 and \#95. The source \#83 has an almost flat spectral index $(0.08 \pm 0.08)$ and shows a weak extension to the south, which is better resolved in the $\mathrm{K}$ band image. Source \#95 is fainter, appears located about 3 "to the south-east of source \#83, and is visible only in the $\mathrm{K}$ band (some faint emission slightly above the noise level is visible in the $\mathrm{C}$ band image, see Fig. A.8). As for the maser emission, we have identified both a $\mathrm{H}_{2} \mathrm{O}$ and a $\mathrm{CH}_{3} \mathrm{OH}$ maser feature associated with the brightest source \#83. This source has been studied in previous works (e.g., Roueff et al. 2006; Cutri \& et al. 2012; Azatyan et al. 2016; Stecklum et al. 2017), in some of them named RAFGL 7009S. The source is detected in the near-infrared together with two other objects separated by about $10^{\prime \prime}$, and surrounded by a diffuse and extended structure (see e.g., Stecklum et al. 2017).

López-Sepulcre et al. (2010) and Sánchez-Monge et al. (2013d) report on molecular outflow emission in the region. The blue and red contours in Fig. A.8 show the $\mathrm{SiO}(2-1)$ blue-shifted and red-shifted outflow emission. Both radio continuum sources (\#83 and \#95) are located close to the center of the outflow. Although this region was not included in the Cyganowski et al. (2008, 2009, 2011) surveys, we have identified a bright $4.5 \mu \mathrm{m}$ source in the Spitzer/GLIMPSE data. However, the source is located at the edge of the area surveyed by Spitzer, which prevents a detailed characterization of its infrared emission. The association of the source $\# 83$ with a $6.7 \mathrm{GHz} \mathrm{CH}_{3} \mathrm{OH}$ maser emission suggests that this source harbors a massive YSO together with its central location within the outflow and its association with a $\mathrm{H}_{2} \mathrm{O}$ maser feature hints towards this source is the radio jet that drives the outflow. The almost flat spectral index may indicate that this is a fully ionized radio jet. However, further observations in different frequency bands may help to better narrow down the spectral index and the status of radio continuum emission.

\section{G24.08+0.0M2 (\#96)}

We have identified fourteen radio continuum sources in the region G24.08+0.0 M2, one of which, source \#96, is detected in both frequency bands and has a negative spectral index $(-0.84 \pm$ +0.08 , see Fig. A.9). The outflow activity in the region has been studied by López-Sepulcre et al. (2011) and Sánchez-Monge et al. (2013d) who found molecular outflow in different tracers. However, this outflow is not spatially related to any of the radio continuum sources identified in this work. Also, this source could be a background source.

\section{G24.33+0.1M1 (\#110)}

In the region $\mathrm{G} 24.33+0.1 \mathrm{M} 1$ we identified a radio continuum source (\#110) located in the center of our field of view and detected in both frequency bands (see Fig. A.10). This source has a positive spectral index of $+0.73 \pm 0.35$, which is consistent with thermal emission. We have also detected maser features of both $\mathrm{H}_{2} \mathrm{O}$ and $\mathrm{CH}_{3} \mathrm{OH}$ associated with the continuum source. In addition, other authors have reported the presence of $\mathrm{OH}$ masers towards this object (see e.g., Caswell \& Green 2011). Rathborne et al. (2007) studied this source in the millimeter regime, and identified a singular compact source with a rich chemistry characteristic of hot molecular cores. Sánchez-Monge et al. (2013d) reported molecular outflow activity, with the source \#110 at its
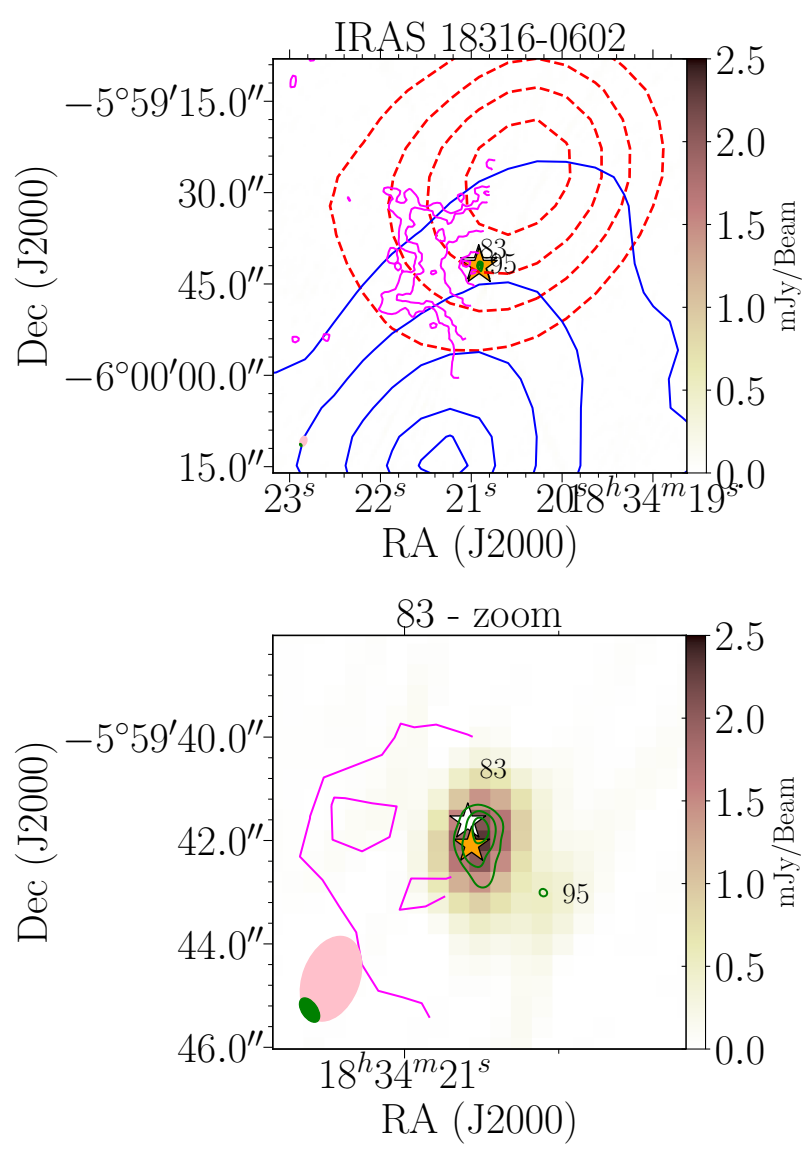

Fig. A.8. VLA C band $(6 \mathrm{~cm})$ continuum emission map of the radio jet candidates \#83 and \#95 located in the region IRAS 18316-1602. A close-up view of the two radio sources is shown in the bottom panel. The green contour levels of the $\mathrm{K}$ band $(1.3 \mathrm{~cm})$ continuum emission are 3, 5, 9 and 11 times $27 \mu \mathrm{Jy}_{\text {beam }}{ }^{-1}$. The magenta contours show the Spitzer/GLIMPSE $4.5 \mu \mathrm{m}$ emission (note that half of the region was not covered in the mapped area). The blue- and red-shifted outflow lobed of $\mathrm{SiO}(2-1)$ are shown as blue-solid and red-dashed contours, respectively (see Sánchez-Monge et al. 2013d). The pink and green ellipses are the beam sizes of the $\mathrm{C}$ and $\mathrm{K}$ bands, respectively. The white and orange stars mark the location of the $\mathrm{H}_{2} \mathrm{O}$ and $\mathrm{CH}_{3} \mathrm{OH}$ masers, respectively (see Table 2).

geometric center. Overall, this source is one of the best candidates for a thermal radio jet.

\section{G24.60+0.1M1 (\#119)}

As for \#119, the only information we have is that it is associated with an EGO. The nearest studied object is a extended $\mathrm{H}_{2}$ emission that is $19^{\prime \prime}$ away from \#119 (Froebrich et al. 2015). We can only say that we cannot determine the spectral index because the source is outside the primary beam of the $\mathrm{K}$ band images, but we propose that \#119 is a radio jet.

\section{G24.60+0.1M2 (\#136)}

Source \#136 is detected only in the K band, resulting in a spectral index limit $(>+1.67)$ that is consistent with thermal emission. The source is associated with $\mathrm{H}_{2} \mathrm{O}$ maser emission. This source, although not directly associated with one EGO, is located in the vicinity of G24.63+0.15 reported by Cyganowski et al. (2008, see green circle in Fig. A.11). Rathborne et al. (2007) suggest 


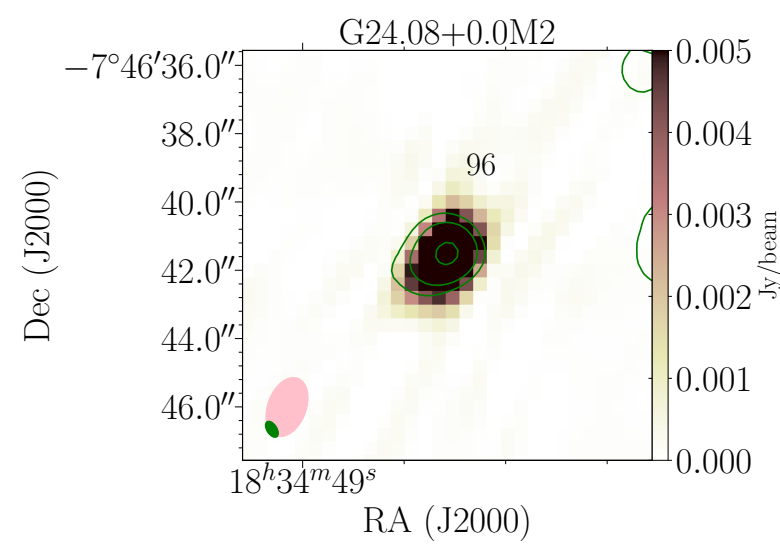

Fig. A.9. VLA C band $(6 \mathrm{~cm})$ continuum emission map of the radio source \#96 located in the region G24.08+0.0M2. The green contour levels of the $\mathrm{K}$ band $(1.3 \mathrm{~cm})$ continuum emission are 3,5 and 9 times $20 \mu \mathrm{Jy}_{\text {beam }^{-1}}$. The pink and green ellipses are the beam sizes of the $\mathrm{C}$ and $\mathrm{K}$ bands, respectively.

that the main dense condensation, hosting source \#136, may contain several condensations, referred to as G024.60+00.08 MM1 (A, B, and C). Our radio continuum source appears to be related to component $\mathrm{C}$ which is an IRDC condensation. Further observations of this object are necessary to confirm its possible nature as a radio jet.

\section{G34.43+0.2M3 (\#137 and \#139)}

Our radio continuum observations towards the region $\mathrm{G} 34.43+0.2 \mathrm{M} 3$ have led to the discovery of six radio continuum sources, although most of them are located far from the central region studied in Sánchez-Monge et al. (2013d). The brightest source is \#137, which is about $13^{\prime}$ from the phase center of our observations, i.e., the source is outside the primary beam responses of the VLA antennas on both bands. This object is so bright that it is detected in both the $\mathrm{C}$ and $\mathrm{K}$ bands. At $6 \mathrm{~cm}$, the source appears as a comet-like structure (see Fig. A.12), which resembles cometary $\mathrm{H}_{\text {II }}$ regions. The $1.3 \mathrm{~cm}$ continuum emission also shows an arc-shaped structure shifted to the east, probably tracing the head of the cometary object. This source, referred to in the literature as G34.26+0.15, has been studied by other authors who report the presence of two hyper-compact $\mathrm{H}_{\text {II }}$ regions (A and $\mathrm{B}$ ) and one ultra-compact $\mathrm{H}$ II region (C), all marked in the Figure (see also Reid \& Ho 1985; Gaume et al. 1994; Sewiło et al. 2011). Various studies (e.g., Hatchell et al. 2001; Liu et al. 2013) have reported the presence of outflow activity in this region, but no information on the outflow energetics such as the outflow momentum rate is reported. Despite this source is associated with an EGO (Cyganowski et al. 2008) and with a molecular outflow, the bright emission together with previous studies suggests that a major fraction of the radio continuum emission we have detected is originated in a $\mathrm{H}_{\mathrm{II}}$ region rather than in a radio jet. Source \#139 in the region, is also classified as a radio jet candidate in Table 3. However, the only information we have this object is its association with an EGO (i.e., G34.41+0.24, Cyganowski et al. 2008). Shepherd et al. (2004) suggest that the embedded object (G34.4MM) appears to be a massive B2 protostar at an early stage of evolution. This region is also associated with the $\mathrm{H}_{2} \mathrm{O}$ maser activity (Cyganowski et al. 2013), which may favor a radio
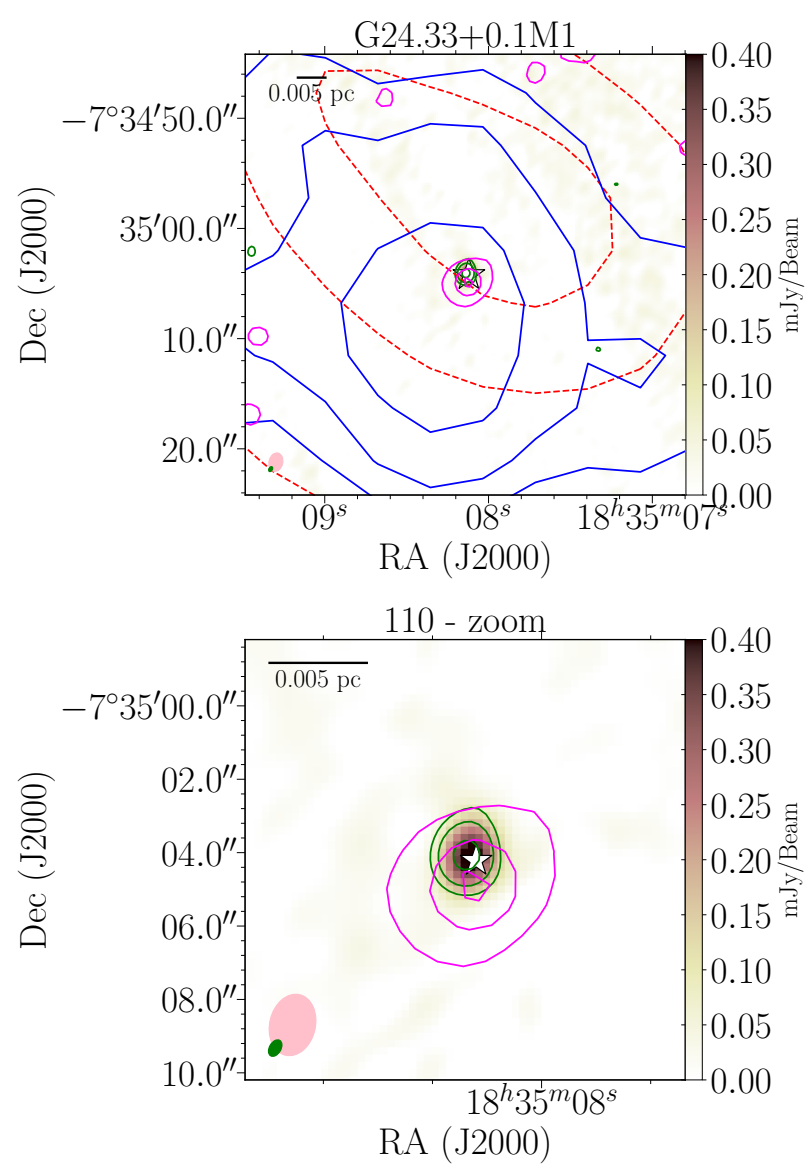

Fig. A.10. VLA C band $(6 \mathrm{~cm})$ continuum emission map of the radio jet candidate \#110 located in the region G24.33+0.1 M1. A close-up view of the radio source is shown in the bottom panel. The green contour levels of the $\mathrm{K}$ band $(1.3 \mathrm{~cm})$ continuum emission are 3,5 and 9 times $7 \mu \mathrm{Jy}_{\text {beam }}{ }^{-1}$. The blue- and red-shifted outflow lobed of $\mathrm{SiO}$ $(2-1)$ are shown as blue-solid and red-dashed contours, respectively (see Sánchez-Monge et al. 2013d). The pink and green ellipses are the beam sizes of the $\mathrm{C}$ and $\mathrm{K}$ bands, respectively. The white star marks the location of the $\mathrm{H}_{2} \mathrm{O}$ (see Table 2).

jet origin for the detected radio continuum emission. Further observations are needed to better constrain its properties.

\section{IRAS 19095+0930 (\#143 and \#144)}

We have identified four radio continuum sources in the region IRAS 19095+0930, also known in the literature as G43.80-0.13). Two of these radio sources, \#143 and \#144, are located close to each other and in the center of a molecular outflow (Sánchez-Monge et al. 2013d, see also Figure A.13). The source \#143 has a brighter flux, is also clearly visible in the $\mathrm{K}$ band image, and is associated with $\mathrm{H}_{2} \mathrm{O}$ maser emission features.

This region has been studied in the past by different authors at different wavelengths (Kurtz et al. 1994; Lekht 2000; De Buizer et al. 2005). De Buizer et al. (2005) report a kidney-bean shape structure at mid-infrared wavelengths that match the radio continuum sources reported by Kurtz et al. (1994) refers to as a $\mathrm{H}$ II region. The object is also associated with $\mathrm{OH}$ masers. We have not found this source in the EGO catalogues (Cyganowski et al. 2008, 2009), but we have identified a $4.5 \mu \mathrm{m}$ source associated with \#143. No $4.5 \mu \mathrm{m}$ infrared source appears to be associated with the eastern source \#144. 


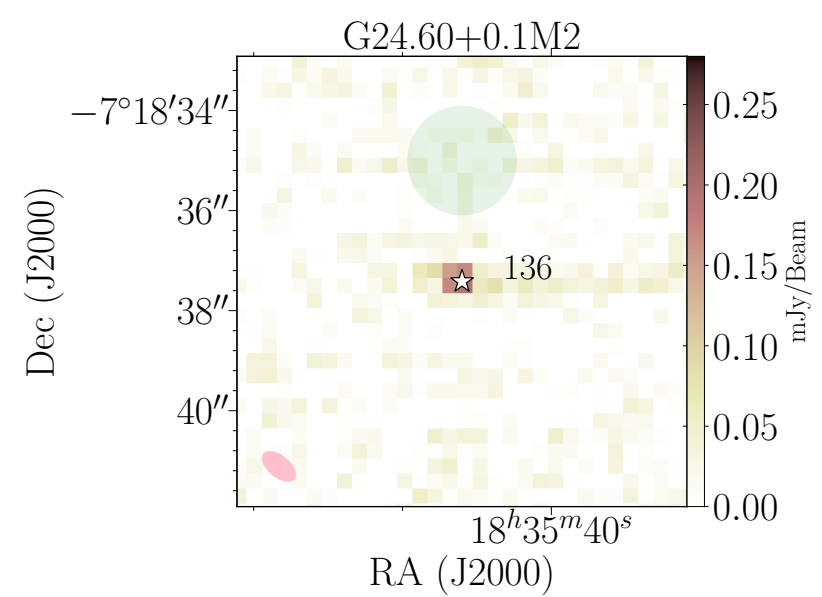

Fig. A.11. VLA K band $(1.3 \mathrm{~cm})$ continuum emission map of the radio jet candidate \#136 located in the region G24.60+0.1 M2. The green circle with a radius of $\sim 4^{\prime \prime}$ marks EGO G24.63+0.15, reported by Cyganowski et al. (2008). The pink ellipse is the beam size of the $\mathrm{K}$ band. The white star marks the location of the $\mathrm{H}_{2} \mathrm{O}$ (see Table 2).

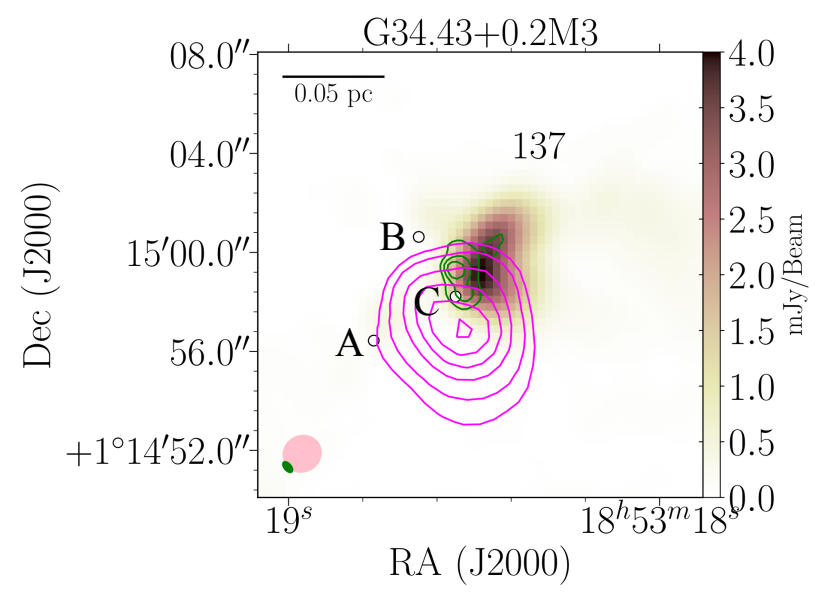

Fig. A.12. VLA C band $(6 \mathrm{~cm})$ continuum emission map of the radio jet candidate \#137 located in the region G34.43+0.2 M3. The green contour levels of the $\mathrm{K}$ band $(1.3 \mathrm{~cm})$ continuum emission are 3,5 and 7 times $20 \mu \mathrm{Jy}$ beam $^{-1}$. The magenta contours show the Spitzer/GLIMPSE $4.5 \mu \mathrm{m}$ emission. The pink and green ellipses are the beam sizes of the $\mathrm{C}$ and $\mathrm{K}$ bands, respectively. The white circles (A, $\mathrm{B}$, and C) mark the position of $2 \mathrm{~mm}$ continuum sources reported by Gasiprong et al. (2002).

We derive a spectral index of $+1.12 \pm 0.04$ for \#143, which is consistent with thermal emission. This, together with its location at the geometrical center of the outflow, and its association with masers may suggest that \#143 is a good radio jet candidate. However, the large radio continuum flux of this source seem to not be consistent with the typical properties of other radio jets (see Figs. 3 and 5). This might mean that this source is in a transition phase from a radio jet to an $\mathrm{H}$ II region. However, this requires further investigation.

\section{Appendix B: Catalog of the continuum sources}

In the following tables and figures, we provide information on the properties of the radio continuum sources detected in the VLA observations presented in this work. In Table B.1, we list the coordinates of the 146 radio continuum sources together with their flux density, intensity peak and deconvolved size at $6 \mathrm{~cm}$
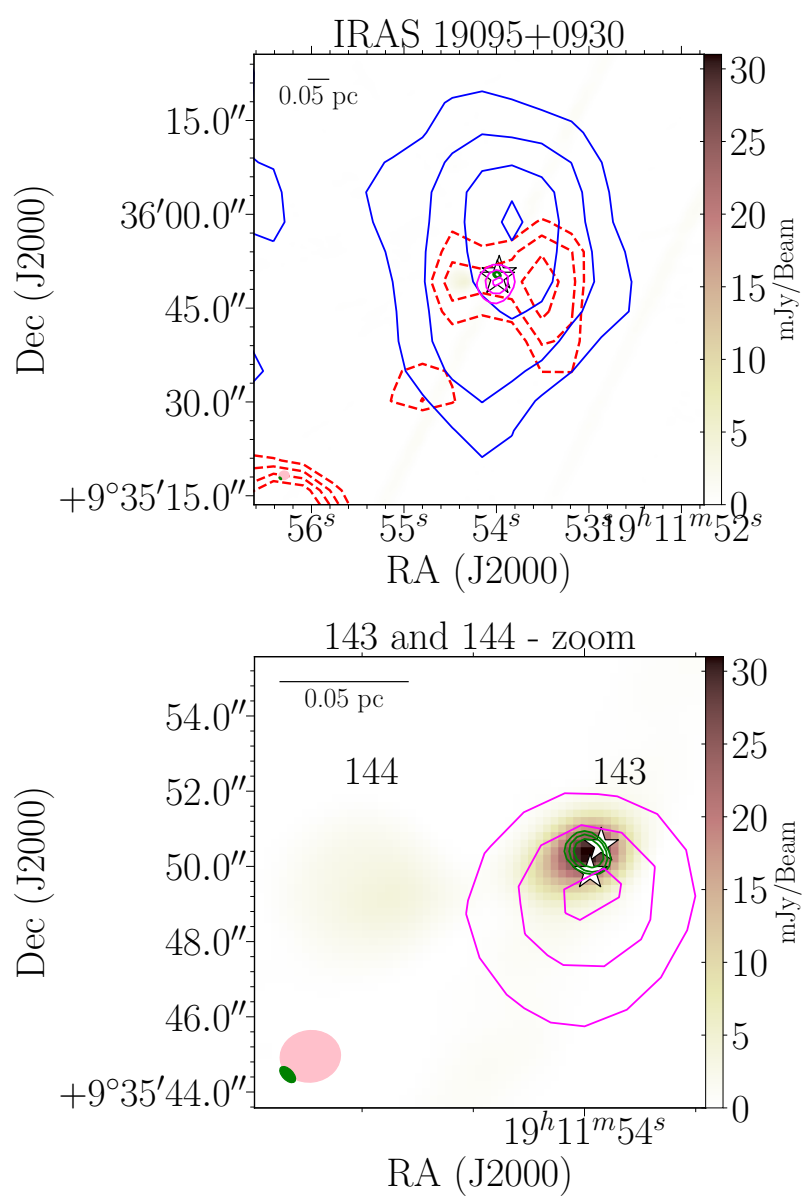

Fig. A.13. VLA C band $(6 \mathrm{~cm})$ continuum emission map of the radio sources \#143 and \#144 located in the region IRAS 19095+0930. The green contour levels of the $\mathrm{K}$ band $(1.3 \mathrm{~cm})$ continuum emission are 3 , 5 and 9 times $2 \mathrm{mJy}^{-1}$ beam $^{-1}$. The blue- and red-shifted outflow lobed of $\mathrm{SiO}(2-1)$ are shown as blue-solid and red-dashed contours, respectively (see Sánchez-Monge et al. 2013d). The magenta contours show the Spitzer/GLIMPSE $4.5 \mu \mathrm{m}$ emission. The pink and green ellipses are the beam sizes of the $\mathrm{C}$ and $\mathrm{K}$ bands, respectively. The white and orange stars mark the of the $\mathrm{H}_{2} \mathrm{O}$ and $\mathrm{CH}_{3} \mathrm{OH}$ masers (see Table 2).

( $\mathrm{C}$ band) and $1.3 \mathrm{~cm}$ ( $\mathrm{K}$ band). The fluxes and intensities are corrected by the primary beam response of the VLA antennas. For sources outside the $\mathrm{C}$ band primary beam (listed as "oC" in column (11) of Table B.1) the primary beam correction is not reliable and the flux has to be taken with caution. Similarly, for sources located outside the $\mathrm{K}$ band primary beam (labelled as "oK" in the Table), the $\mathrm{K}$ band flux has to be taken with caution. The last column of the Table lists the spectral index, $\alpha$. For sources with no reliable flux estimate at one of the bands, we do not determine the spectral index. For sources detected at both frequency bands ( $\mathrm{C}$ and $\mathrm{K}$ bands), the spectral index has been determined using the fluxes determined after creating images with a common $u v$-range (see Sect. 3.2 for more details). In Table B.2, we list the observed and deconvolved source sizes of all the detected sources. The source sizes are determined as $\sqrt{\theta_{\text {major }} \times \theta_{\text {minor }}}$, where $\theta_{\text {major }}$ and $\theta_{\text {minor }}$ are listed in Table B.2. We transform the angular size of each source into astronomical unit (au) using the distances listed in Table 1. We give the source size in Table B.1. Finally, in Table B.3, we list the intensities, flux densities and sizes determined from the images generated using a common $u v$-range at both $\mathrm{C}$ and $\mathrm{K}$ bands. In Figs. B. 1 to 
Kavak et al.: A search for radio jets from massive young stellar objects

B.16, we present close-up views of the $\mathrm{C}$ and $\mathrm{K}$ band emission for the 146 detected continuum sources. 


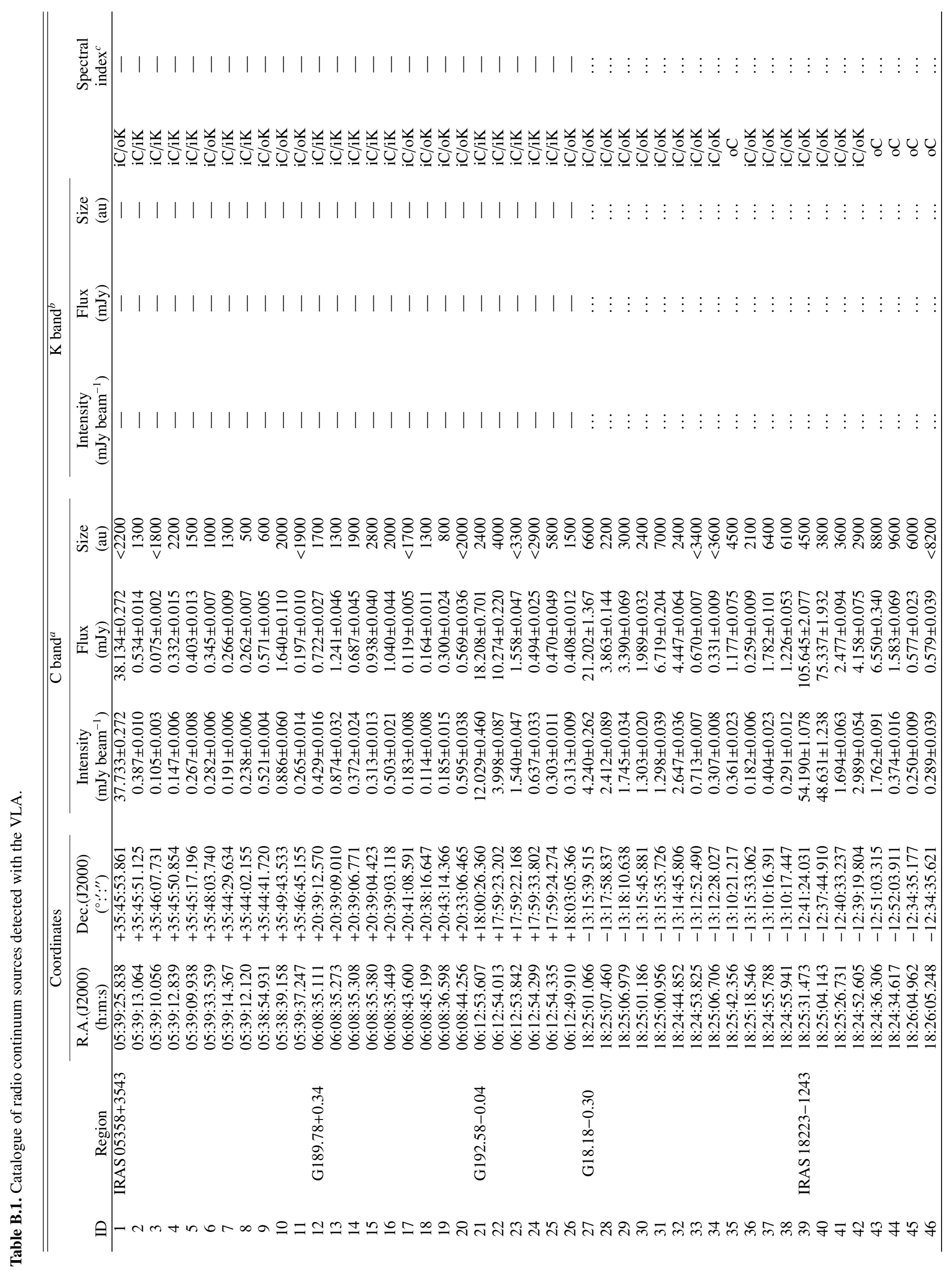




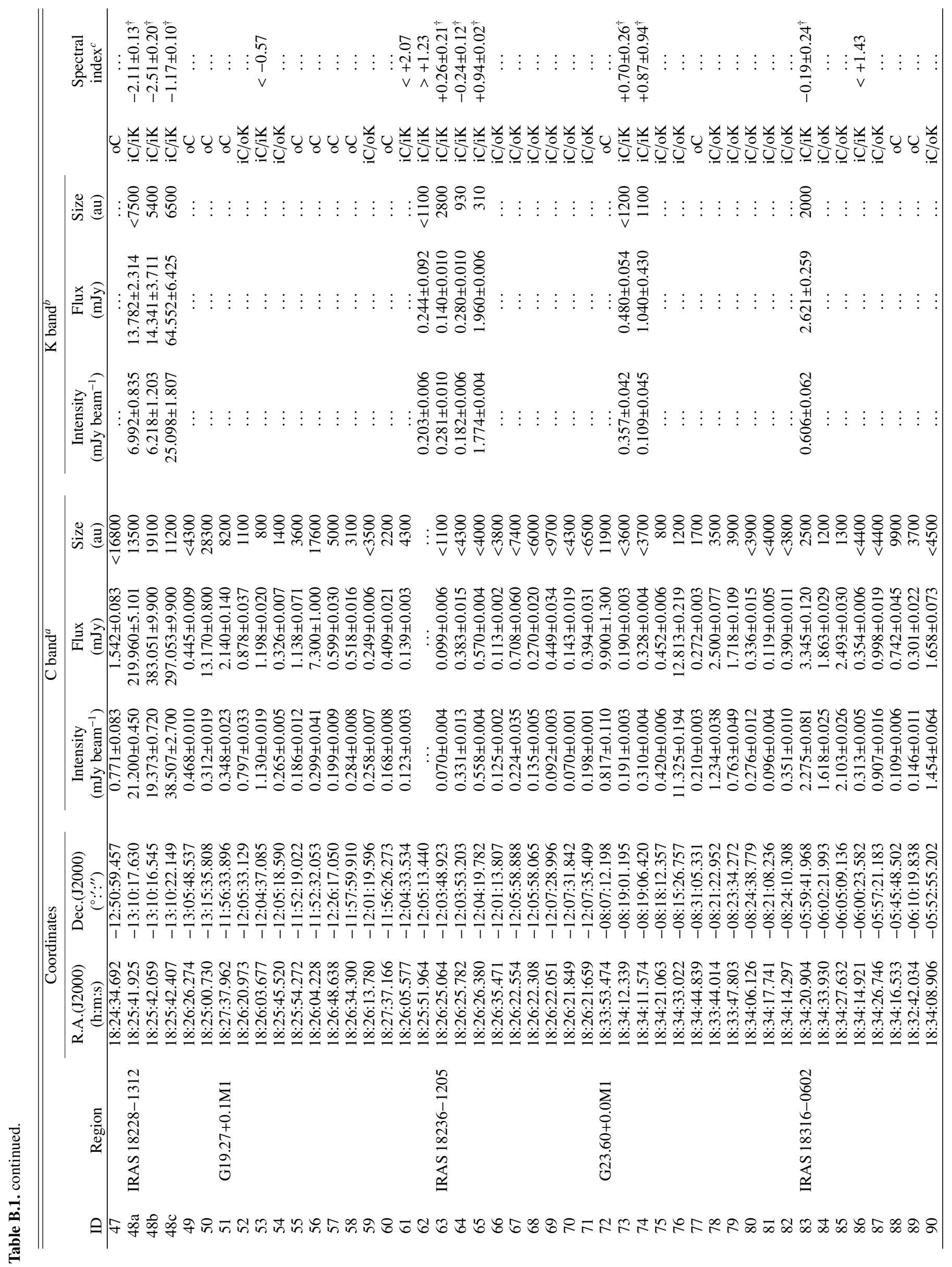




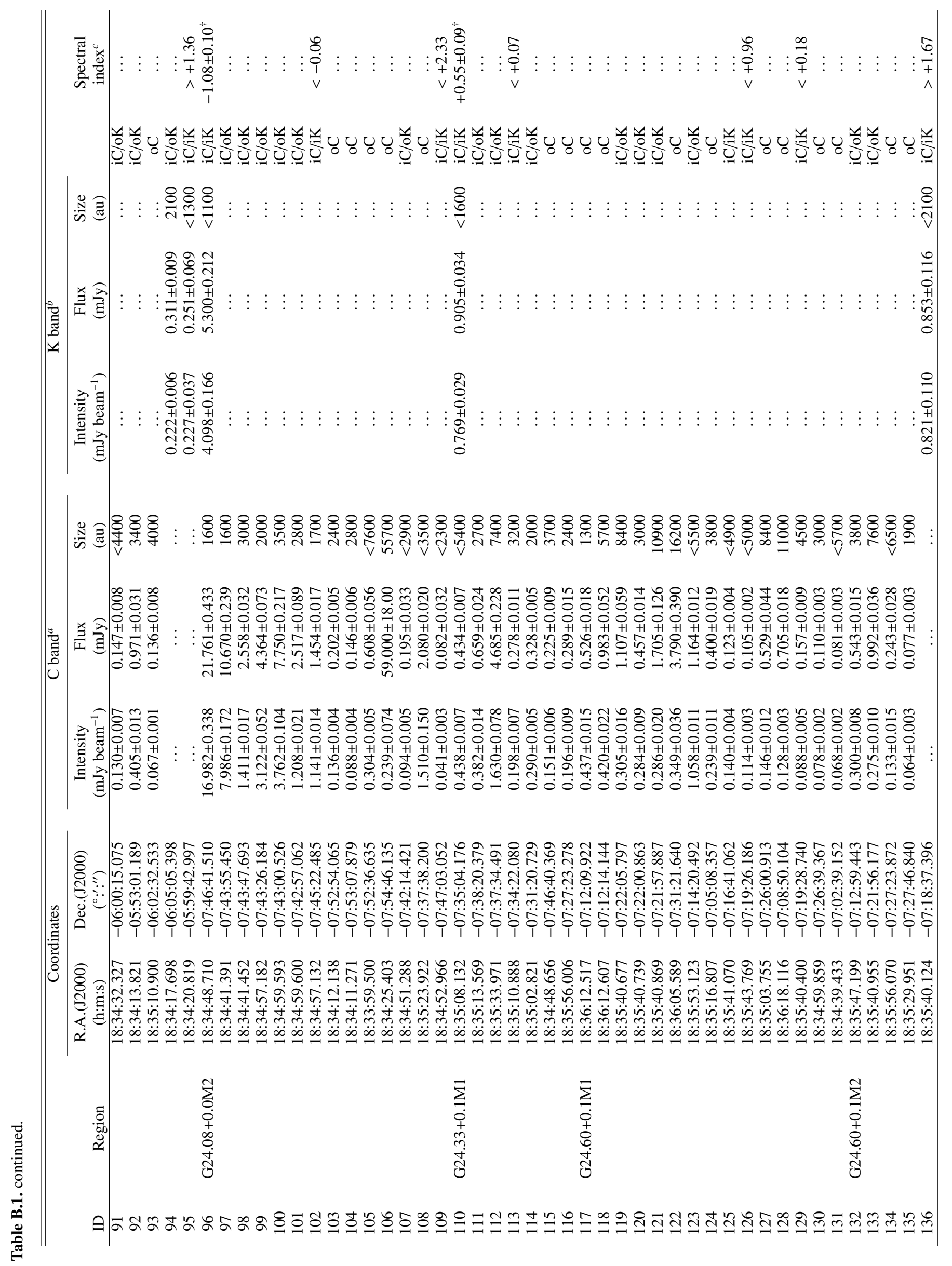




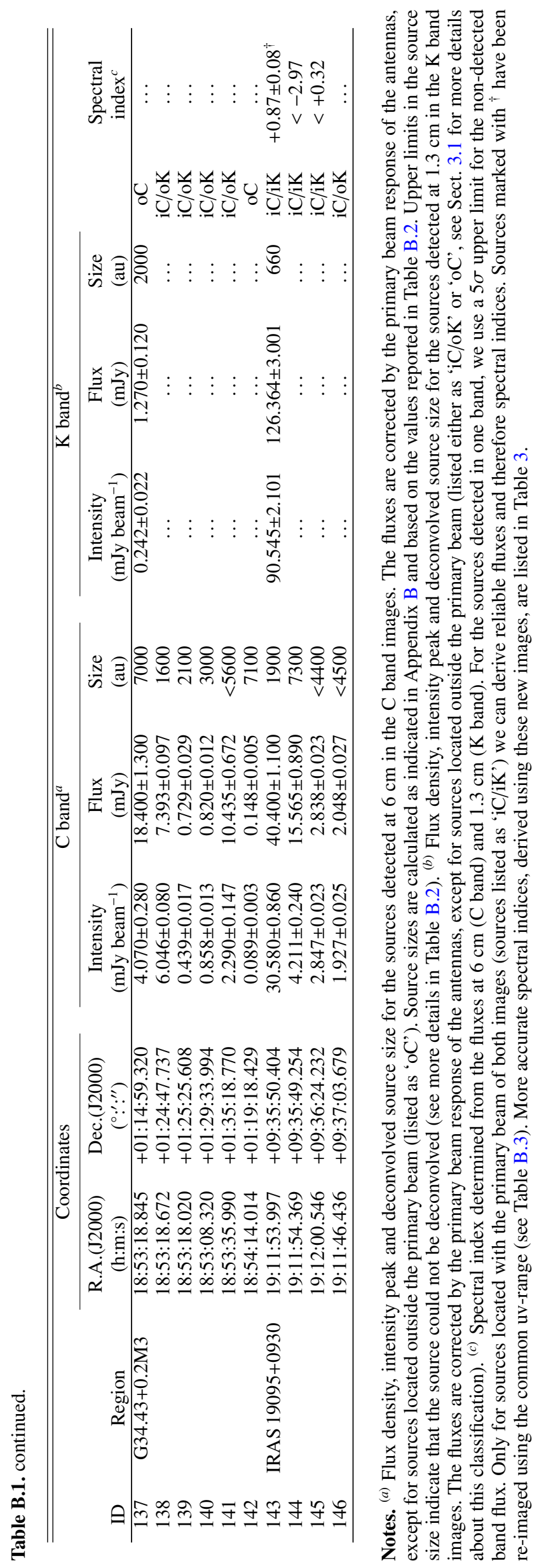


Table B.2. Observed and deconvolved angular sizes for the radio continuum sources detected in this work (see Table B.1)

\begin{tabular}{|c|c|c|c|c|c|c|c|}
\hline \multirow[b]{2}{*}{ ID } & \multirow[b]{2}{*}{ Region } & \multicolumn{3}{|c|}{ Observed source size } & \multicolumn{3}{|c|}{ Deconvolved diameter } \\
\hline & & $\begin{array}{l}\text { Major axis } \\
\text { (") }\end{array}$ & $\begin{array}{l}\text { Minor axis } \\
\left({ }^{\prime \prime}\right)\end{array}$ & $\begin{array}{l}\text { PA } \\
\left({ }^{\circ}\right)\end{array}$ & $\begin{array}{l}\text { Major axis } \\
\left({ }^{\prime \prime}\right)\end{array}$ & $\begin{array}{l}\text { Minor axis } \\
\left({ }^{\prime \prime}\right)\end{array}$ & $\begin{array}{l}\text { PA } \\
\left({ }^{\circ}\right)\end{array}$ \\
\hline \multicolumn{8}{|c|}{ Observed and deconvolved source sizes for $\mathrm{C}$ band detections } \\
\hline 1 & IRAS $05358+3543$ & $1.269 \pm 0.009$ & $1.239 \pm 0.009$ & $53.0 \pm 3.8$ & & & \\
\hline 2 & & $1.532 \pm 0.034$ & $1.402 \pm 0.034$ & $106.7 \pm 3.4$ & $0.894 \pm 0.063$ & $0.633 \pm 0.085$ & $114.9 \pm 9.9$ \\
\hline 3 & & $1.113 \pm 0.044$ & $1.001 \pm 0.043$ & $155.4 \pm 5.2$ & & & \\
\hline 4 & & $2.411 \pm 0.058$ & $1.455 \pm 0.057$ & $120.4 \pm 1.1$ & $2.069 \pm 0.069$ & $0.730 \pm 0.124$ & $121.1 \pm 1.2$ \\
\hline 5 & & $1.667 \pm 0.041$ & $1.409 \pm 0.041$ & $104.5 \pm 2.0$ & $1.105 \pm 0.065$ & $0.654 \pm 0.098$ & $108.5 \pm 3.8$ \\
\hline 6 & & $1.412 \pm 0.028$ & $1.349 \pm 0.027$ & $0.1 \pm 7.5$ & $0.690 \pm 0.066$ & $0.472 \pm 0.094$ & $169.0 \pm 9.0$ \\
\hline 7 & & $1.520 \pm 0.046$ & $1.424 \pm 0.044$ & $145.4 \pm 6.4$ & $0.899 \pm 0.082$ & $0.644 \pm 0.106$ & $147.1 \pm 6.4$ \\
\hline 8 & & $1.330 \pm 0.035$ & $1.286 \pm 0.034$ & $168.0 \pm 9.0$ & $0.512 \pm 0.758$ & $0.215 \pm 1.011$ & $160.0 \pm 9.0$ \\
\hline 9 & & $1.357 \pm 0.012$ & $1.255 \pm 0.012$ & $76.4 \pm 1.4$ & $0.490 \pm 0.035$ & $0.249 \pm 0.076$ & $85.0 \pm 9.0$ \\
\hline 10 & & $1.918 \pm 0.083$ & $1.502 \pm 0.086$ & $65.3 \pm 3.1$ & $1.440 \pm 0.110$ & $0.870 \pm 0.160$ & $65.6 \pm 3.8$ \\
\hline 11 & & $1.089 \pm 0.070$ & $1.065 \pm 0.068$ & $135.0 \pm 9.0$ & & & \\
\hline 12 & G189.78+0.34 & $1.673 \pm 0.041$ & $1.397 \pm 0.048$ & $12.0 \pm 2.2$ & $1.095 \pm 0.070$ & $0.860 \pm 0.089$ & $0.5 \pm 9.6$ \\
\hline 13 & & $1.578 \pm 0.041$ & $1.249 \pm 0.048$ & $14.6 \pm 1.8$ & $0.934 \pm 0.074$ & $0.602 \pm 0.115$ & $6.7 \pm 9.5$ \\
\hline 14 & & $1.781 \pm 0.072$ & $1.441 \pm 0.083$ & $10.1 \pm 3.9$ & $1.250 \pm 0.110$ & $0.930 \pm 0.150$ & $1.7 \pm 9.4$ \\
\hline 15 & & $2.461 \pm 0.073$ & $1.688 \pm 0.050$ & $158.3 \pm 2.0$ & $2.161 \pm 0.088$ & $1.194 \pm 0.079$ & $154.2 \pm 2.7$ \\
\hline 16 & & $1.920 \pm 0.050$ & $1.493 \pm 0.050$ & $156.9 \pm 1.8$ & $1.525 \pm 0.068$ & $0.880 \pm 0.096$ & $148.3 \pm 3.9$ \\
\hline 17 & & $1.014 \pm 0.052$ & $0.892 \pm 0.061$ & $18.6 \pm 6.4$ & & & \\
\hline 18 & & $1.598 \pm 0.078$ & $1.254 \pm 0.092$ & $21.7 \pm 4.9$ & $0.958 \pm 0.136$ & $0.629 \pm 0.226$ & $20.0 \pm 9.0$ \\
\hline 19 & & $1.959 \pm 0.091$ & $1.148 \pm 0.100$ & $174.7 \pm 2.0$ & $1.524 \pm 0.091$ & $0.136 \pm 0.100$ & $169.9 \pm 2.0$ \\
\hline 20 & & $1.382 \pm 0.071$ & $0.961 \pm 0.083$ & $16.5 \pm 2.4$ & & & $\ldots$ \\
\hline 21 & G192.58-0.04 & $1.741 \pm 0.046$ & $1.451 \pm 0.054$ & $25.8 \pm 2.2$ & $1.033 \pm 0.082$ & $0.827 \pm 0.106$ & $32.0 \pm 9.0$ \\
\hline 22 & & $2.524 \pm 0.064$ & $1.700 \pm 0.029$ & $59.1 \pm 1.5$ & $2.152 \pm 0.078$ & $1.116 \pm 0.052$ & $63.7 \pm 2.1$ \\
\hline 23 & & $1.454 \pm 0.036$ & $1.162 \pm 0.043$ & $30.3 \pm 1.8$ & $\ldots$ & $\ldots$ & $\ldots$ \\
\hline 24 & & $1.206 \pm 0.066$ & $1.073 \pm 0.072$ & $170.4 \pm 6.4$ & & & \\
\hline 25 & & $2.903 \pm 0.050$ & $2.375 \pm 0.038$ & $148.1 \pm 2.4$ & $2.614 \pm 0.060$ & $1.961 \pm 0.052$ & $143.0 \pm 3.1$ \\
\hline 26 & & $1.745 \pm 0.050$ & $1.247 \pm 0.042$ & $28.1 \pm 1.9$ & $1.043 \pm 0.064$ & $0.360 \pm 0.221$ & $32.0 \pm 4.4$ \\
\hline 27 & G18.18-0.30 & $3.480 \pm 0.102$ & $2.620 \pm 0.077$ & $47.0 \pm 1.4$ & $3.270 \pm 0.110$ & $2.030 \pm 0.110$ & $53.9 \pm 2.4$ \\
\hline 28 & & $1.884 \pm 0.040$ & $1.550 \pm 0.064$ & $174.8 \pm 2.4$ & $1.180 \pm 0.098$ & $0.657 \pm 0.172$ & $61.0 \pm 9.0$ \\
\hline 29 & & $2.210 \pm 0.025$ & $1.604 \pm 0.033$ & $11.5 \pm 0.8$ & $1.629 \pm 0.042$ & $0.820 \pm 0.085$ & $37.9 \pm 3.1$ \\
\hline 30 & & $1.962 \pm 0.017$ & $1.419 \pm 0.028$ & $165.8 \pm 0.8$ & $0.971 \pm 0.050$ & $0.890 \pm 0.053$ & $50.0 \pm 9.0$ \\
\hline 31 & & $3.244 \pm 0.057$ & $2.909 \pm 0.052$ & $151.8 \pm 4.5$ & $2.803 \pm 0.090$ & $2.645 \pm 0.091$ & $117.0 \pm 9.0$ \\
\hline 32 & & $1.926 \pm 0.016$ & $1.591 \pm 0.024$ & $179.5 \pm 0.7$ & $1.267 \pm 0.034$ & $0.711 \pm 0.058$ & $57.4 \pm 3.7$ \\
\hline 33 & & $1.637 \pm 0.011$ & $1.047 \pm 0.019$ & $166.6 \pm 0.3$ & $\ldots$ & $\ldots$ & $\ldots$ \\
\hline 34 & & $1.714 \pm 0.030$ & $1.148 \pm 0.049$ & $161.5 \pm 1.0$ & & & \\
\hline 35 & & $2.876 \pm 0.108$ & $2.066 \pm 0.072$ & $56.7 \pm 1.5$ & $2.660 \pm 0.120$ & $1.160 \pm 0.140$ & $62.3 \pm 2.0$ \\
\hline 36 & & $2.114 \pm 0.037$ & $1.225 \pm 0.061$ & $157.9 \pm 1.0$ & $1.224 \pm 0.073$ & $0.584 \pm 0.164$ & $147.5 \pm 6.1$ \\
\hline 37 & & $3.407 \pm 0.061$ & $2.362 \pm 0.099$ & $156.2 \pm 1.4$ & $2.938 \pm 0.074$ & $2.105 \pm 0.117$ & $152.7 \pm 3.1$ \\
\hline 38 & & $3.376 \pm 0.103$ & $2.275 \pm 0.068$ & $132.8 \pm 1.9$ & $3.000 \pm 0.130$ & $1.850 \pm 0.110$ & $123.8 \pm 3.9$ \\
\hline 39 & IRAS $18223-1243$ & $2.267 \pm 0.027$ & $1.865 \pm 0.034$ & $18.1 \pm 0.8$ & $1.715 \pm 0.043$ & $0.882 \pm 0.088$ & $54.0 \pm 3.4$ \\
\hline 40 & & $2.167 \pm 0.030$ & $1.550 \pm 0.048$ & $172.3 \pm 0.7$ & $1.163 \pm 0.073$ & $0.920 \pm 0.096$ & $33.0 \pm 9.0$ \\
\hline 41 & & $2.230 \pm 0.044$ & $1.423 \pm 0.072$ & $166.4 \pm 1.0$ & $1.178 \pm 0.085$ & $0.844 \pm 0.132$ & $168.0 \pm 9.0$ \\
\hline 42 & & $2.064 \pm 0.021$ & $1.461 \pm 0.034$ & $175.2 \pm 0.5$ & $1.053 \pm 0.055$ & $0.622 \pm 0.099$ & $41.7 \pm 7.7$ \\
\hline 43 & & $3.002 \pm 0.060$ & $2.684 \pm 0.098$ & $161.3 \pm 2.7$ & $2.440 \pm 0.120$ & $2.320 \pm 0.100$ & $91.0 \pm 9.0$ \\
\hline 44 & & $3.271 \pm 0.056$ & $2.803 \pm 0.078$ & $142.1 \pm 1.7$ & $2.810 \pm 0.081$ & $2.401 \pm 0.104$ & $116.0 \pm 9.0$ \\
\hline 45 & & $2.538 \pm 0.046$ & $1.970 \pm 0.075$ & $158.5 \pm 1.5$ & $1.748 \pm 0.088$ & $1.538 \pm 0.113$ & $129.0 \pm 9.0$ \\
\hline 46 & & $2.548 \pm 1.973$ & $1.973 \pm 0.091$ & $157.0 \pm 7.0$ & $\ldots$ & $\ldots$ & $\ldots$ \\
\hline 47 & & $5.050 \pm 0.250$ & $4.120 \pm 0.200$ & $143.0 \pm 9.0$ & & & $\ldots$ \\
\hline $48 \mathrm{a}$ & IRAS 18228-1312 & $6.450 \pm 0.144$ & $3.501 \pm 0.070$ & $4.3 \pm 1.2$ & $6.189 \pm 0.153$ & $3.268 \pm 0.080$ & $5.7 \pm 1.5$ \\
\hline $48 b$ & & $7.250 \pm 0.270$ & $5.940 \pm 0.220$ & $46.1 \pm 7.4$ & $7.120 \pm 0.290$ & $5.670 \pm 0.250$ & $49.0 \pm 8.0$ \\
\hline $48 c$ & & $4.610 \pm 0.340$ & $3.640 \pm 0.250$ & $65.0 \pm 9.0$ & $4.450 \pm 0.370$ & $3.130 \pm 0.320$ & $67.0 \pm 9.0$ \\
\hline 49 & & $1.784 \pm 0.025$ & $1.160 \pm 0.040$ & $163.8 \pm 0.7$ & & & $\ldots$ \\
\hline 50 & & $11.100 \pm 0.080$ & $8.271 \pm 0.107$ & $140.4 \pm 0.5$ & $10.960 \pm 0.082$ & $8.164 \pm 0.109$ & $139.5 \pm 0.6$ \\
\hline 51 & G19.27+0.1M1 & $4.790 \pm 0.110$ & $2.980 \pm 0.130$ & $167.3 \pm 1.7$ & $4.380 \pm 0.120$ & $2.730 \pm 0.150$ & $167.9 \pm 2.2$ \\
\hline 52 & & $2.071 \pm 0.050$ & $1.231 \pm 0.082$ & $163.8 \pm 1.7$ & $0.694 \pm 1.257$ & $0.317 \pm 0.872$ & $161.0 \pm 9.0$ \\
\hline 53 & & $2.018 \pm 0.021$ & $1.218 \pm 0.034$ & $164.9 \pm 0.7$ & $0.518 \pm 1.433$ & $0.258 \pm 0.931$ & $173.0 \pm 9.0$ \\
\hline 54 & & $2.060 \pm 0.028$ & $1.387 \pm 0.042$ & $171.9 \pm 1.3$ & $0.889 \pm 1.063$ & $0.391 \pm 0.797$ & $36.0 \pm 9.0$ \\
\hline 55 & & $4.480 \pm 0.140$ & $3.170 \pm 0.110$ & $95.3 \pm 2.9$ & $4.290 \pm 0.150$ & $0.550 \pm 0.150$ & $91.0 \pm 9.0$ \\
\hline 56 & & $8.700 \pm 0.260$ & $6.540 \pm 0.260$ & $100.9 \pm 4.2$ & $8.590 \pm 0.280$ & $6.280 \pm 0.280$ & $99.3 \pm 4.4$ \\
\hline 57 & & $3.057 \pm 0.094$ & $2.282 \pm 0.092$ & $141.4 \pm 3.5$ & $2.480 \pm 0.140$ & $1.780 \pm 0.170$ & $124.0 \pm 9.0$ \\
\hline 58 & & $2.447 \pm 0.037$ & $1.724 \pm 0.059$ & $175.3 \pm 1.3$ & $1.571 \pm 0.077$ & $1.125 \pm 0.116$ & $19.6 \pm 9.9$ \\
\hline 59 & & $1.951 \pm 0.033$ & $1.147 \pm 0.053$ & $159.8 \pm 1.2$ & $\ldots$ & $\ldots$ & $\ldots$ \\
\hline
\end{tabular}


Table B.2. continued.

\begin{tabular}{|c|c|c|c|c|c|c|c|}
\hline \multirow[b]{2}{*}{ ID } & \multirow[b]{2}{*}{ Region } & \multicolumn{3}{|c|}{ Observed source size } & \multicolumn{3}{|c|}{ Deconvolved diameter } \\
\hline & & $\begin{array}{l}\text { Major axis } \\
\left({ }^{\prime \prime}\right)\end{array}$ & $\begin{array}{c}\text { Minor axis } \\
\left({ }^{\prime \prime}\right)\end{array}$ & $\begin{array}{l}\text { PA } \\
\left({ }^{\circ}\right)\end{array}$ & $\begin{array}{l}\text { Major axis } \\
\left({ }^{\prime \prime}\right)\end{array}$ & $\begin{array}{c}\text { Minor axis } \\
\left({ }^{\prime \prime}\right)\end{array}$ & $\begin{array}{l}\text { PA } \\
\left({ }^{\circ}\right)\end{array}$ \\
\hline 60 & & $2.300 \pm 0.140$ & $1.430 \pm 0.120$ & $170.1 \pm 3.6$ & $1.250 \pm 0.310$ & $0.730 \pm 0.480$ & $4.2 \pm 9.8$ \\
\hline 61 & & $2.950 \pm 0.100$ & $1.900 \pm 0.100$ & $152.8 \pm 2.5$ & $2.250 \pm 0.150$ & $1.430 \pm 0.170$ & $143.8 \pm 8.4$ \\
\hline 63 & IRAS 18236-1205 & $0.485 \pm 0.019$ & $0.393 \pm 0.021$ & $134.6 \pm 7.2$ & $\ldots$ & $\ldots$ & $\ldots$ \\
\hline 64 & & $2.229 \pm 0.061$ & $1.148 \pm 0.079$ & $170.1 \pm 1.5$ & $\ldots$ & $\ldots$ & $\ldots$ \\
\hline 65 & & $2.033 \pm 0.012$ & $1.112 \pm 0.017$ & $163.6 \pm 0.3$ & $\ldots$ & $\ldots$ & $\ldots$ \\
\hline 66 & & $1.785 \pm 0.044$ & $1.121 \pm 0.042$ & $156.1 \pm 1.6$ & $\ldots$ & $\ldots$ & $\ldots$ \\
\hline 67 & & $4.068 \pm 1.886$ & $1.886 \pm 0.093$ & $116.0 \pm 2.0$ & $\ldots$ & $\ldots$ & $\ldots$ \\
\hline 68 & & $2.633 \pm 0.156$ & $1.893 \pm 0.092$ & $138.0 \pm 6.0$ & $\ldots$ & $\ldots$ & $\ldots$ \\
\hline 69 & & $6.040 \pm 0.820$ & $2.180 \pm 0.210$ & $3.0 \pm 3.0$ & $\ldots$ & $\ldots$ & $\ldots$ \\
\hline 70 & & $1.840 \pm 0.170$ & $1.400 \pm 0.100$ & $126.0 \pm 9.0$ & $\ldots$ & $\ldots$ & $\ldots$ \\
\hline 71 & & $3.193 \pm 0.223$ & $1.823 \pm 0.093$ & $159.0 \pm 4.0$ & 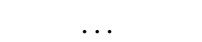 & & \\
\hline 72 & $\mathrm{G} 23.60+0.0 \mathrm{M} 1$ & $5.110 \pm 0.210$ & $4.960 \pm 0.210$ & $114.0 \pm 9.0$ & $4.930 \pm 0.290$ & $4.660 \pm 0.300$ & $86.0 \pm 9.0$ \\
\hline 73 & & $1.770 \pm 0.029$ & $1.175 \pm 0.035$ & $160.1 \pm 1.4$ & $\ldots$ & $\ldots$ & $\ldots$ \\
\hline 74 & & $1.793 \pm 0.021$ & $1.236 \pm 0.025$ & $160.3 \pm 1.0$ & $\ldots$ & $\ldots$ & $\ldots$ \\
\hline 75 & & $1.884 \pm 0.020$ & $1.198 \pm 0.026$ & $156.3 \pm 0.8$ & $0.444 \pm 1.410$ & $0.290 \pm 0.842$ & $105.0 \pm 9.0$ \\
\hline 76 & & $1.926 \pm 0.019$ & $1.230 \pm 0.032$ & $157.3 \pm 0.8$ & $0.541 \pm 0.083$ & $0.475 \pm 0.117$ & $141.0 \pm 9.0$ \\
\hline 77 & & $1.975 \pm 0.017$ & $1.372 \pm 0.027$ & $162.3 \pm 0.9$ & $0.847 \pm 0.061$ & $0.602 \pm 0.087$ & $35.0 \pm 9.0$ \\
\hline 78 & & $2.285 \pm 0.041$ & $1.856 \pm 0.057$ & $152.2 \pm 3.1$ & $1.497 \pm 0.095$ & $1.312 \pm 0.111$ & $88.0 \pm 9.0$ \\
\hline 79 & & $2.672 \pm 0.090$ & $1.760 \pm 0.116$ & $174.9 \pm 2.6$ & $2.010 \pm 0.140$ & $1.220 \pm 0.220$ & $8.9 \pm 9.8$ \\
\hline 80 & & $1.817 \pm 0.065$ & $1.405 \pm 0.084$ & $173.7 \pm 4.9$ & $\ldots$ & $\ldots$ & $\ldots$ \\
\hline 81 & & $1.828 \pm 0.051$ & $1.418 \pm 0.080$ & $169.0 \pm 4.0$ & $\ldots$ & $\ldots$ & $\ldots$ \\
\hline 82 & & $1.762 \pm 0.033$ & $1.324 \pm 0.054$ & $152.6 \pm 2.3$ & & & \\
\hline 83 & IRAS 18316-0602 & $1.987 \pm 0.041$ & $1.376 \pm 0.060$ & $174.1 \pm 1.9$ & $1.181 \pm 0.096$ & $0.592 \pm 0.228$ & $22.5 \pm 9.8$ \\
\hline 84 & & $1.741 \pm 0.018$ & $1.230 \pm 0.028$ & $163.2 \pm 1.1$ & $0.620 \pm 1.085$ & $0.251 \pm 0.838$ & $46.0 \pm 9.0$ \\
\hline 85 & & $1.759 \pm 0.014$ & $1.253 \pm 0.021$ & $165.1 \pm 0.9$ & $0.696 \pm 0.053$ & $0.283 \pm 0.184$ & $41.5 \pm 7.6$ \\
\hline 86 & & $1.683 \pm 0.021$ & $1.250 \pm 0.031$ & $163.2 \pm 1.7$ & $\ldots$ & $\ldots$ & $\ldots$ \\
\hline 87 & & $1.685 \pm 0.021$ & $1.215 \pm 0.032$ & $159.8 \pm 1.4$ & & & \\
\hline 88 & & $4.688 \pm 0.090$ & $2.687 \pm 0.094$ & $13.0 \pm 1.1$ & $4.434 \pm 0.099$ & $2.333 \pm 0.117$ & $16.2 \pm 1.5$ \\
\hline 89 & & $2.250 \pm 0.100$ & $1.690 \pm 0.110$ & $118.4 \pm 4.7$ & $1.790 \pm 0.160$ & $0.800 \pm 0.400$ & $98.0 \pm 9.0$ \\
\hline 90 & & $1.709 \pm 0.050$ & $1.240 \pm 0.074$ & $145.2 \pm 3.2$ & $\ldots$ & $\ldots$ & $\ldots$ \\
\hline 91 & & $1.521 \pm 0.081$ & $1.376 \pm 0.099$ & $172.0 \pm 9.0$ & . & 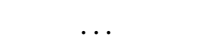 & $\ldots$ \\
\hline 92 & & $2.140 \pm 0.110$ & $1.500 \pm 0.110$ & $145.9 \pm 4.7$ & $1.380 \pm 0.230$ & $0.910 \pm 0.330$ & $124.0 \pm 9.0$ \\
\hline 93 & & $2.474 \pm 0.119$ & $1.509 \pm 0.075$ & $173.9 \pm 2.4$ & $1.840 \pm 0.180$ & $0.950 \pm 0.170$ & $4.0 \pm 7.9$ \\
\hline 96 & $\mathrm{G} 24.08+0.0 \mathrm{M} 2$ & $1.977 \pm 0.023$ & $1.317 \pm 0.036$ & $150.6 \pm 1.0$ & $0.942 \pm 0.068$ & $0.487 \pm 0.151$ & $118.6 \pm 8.7$ \\
\hline 97 & & $1.935 \pm 0.026$ & $1.402 \pm 0.039$ & $171.8 \pm 1.5$ & $1.008 \pm 0.078$ & $0.418 \pm 0.265$ & $37.1 \pm 7.8$ \\
\hline 98 & & $2.469 \pm 0.022$ & $1.492 \pm 0.022$ & $174.4 \pm 0.6$ & $1.759 \pm 0.035$ & $0.842 \pm 0.052$ & $6.8 \pm 1.8$ \\
\hline 99 & & $1.940 \pm 0.019$ & $1.464 \pm 0.030$ & $161.5 \pm 1.2$ & $0.942 \pm 0.054$ & $0.708 \pm 0.068$ & $59.0 \pm 9.0$ \\
\hline 100 & & $2.967 \pm 0.039$ & $1.410 \pm 0.050$ & $159.1 \pm 0.7$ & $2.357 \pm 0.050$ & $0.846 \pm 0.086$ & $159.1 \pm 1.0$ \\
\hline 101 & & $2.471 \pm 0.101$ & $1.714 \pm 0.054$ & $22.8 \pm 3.0$ & $2.020 \pm 0.140$ & $0.660 \pm 0.280$ & $39.1 \pm 5.2$ \\
\hline 102 & & $1.907 \pm 0.016$ & $1.358 \pm 0.022$ & $161.6 \pm 0.9$ & $0.776 \pm 0.049$ & $0.599 \pm 0.070$ & $50.0 \pm 9.0$ \\
\hline 103 & & $2.146 \pm 0.049$ & $1.399 \pm 0.053$ & $153.8 \pm 1.7$ & $1.190 \pm 0.100$ & $0.790 \pm 0.130$ & $140.0 \pm 9.0$ \\
\hline 104 & & $2.180 \pm 0.150$ & $1.540 \pm 0.110$ & $159.7 \pm 5.7$ & $1.230 \pm 0.270$ & $1.050 \pm 0.270$ & $162.0 \pm 9.0$ \\
\hline 105 & & $3.350 \pm 0.270$ & $2.830 \pm 0.210$ & $162.0 \pm 9.0$ & & & \\
\hline 106 & & $26.310 \pm 1.400$ & $19.000 \pm 0.530$ & $69.7 \pm 4.3$ & $26.280 \pm 1.400$ & $18.910 \pm 0.540$ & $69.7 \pm 4.3$ \\
\hline 107 & & $1.369 \pm 0.138$ & $1.020 \pm 0.072$ & $160.0 \pm 9.6$ & $\ldots$ & $\ldots$ & $\ldots$ \\
\hline 108 & & $1.540 \pm 1.289$ & $1.289 \pm 0.069$ & $10.0 \pm 9.1$ & $\ldots$ & $\ldots$ & $\ldots$ \\
\hline 109 & & $0.940 \pm 0.130$ & $0.940 \pm 0.130$ & $168.0 \pm 7.7$ & $\ldots$ & $\ldots$ & $\ldots$ \\
\hline 110 & $\mathrm{G} 24.33+0.1 \mathrm{M} 1$ & $1.661 \pm 0.021$ & $1.259 \pm 0.029$ & $166.9 \pm 0.7$ & $\ldots$ & $\ldots$ & $\ldots$ \\
\hline 111 & & $2.114 \pm 0.064$ & $1.722 \pm 0.047$ & $78.2 \pm 1.8$ & $1.708 \pm 0.081$ & $0.315 \pm 0.931$ & $78.0 \pm 9.0$ \\
\hline 112 & & $2.571 \pm 0.064$ & $2.361 \pm 0.081$ & $147.2 \pm 4.0$ & $2.070 \pm 0.110$ & $1.860 \pm 0.120$ & $103.0 \pm 9.0$ \\
\hline 113 & & $1.957 \pm 0.051$ & $1.511 \pm 0.066$ & $150.4 \pm 1.8$ & $1.120 \pm 0.110$ & $0.650 \pm 0.220$ & $121.0 \pm 9.0$ \\
\hline 114 & & $1.821 \pm 0.022$ & $1.314 \pm 0.030$ & $167.3 \pm 0.7$ & $0.670 \pm 0.064$ & $0.414 \pm 0.114$ & $170.0 \pm 9.0$ \\
\hline 115 & & $1.976 \pm 0.051$ & $1.584 \pm 0.068$ & $155.8 \pm 2.0$ & $1.120 \pm 0.120$ & $0.860 \pm 0.160$ & $121.0 \pm 9.0$ \\
\hline 116 & & $1.861 \pm 0.079$ & $1.673 \pm 0.072$ & $112.4 \pm 4.5$ & $1.321 \pm 0.079$ & $0.307 \pm 0.072$ & $87.9 \pm 4.5$ \\
\hline 117 & $\mathrm{G} 24.60+0.1 \mathrm{M} 1$ & $1.681 \pm 0.041$ & $1.456 \pm 0.056$ & $168.5 \pm 3.9$ & $0.800 \pm 0.873$ & $0.170 \pm 1.500$ & $82.0 \pm 9.0$ \\
\hline 118 & & $2.304 \pm 0.086$ & $2.068 \pm 0.067$ & $60.9 \pm 6.6$ & $1.940 \pm 0.110$ & $1.240 \pm 0.130$ & $71.6 \pm 6.6$ \\
\hline 119 & & $3.087 \pm 0.074$ & $2.396 \pm 0.081$ & $132.6 \pm 2.5$ & $2.708 \pm 0.096$ & $1.914 \pm 0.118$ & $122.5 \pm 5.1$ \\
\hline 120 & & $2.232 \pm 0.048$ & $1.467 \pm 0.052$ & $20.8 \pm 1.5$ & $1.636 \pm 0.076$ & $0.426 \pm 0.791$ & $35.0 \pm 9.0$ \\
\hline 121 & & $5.480 \pm 0.200$ & $2.210 \pm 0.110$ & $34.4 \pm 1.5$ & $5.280 \pm 0.210$ & $1.650 \pm 0.160$ & $35.9 \pm 1.7$ \\
\hline 122 & & $6.720 \pm 0.150$ & $3.290 \pm 0.150$ & $38.5 \pm 1.3$ & $6.570 \pm 0.160$ & $2.930 \pm 0.170$ & $39.6 \pm 1.4$ \\
\hline 123 & & $1.856 \pm 0.013$ & $1.205 \pm 0.018$ & $163.1 \pm 0.5$ & & & \\
\hline 124 & & $1.902 \pm 0.066$ & $1.788 \pm 0.073$ & $25.0 \pm 9.0$ & $1.380 \pm 0.130$ & $0.800 \pm 0.250$ & $71.0 \pm 9.0$ \\
\hline 125 & & $1.594 \pm 0.041$ & $1.122 \pm 0.056$ & $168.9 \pm 2.3$ & $\ldots$ & $\ldots$ & $\ldots$ \\
\hline 126 & & $1.708 \pm 0.042$ & $1.097 \pm 0.044$ & $162.8 \pm 1.8$ & $\ldots$ & & \\
\hline 127 & & $2.910 \pm 0.120$ & $2.530 \pm 0.130$ & $132.0 \pm 7.6$ & $2.530 \pm 0.170$ & $2.050 \pm 0.200$ & $114.0 \pm 9.0$ \\
\hline
\end{tabular}


Table B.2. continued.

\begin{tabular}{|c|c|c|c|c|c|c|c|}
\hline \multirow[b]{2}{*}{ ID } & \multirow[b]{2}{*}{ Region } & \multicolumn{3}{|c|}{ Observed source size } & \multicolumn{3}{|c|}{ Deconvolved diameter } \\
\hline & & $\begin{array}{l}\text { Major axis } \\
\left({ }^{\prime \prime}\right)\end{array}$ & $\begin{array}{c}\text { Minor axis } \\
\left({ }^{\prime \prime}\right)\end{array}$ & $\begin{array}{l}\text { PA } \\
\left({ }^{\circ}\right)\end{array}$ & $\begin{array}{c}\text { Major axis } \\
\left({ }^{\prime \prime}\right)\end{array}$ & $\begin{array}{c}\text { Minor axis } \\
\left({ }^{\prime \prime}\right)\end{array}$ & $\begin{array}{l}\text { PA } \\
\left({ }^{\circ}\right)\end{array}$ \\
\hline 128 & & $4.754 \pm 0.072$ & $2.358 \pm 0.042$ & $5.9 \pm 0.6$ & $4.462 \pm 0.078$ & $1.992 \pm 0.052$ & $7.2 \pm 0.8$ \\
\hline 129 & & $2.374 \pm 0.071$ & $1.524 \pm 0.096$ & $176.8 \pm 2.1$ & $1.690 \pm 0.110$ & $0.900 \pm 0.190$ & $1.1 \pm 5.6$ \\
\hline 130 & & $1.908 \pm 0.037$ & $1.501 \pm 0.047$ & $151.2 \pm 3.0$ & $1.115 \pm 0.097$ & $0.612 \pm 0.201$ & $118.0 \pm 9.0$ \\
\hline 131 & & $2.076 \pm 0.077$ & $1.161 \pm 0.061$ & $165.9 \pm 2.1$ & & & \\
\hline 132 & $\mathrm{G} 24.60+0.01 \mathrm{M} 2$ & $2.003 \pm 0.081$ & $1.537 \pm 0.046$ & $137.2 \pm 3.8$ & $1.400 \pm 0.130$ & $0.790 \pm 0.220$ & $103.0 \pm 9.0$ \\
\hline 133 & & $2.778 \pm 0.097$ & $2.206 \pm 0.101$ & $0.1 \pm 5.5$ & $2.310 \pm 0.150$ & $1.860 \pm 0.170$ & $17.0 \pm 9.0$ \\
\hline 134 & & $2.080 \pm 0.180$ & $1.490 \pm 0.140$ & $98.7 \pm 6.2$ & & & \\
\hline 135 & & $1.826 \pm 0.074$ & $1.119 \pm 0.078$ & $166.0 \pm 2.7$ & $0.813 \pm 0.843$ & $0.354 \pm 0.673$ & $4.7 \pm 9.8$ \\
\hline 137 & $\mathrm{G} 34.43+0.2 \mathrm{M} 3$ & $3.890 \pm 0.110$ & $2.660 \pm 0.110$ & $165.1 \pm 1.4$ & $3.580 \pm 0.120$ & $2.190 \pm 0.130$ & $166.2 \pm 1.7$ \\
\hline 138 & & $1.764 \pm 0.020$ & $1.587 \pm 0.020$ & $156.0 \pm 1.8$ & $0.898 \pm 0.045$ & $0.483 \pm 0.084$ & $172.0 \pm 5.7$ \\
\hline 139 & & $2.024 \pm 0.065$ & $1.596 \pm 0.054$ & $143.8 \pm 2.5$ & $1.287 \pm 0.087$ & $0.594 \pm 0.181$ & $149.0 \pm 5.0$ \\
\hline 140 & & $1.553 \pm 0.023$ & $1.880 \pm 0.061$ & $15.4 \pm 8.2$ & $1.390 \pm 0.100$ & $1.040 \pm 0.120$ & $21.0 \pm 8.5$ \\
\hline 141 & & $3.575 \pm 0.098$ & $1.410 \pm 0.024$ & $150.5 \pm 2.5$ & & & \\
\hline 142 & & $2.217 \pm 0.056$ & $2.918 \pm 0.098$ & $74.9 \pm 4.1$ & $3.240 \pm 0.110$ & $2.490 \pm 0.120$ & $72.7 \pm 4.9$ \\
\hline 143 & IRAS $19095+0930$ & $2.014 \pm 0.039$ & $1.445 \pm 0.044$ & $115.5 \pm 0.8$ & $1.250 \pm 0.350$ & $0.340 \pm 1.030$ & $124.0 \pm 9.0$ \\
\hline 144 & & $3.158 \pm 0.079$ & $2.577 \pm 0.091$ & $87.0 \pm 1.6$ & $2.724 \pm 0.090$ & $2.177 \pm 0.111$ & $84.3 \pm 3.7$ \\
\hline 145 & & $1.601 \pm 0.011$ & $1.370 \pm 0.013$ & $94.7 \pm 0.6$ & $\ldots$ & $\ldots$ & $\ldots$ \\
\hline 146 & & $1.715 \pm 0.018$ & $1.365 \pm 0.021$ & $105.1 \pm 0.6$ & $\ldots$ & $\ldots$ & $\ldots$ \\
\hline \multicolumn{8}{|c|}{ Observed and deconvolved source sizes for $\mathrm{K}$ band detections } \\
\hline $48 \mathrm{a}$ & IRAS $18228-1312$ & $2.950 \pm 0.390$ & $2.090 \pm 0.220$ & $166.0 \pm 9.0$ & & & \\
\hline $48 \mathrm{~b}$ & & $3.070 \pm 0.640$ & $2.330 \pm 0.410$ & $79.0 \pm 9.0$ & $3.060 \pm 0.210$ & $2.310 \pm 0.160$ & $169.0 \pm 9.0$ \\
\hline $48 \mathrm{c}$ & & $7.619 \pm 0.716$ & $1.042 \pm 0.085$ & $81.9 \pm 0.9$ & $2.300 \pm 1.000$ & $1.400 \pm 1.000$ & $119.0 \pm 9.0$ \\
\hline 62 & G19.27+0.1M1 & $0.485 \pm 0.019$ & $0.393 \pm 0.021$ & $134.6 \pm 7.2$ & $\ldots$ & $\ldots$ & $\ldots$ \\
\hline 63 & IRAS $18236-1205$ & $1.670 \pm 0.024$ & $0.970 \pm 0.011$ & $133.5 \pm 7.9$ & $1.510 \pm 0.028$ & $0.690 \pm 0.017$ & $132.5 \pm 9.4$ \\
\hline 64 & & $0.868 \pm 0.036$ & $0.738 \pm 0.026$ & $121.0 \pm 8.9$ & $0.499 \pm 0.079$ & $0.240 \pm 0.168$ & $109.0 \pm 9.0$ \\
\hline 65 & & $0.799 \pm 0.016$ & $0.713 \pm 0.013$ & $109.9 \pm 6.5$ & $0.394 \pm 0.040$ & $0.035 \pm 0.136$ & $93.2 \pm 9.2$ \\
\hline 73 & $\mathrm{G} 23.60+0.0 \mathrm{M} 1$ & $0.598 \pm 0.087$ & $0.365 \pm 0.035$ & $54.1 \pm 7.5$ & & & \\
\hline 74 & & $1.022 \pm 0.279$ & $0.397 \pm 0.061$ & $18.9 \pm 5.7$ & $0.878 \pm 0.356$ & $0.226 \pm 0.111$ & $15.0 \pm 9.0$ \\
\hline 83 & IRAS 18316-0602 & $1.069 \pm 0.105$ & $0.611 \pm 0.048$ & $178.4 \pm 5.4$ & $0.969 \pm 0.126$ & $0.451 \pm 0.096$ & $171.2 \pm 9.2$ \\
\hline 94 & & $0.946 \pm 0.284$ & $0.682 \pm 0.180$ & $57.0 \pm 9.0$ & $0.809 \pm 0.374$ & $0.576 \pm 0.511$ & $70.0 \pm 9.0$ \\
\hline 95 & & $0.455 \pm 0.081$ & $0.388 \pm 0.059$ & $16.0 \pm 9.0$ & $\ldots$ & $\ldots$ & $\ldots$ \\
\hline 96 & $\mathrm{G} 24.08+0.0 \mathrm{M} 2$ & $0.503 \pm 0.021$ & $0.407 \pm 0.023$ & $27.4 \pm 7.8$ & $\ldots$ & $\ldots$ & $\ldots$ \\
\hline 110 & $\mathrm{G} 24.33+0.1 \mathrm{M} 1$ & $0.528 \pm 0.018$ & $0.350 \pm 0.020$ & $33.4 \pm 3.3$ & $\ldots$ & $\ldots$ & $\ldots$ \\
\hline 136 & $\mathrm{G} 24.60+0.01 \mathrm{M} 2$ & $0.685 \pm 0.068$ & $0.492 \pm 0.068$ & $78.0 \pm 9.0$ & & & $\ldots$ \\
\hline 137 & $\mathrm{G} 34.43+0.2 \mathrm{M} 3$ & $1.025 \pm 0.060$ & $0.797 \pm 0.070$ & $61.0 \pm 9.0$ & $0.899 \pm 0.085$ & $0.715 \pm 0.108$ & $123.8 \pm 3.9$ \\
\hline 143 & IRAS $19095+0930$ & $0.572 \pm 0.012$ & $0.371 \pm 0.012$ & $50.0 \pm 1.9$ & $0.269 \pm 0.034$ & $0.181 \pm 0.058$ & $94.0 \pm 9.0$ \\
\hline
\end{tabular}



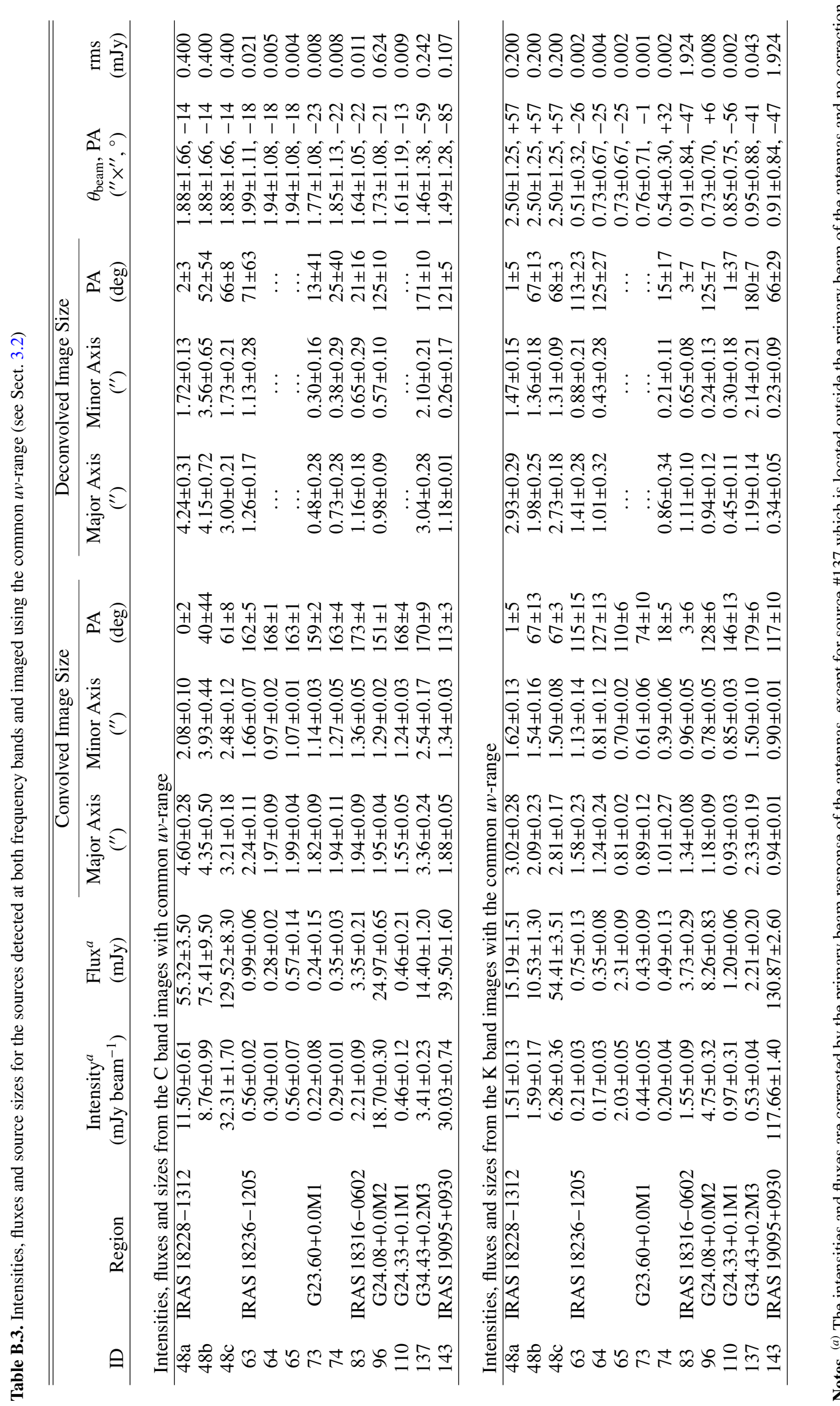

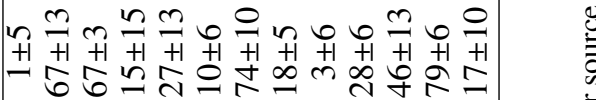

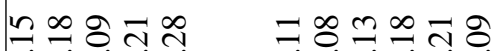

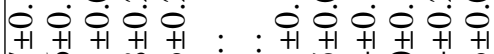
f

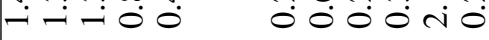

กิ่

it if o

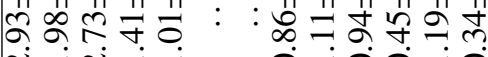

i-i - -

o- $0 \dot{0}-0$

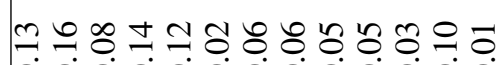

in $+\begin{aligned} & 1 \\ & 1\end{aligned}+\begin{aligned} & 1 \\ & 0\end{aligned}$

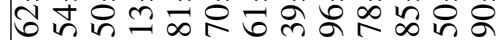

计

要

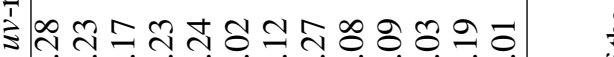

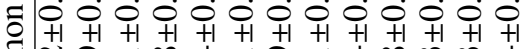

రิ \&े $\infty$ in

m तi

$\stackrel{2}{\Xi}$

ที

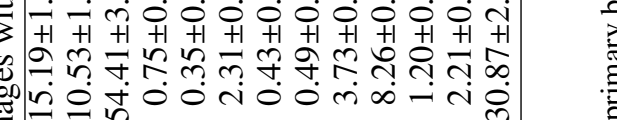

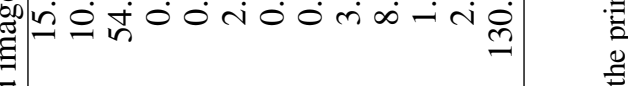

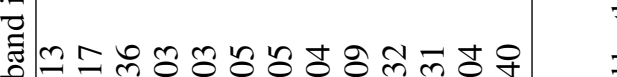

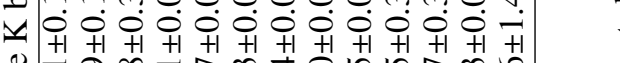

E n

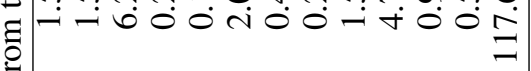

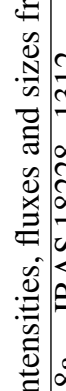

告

苍

শ

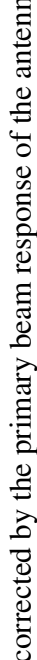

$\stackrel{\square}{\square}$

龸

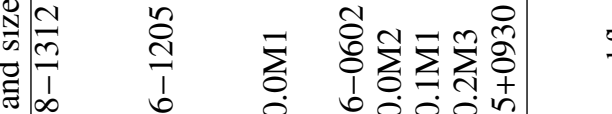

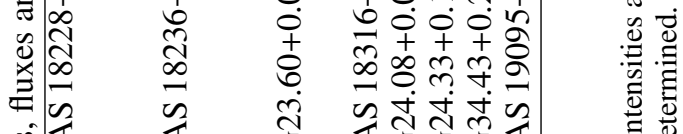

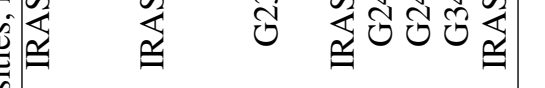

过

ङ

它蓄

Article number, page 27 of 40 
A\&A proofs: manuscript no. aanda
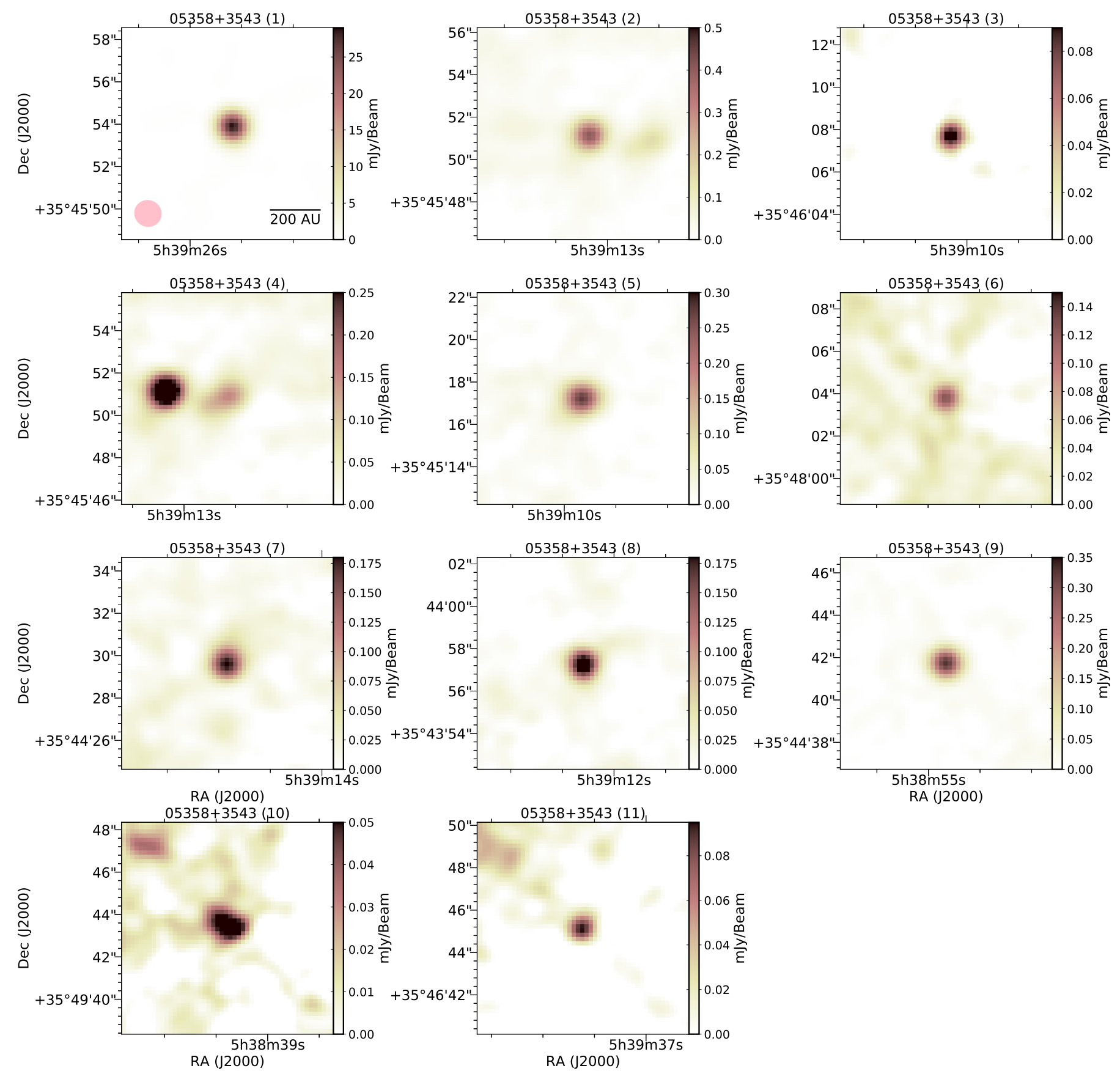

Fig. B.1. Close-up views of the $\mathrm{C}$ band (color scale image) and $\mathrm{K}$ band (contours) continuum images for the sources listed in Table B.1. Maps for the sources detected in region IRAS $05358+3543$. 
Kavak et al.: A search for radio jets from massive young stellar objects
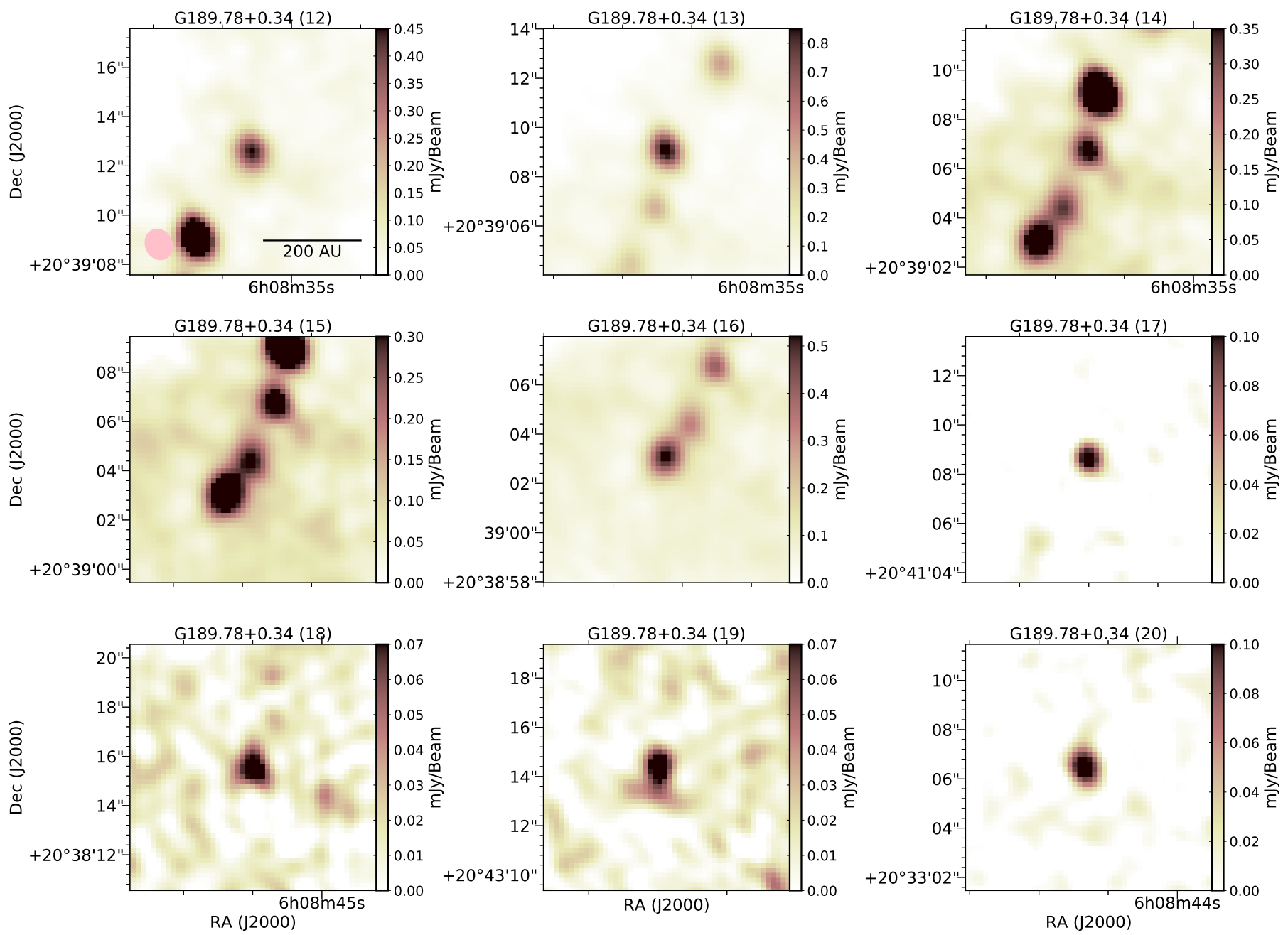

Fig. B.2. Close-up views of the $\mathrm{C}$ band (color scale image) and $\mathrm{K}$ band (contours) continuum images for the sources listed in Table B.1. Maps for the sources detected in region G189.78+0.34.
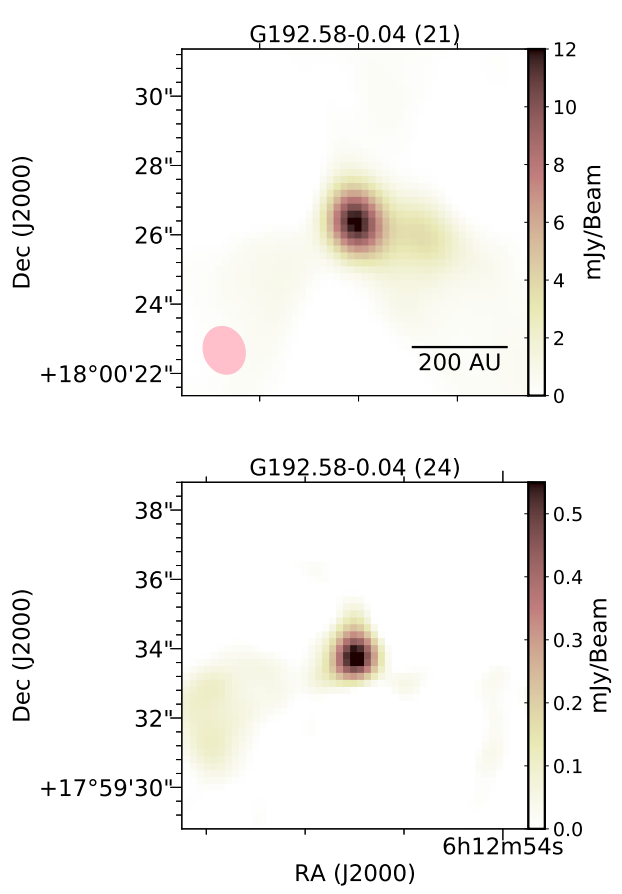
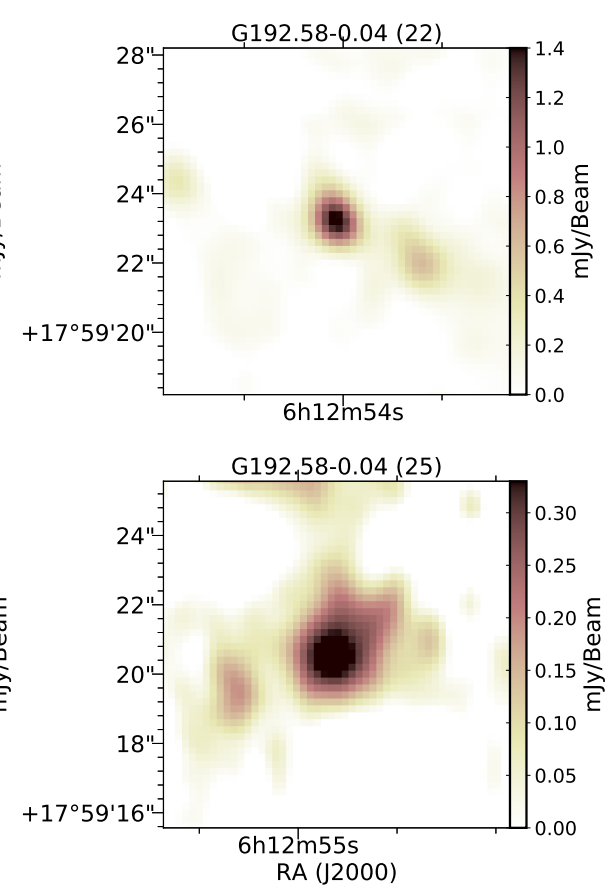
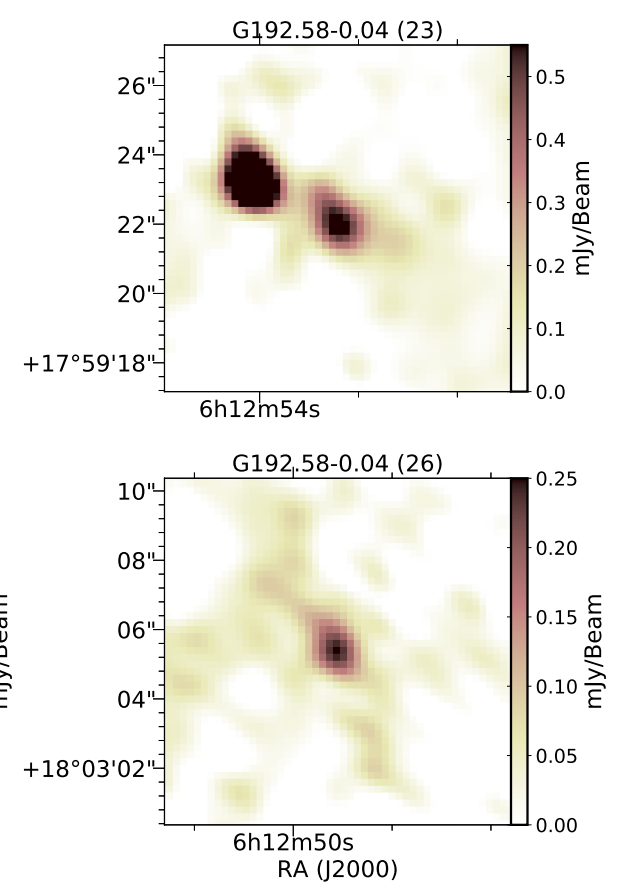

Fig. B.3. Close-up views of the $\mathrm{C}$ band (color scale image) and $\mathrm{K}$ band (contours) continuum images for the sources listed in Table B.1. Maps for the sources detected in region G192.58-0.04. 

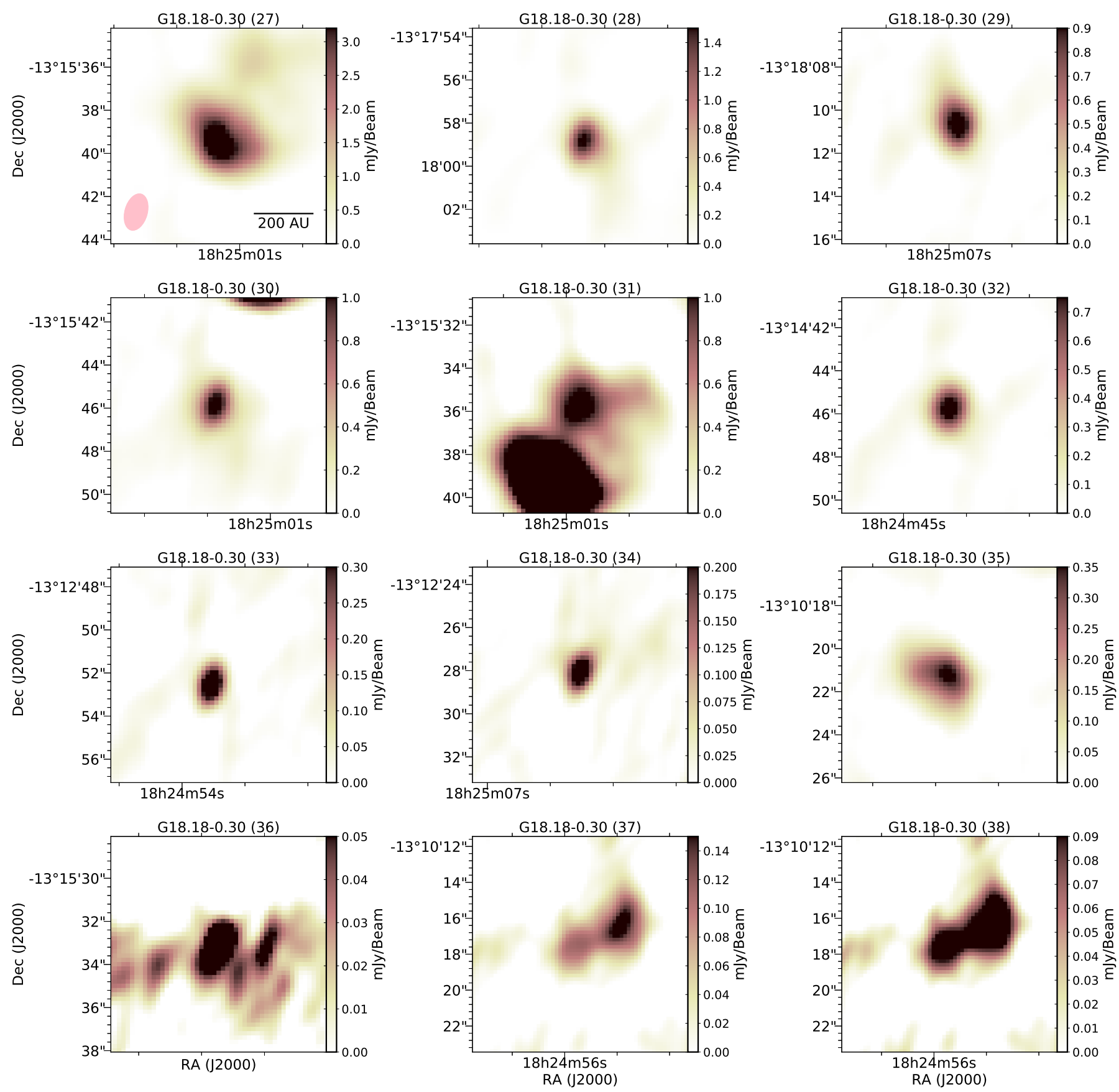

Fig. B.4. Close-up views of the $\mathrm{C}$ band (color scale image) and $\mathrm{K}$ band (contours) continuum images for the sources listed in Table B.1. Maps for the sources detected in region G18.18-0.30. 
Kavak et al.: A search for radio jets from massive young stellar objects
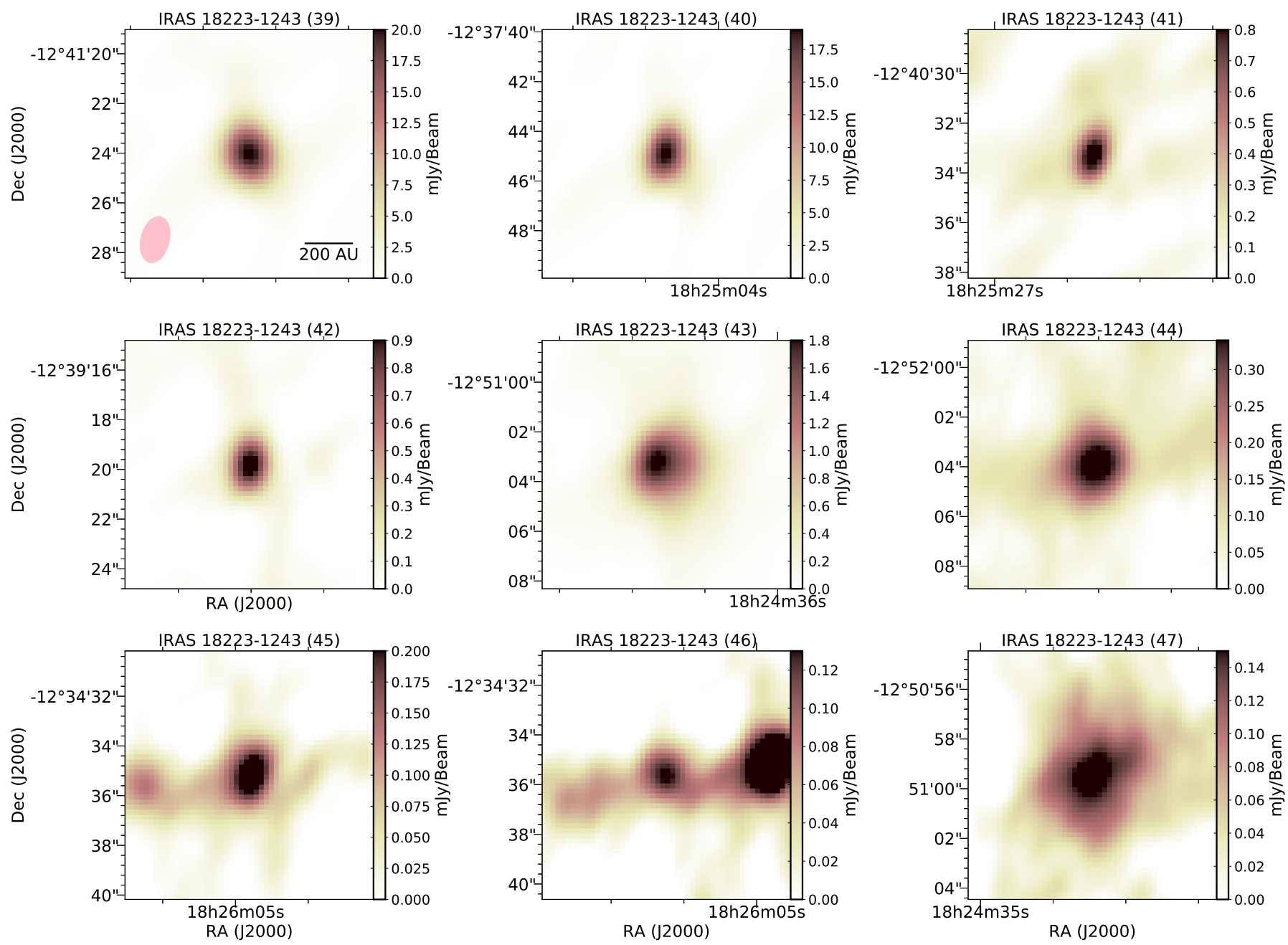

Fig. B.5. Close-up views of the $\mathrm{C}$ band (color scale image) and $\mathrm{K}$ band (contours) continuum images for the sources listed in Table B.1. Maps for the sources detected in region IRAS 18223-1243.
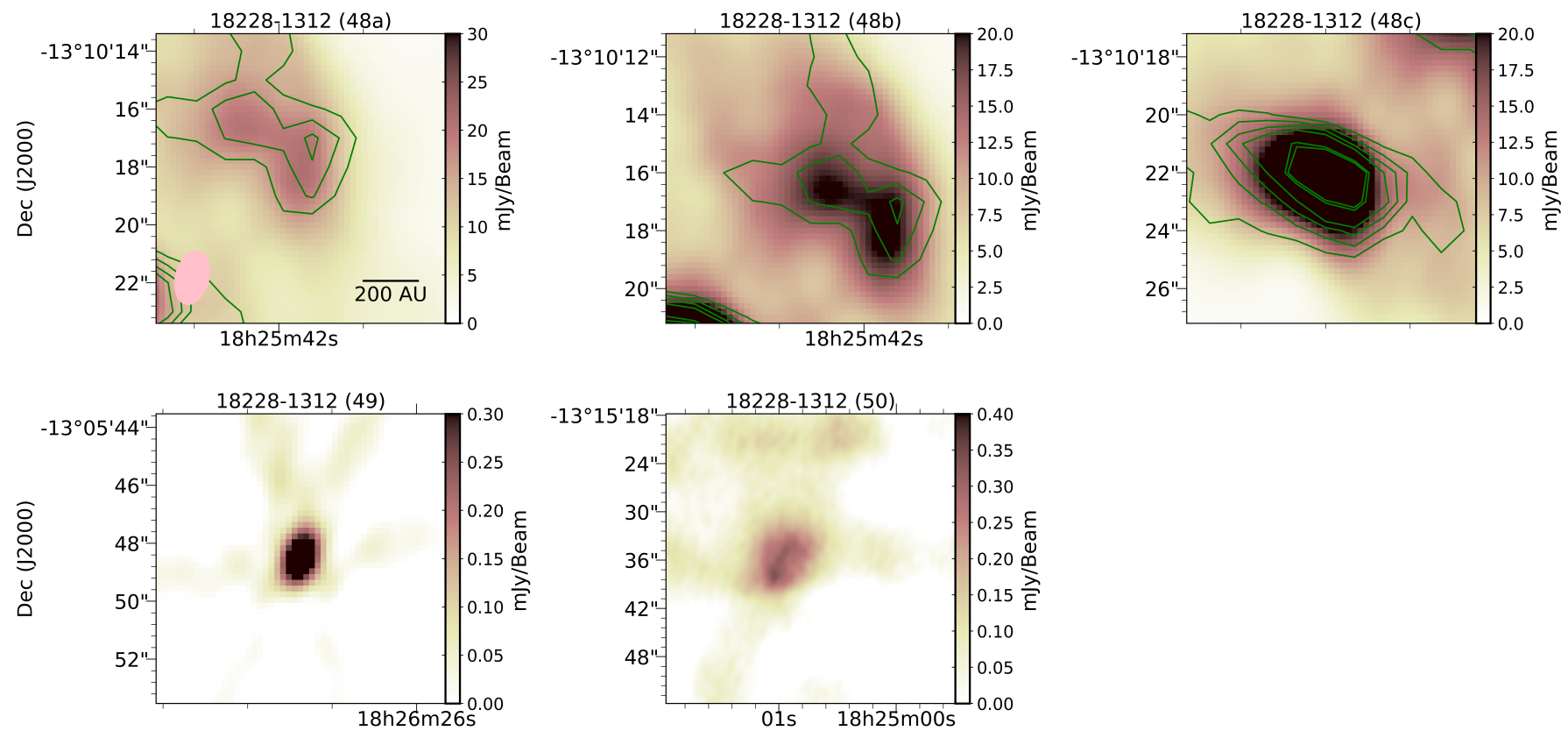

Fig. B.6. Close-up views of the $\mathrm{C}$ band (color scale image) and $\mathrm{K}$ band (contours) continuum images for the sources listed in Table B.1. Maps for the sources detected in region IRAS 18228-1312. 

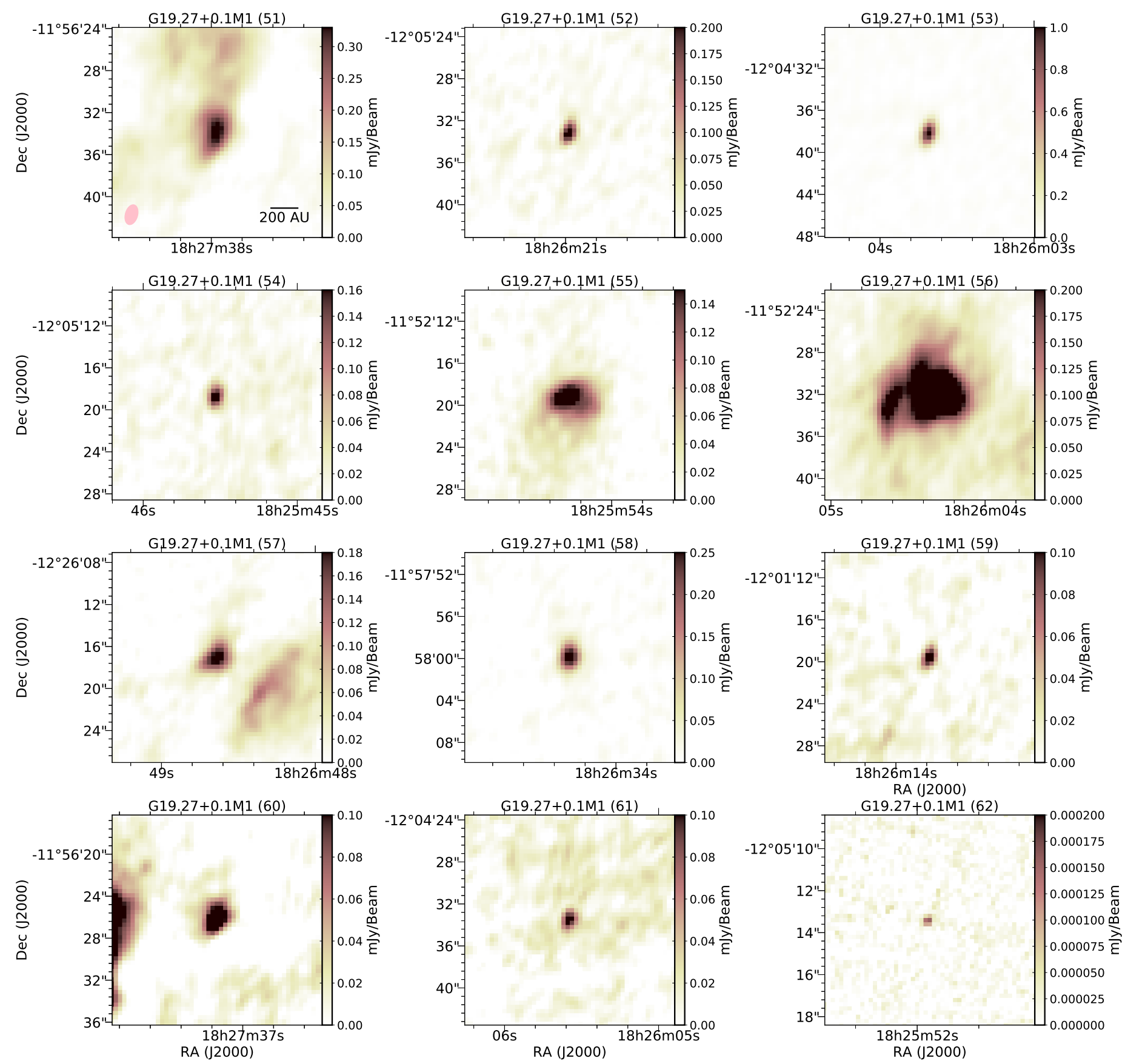

Fig. B.7. Close-up views of the $\mathrm{C}$ band (color scale image) and $\mathrm{K}$ band (contours) continuum images for the sources listed in Table B.1. Maps for the sources detected in region G19.27+0.1 M1. 
Kavak et al.: A search for radio jets from massive young stellar objects
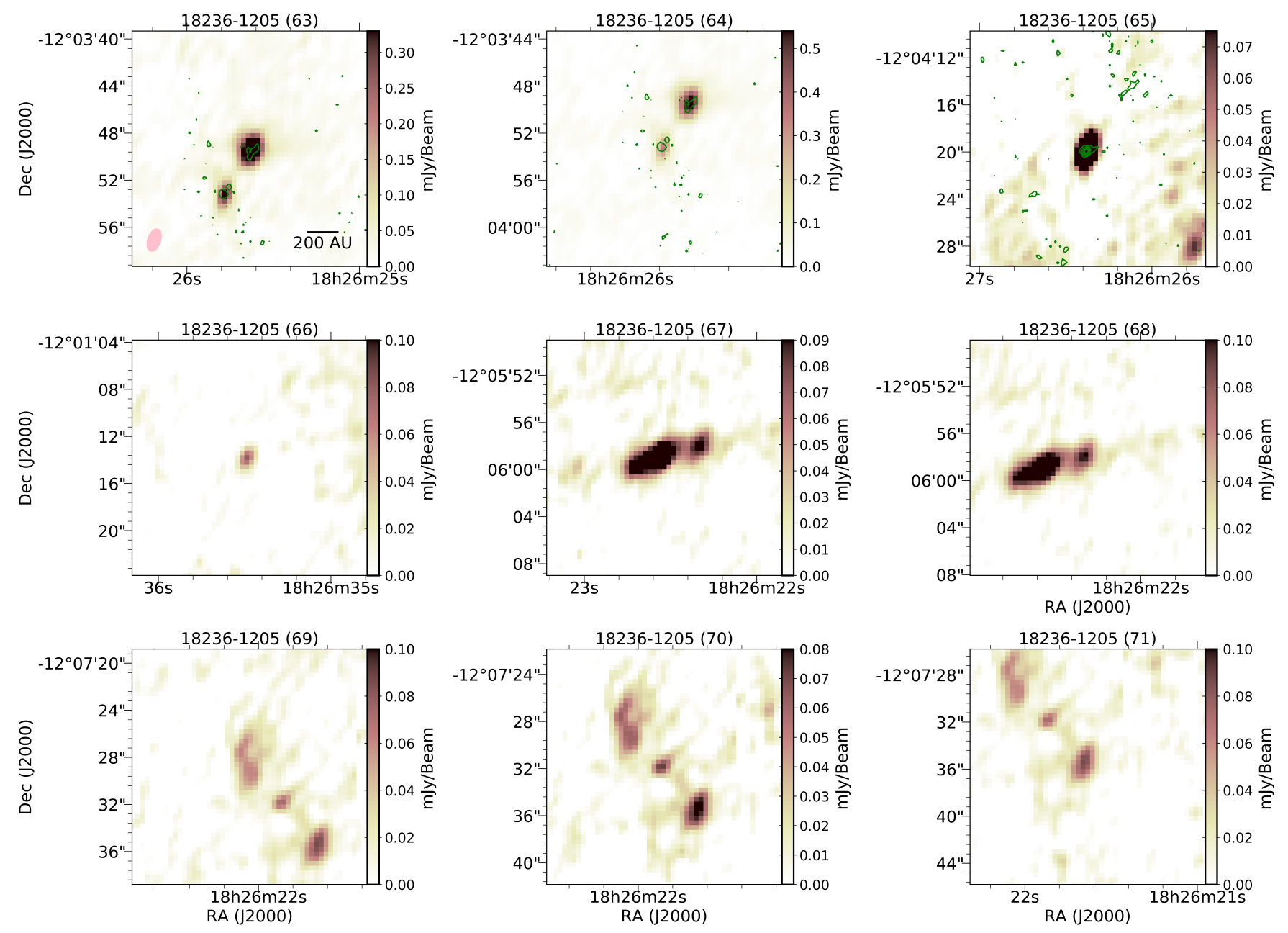

Fig. B.8. Close-up views of the $\mathrm{C}$ band (color scale image) and $\mathrm{K}$ band (contours) continuum images for the sources listed in Table B.1. Maps for the sources detected in region IRAS 18236-1205. 

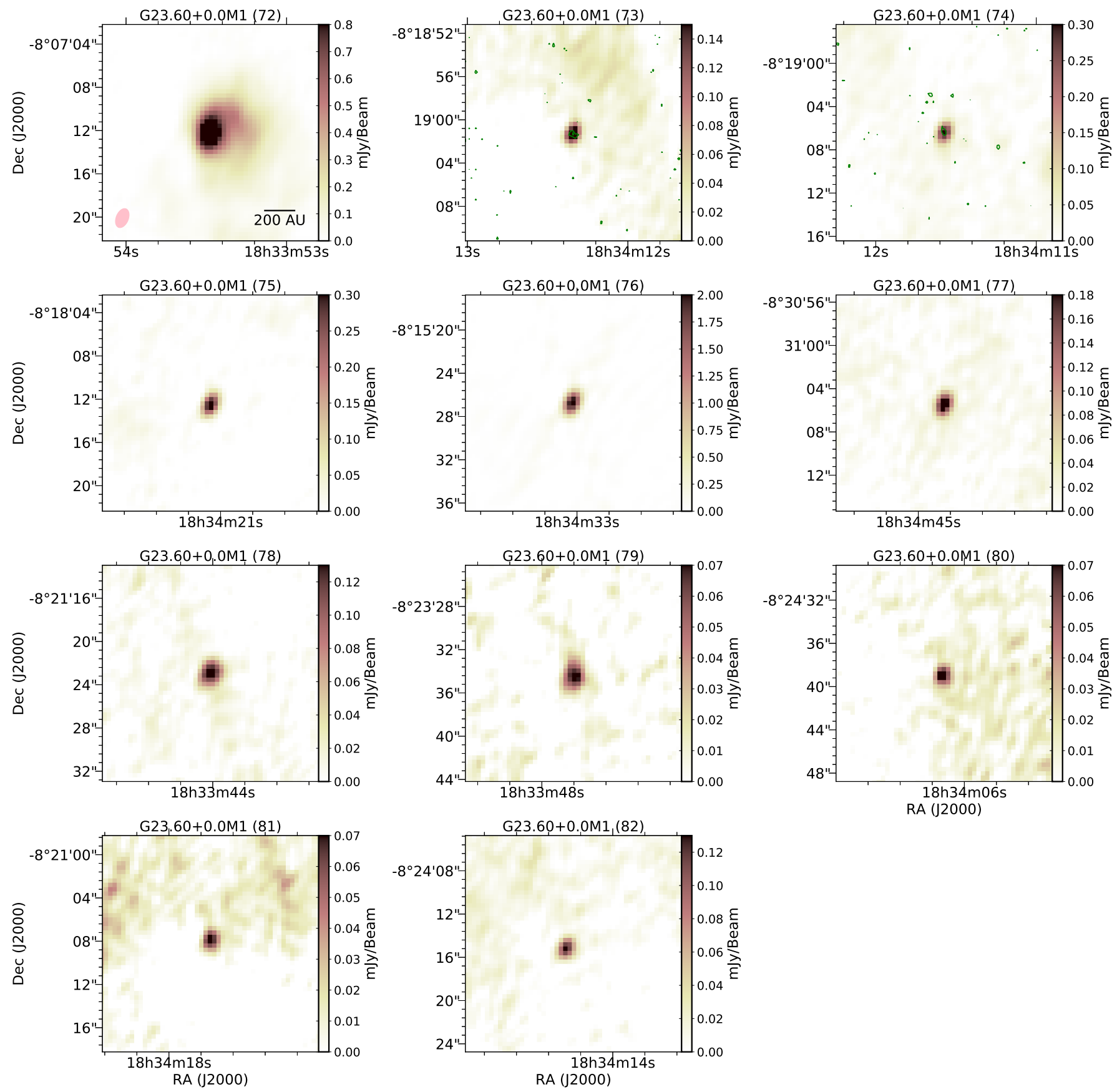

Fig. B.9. Close-up views of the $\mathrm{C}$ band (color scale image) and $\mathrm{K}$ band (contours) continuum images for the sources listed in Table B.1. Maps for the sources detected in region G23.60+0.0 M1. 
Kavak et al.: A search for radio jets from massive young stellar objects
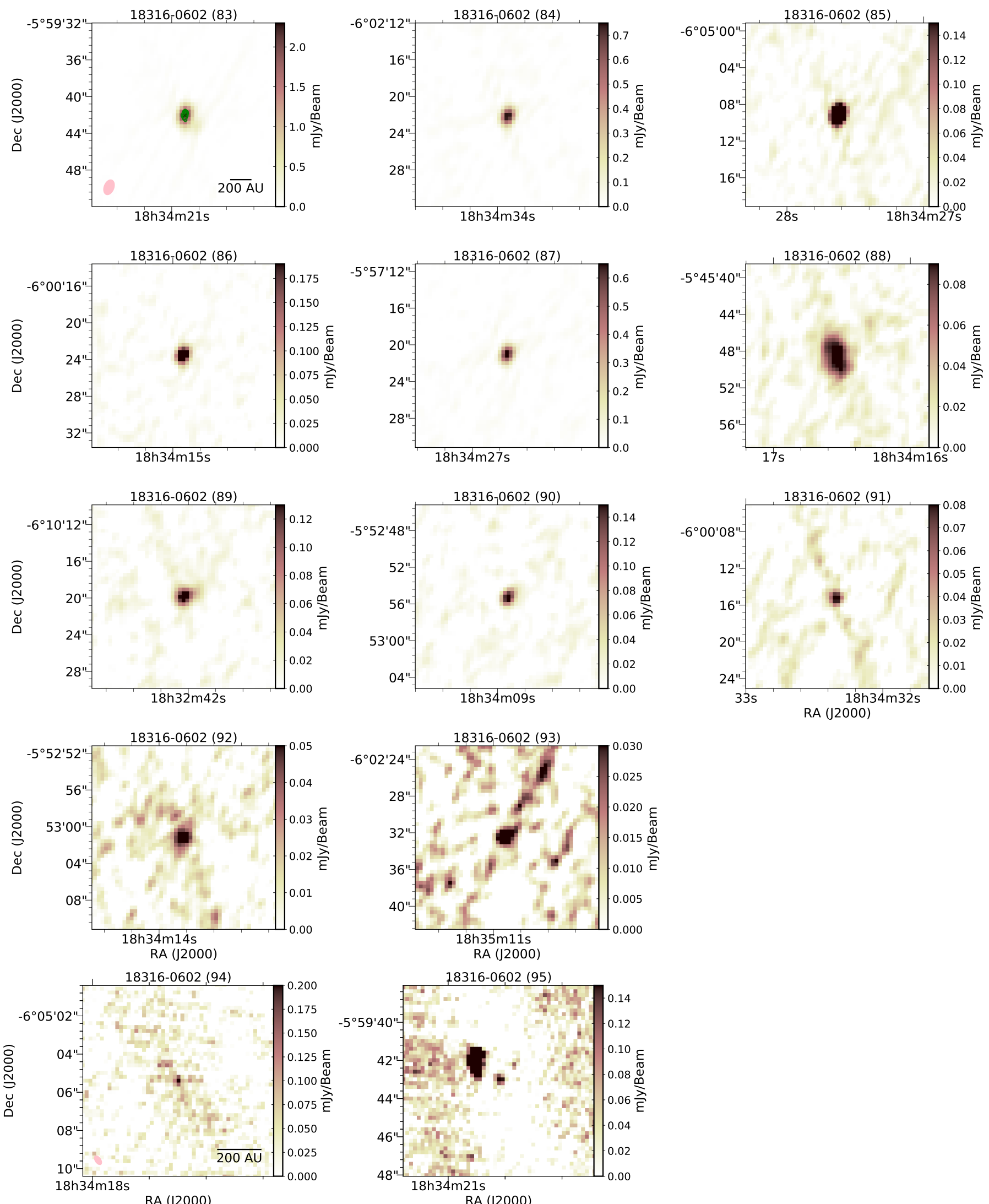

Fig. B.10. Close-up views of the $\mathrm{C}$ band (color scale image) and $\mathrm{K}$ band (contours) continuum images for the sources listed in Table B.1. The image of sources \#94 and \#95 correspond to the K band maps. Maps for the sources detected in region IRAS 18316-0602. 

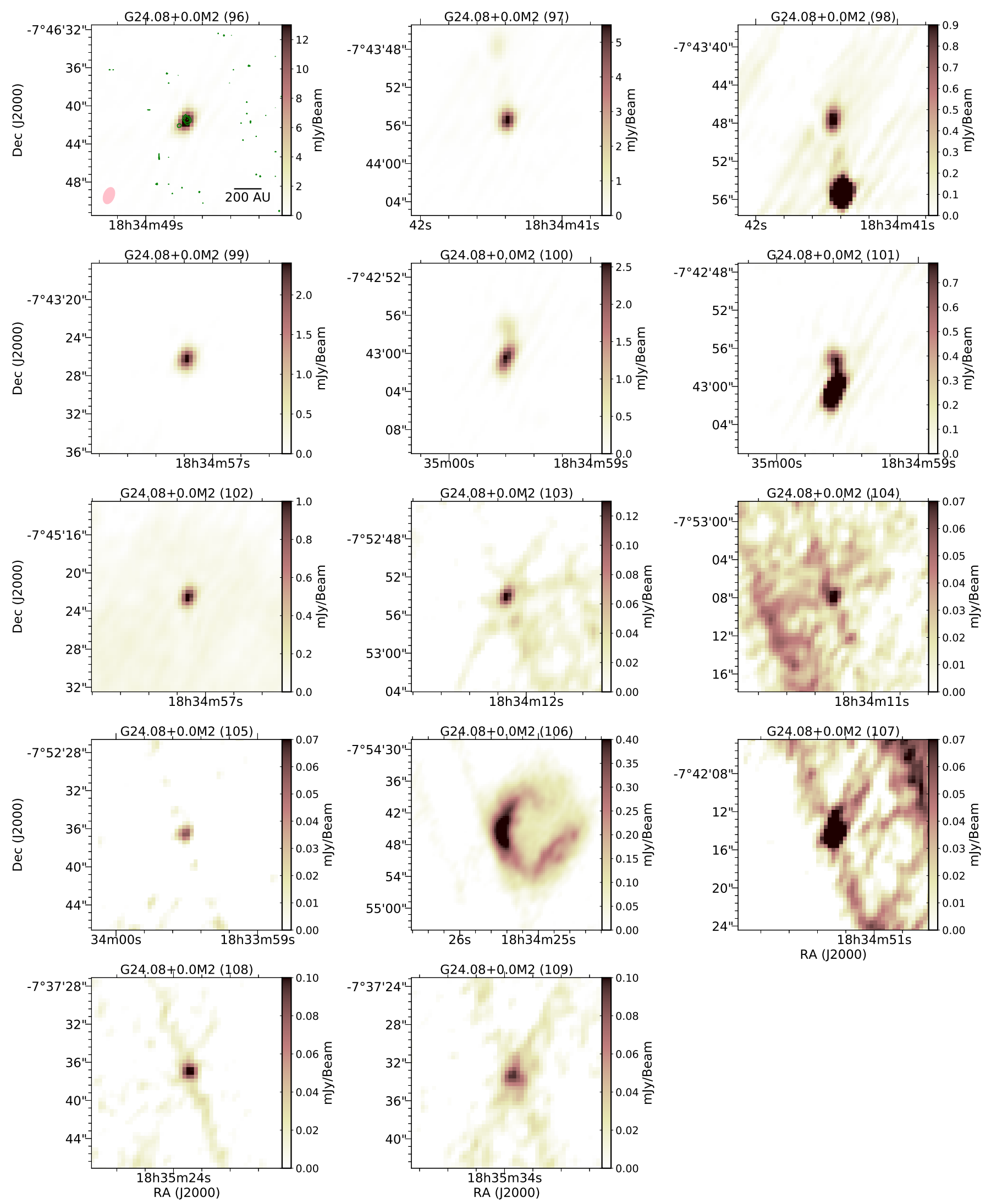

Fig. B.11. Close-up views of the $\mathrm{C}$ band (color scale image) and $\mathrm{K}$ band (contours) continuum images for the sources listed in Table B.1. Maps for the sources detected in region G24.08+0.0 M2. 
Kavak et al.: A search for radio jets from massive young stellar objects
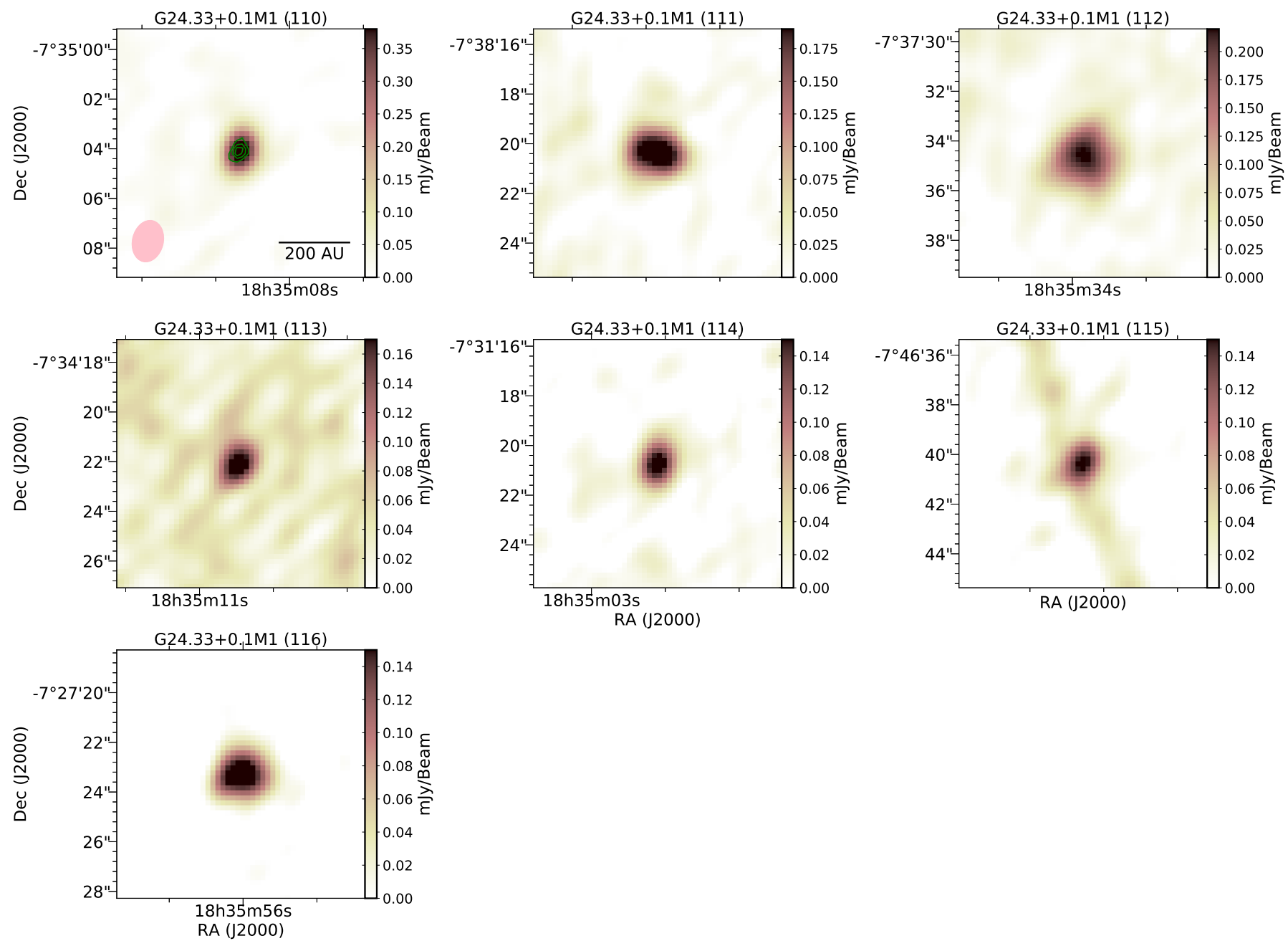

Fig. B.12. Close-up views of the $\mathrm{C}$ band (color scale image) and $\mathrm{K}$ band (contours) continuum images for the sources listed in Table B.1. Maps for the sources detected in region G24.33+0.1 M1. 

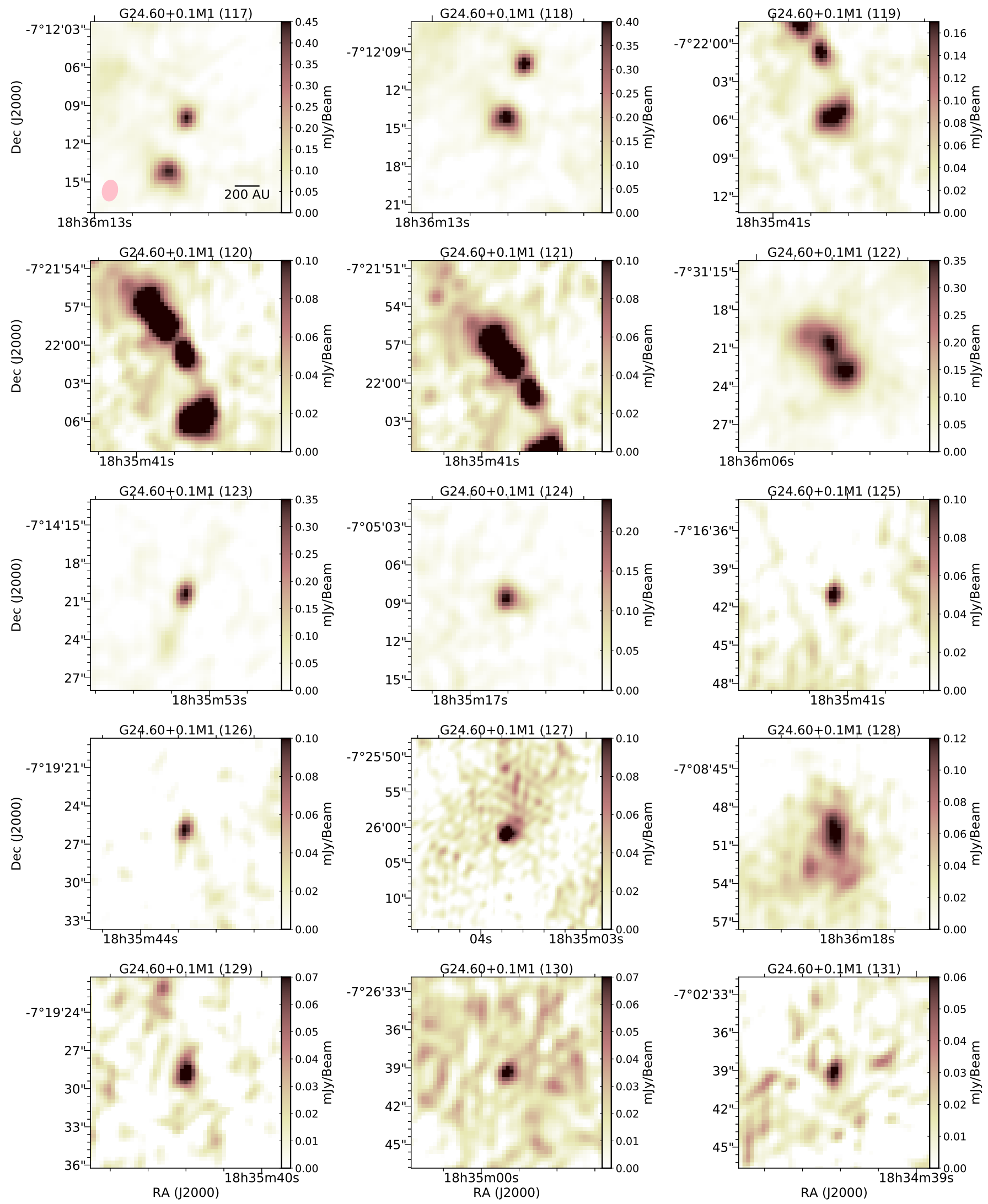

Fig. B.13. Close-up views of the $\mathrm{C}$ band (color scale image) and $\mathrm{K}$ band (contours) continuum images for the sources listed in Table B.1. Maps for the sources detected in region G24.60+0.1 M1. 
Kavak et al.: A search for radio jets from massive young stellar objects
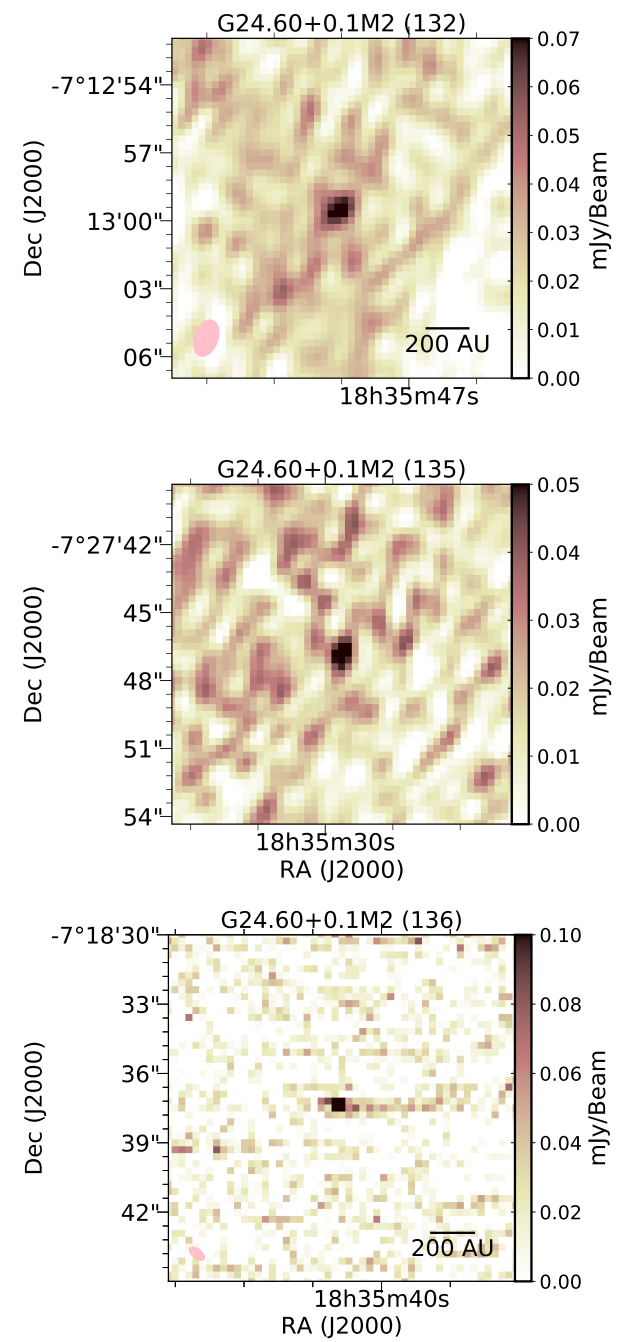
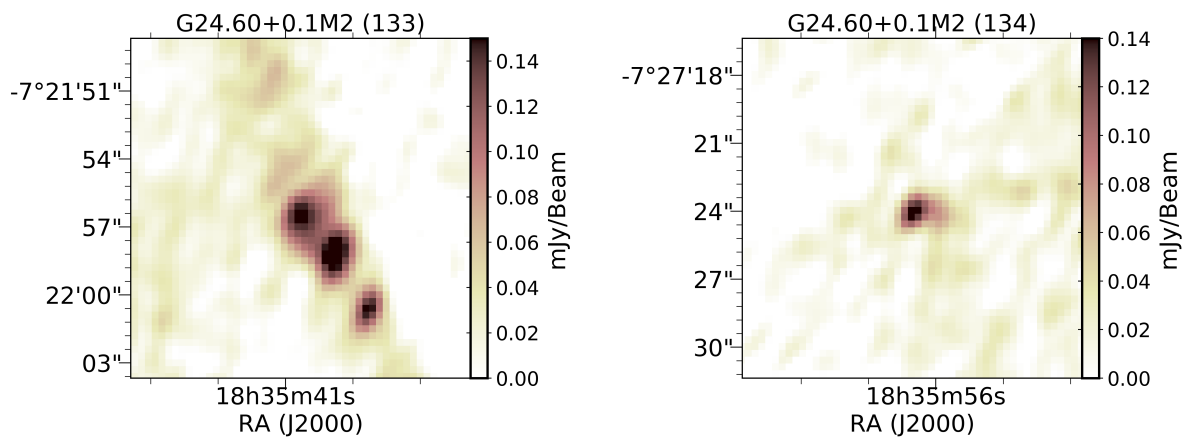

Fig. B.14. Close-up views of the $\mathrm{C}$ band (color scale image) and $\mathrm{K}$ band (contours) continuum images for the sources listed in Table B.1. The image of source \#136 corresponds to the K band map. Maps for the sources detected in region G24.60+0.1 M2. 

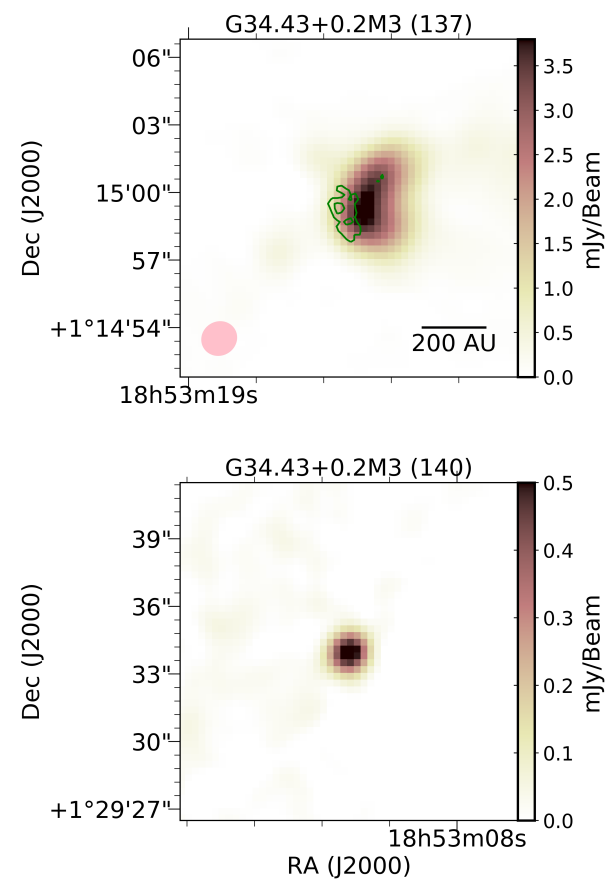
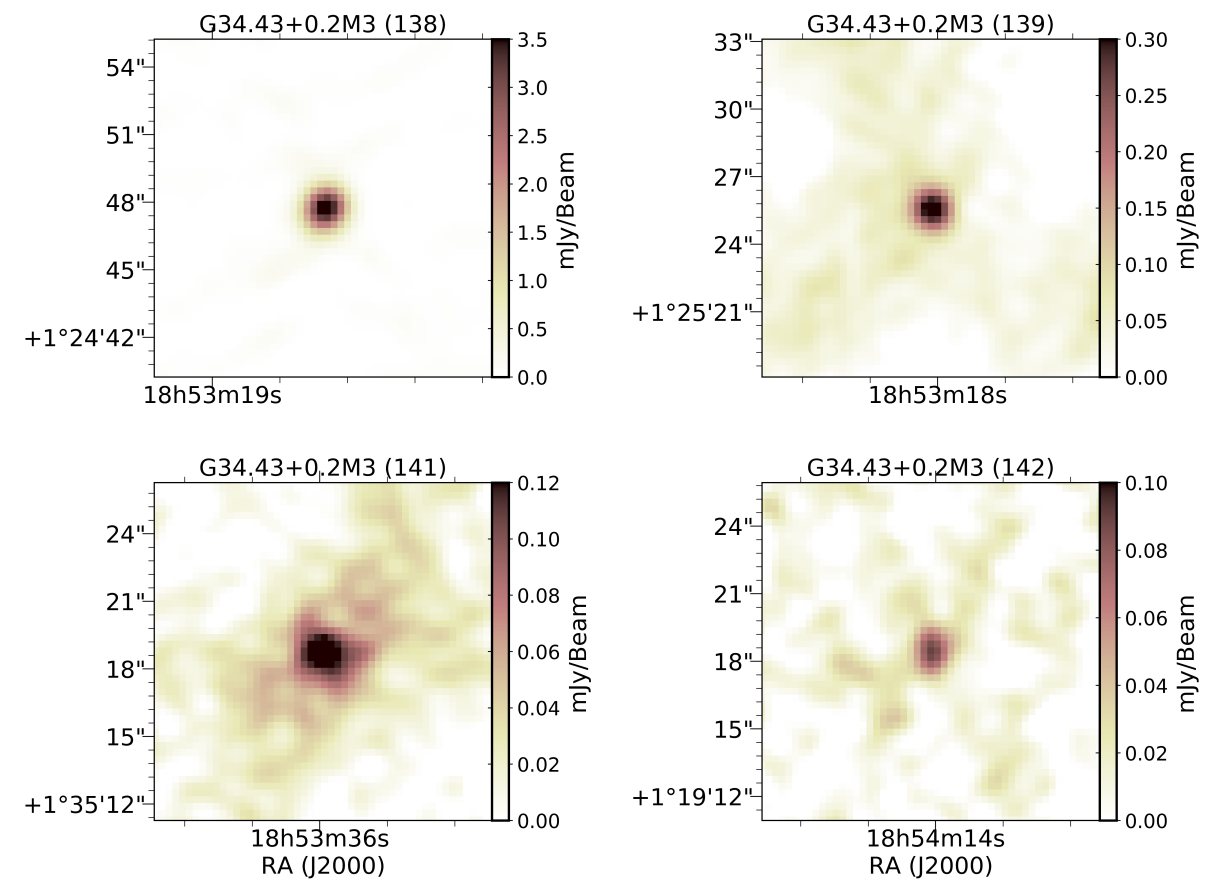

Fig. B.15. Close-up views of the $\mathrm{C}$ band (color scale image) and $\mathrm{K}$ band (contours) continuum images for the sources listed in Table B.1. Maps for the sources detected in region $\mathrm{G} 34.43+0.2 \mathrm{M} 3$.
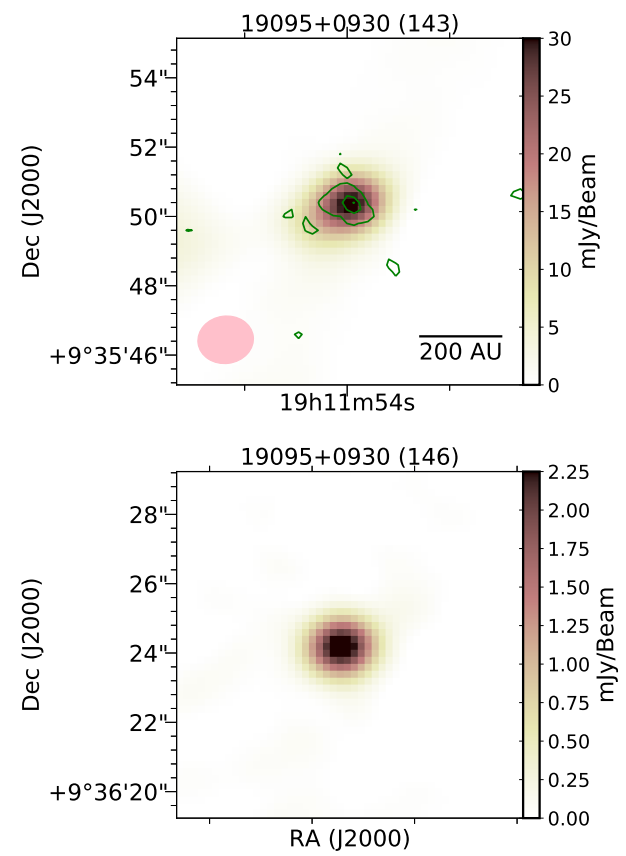
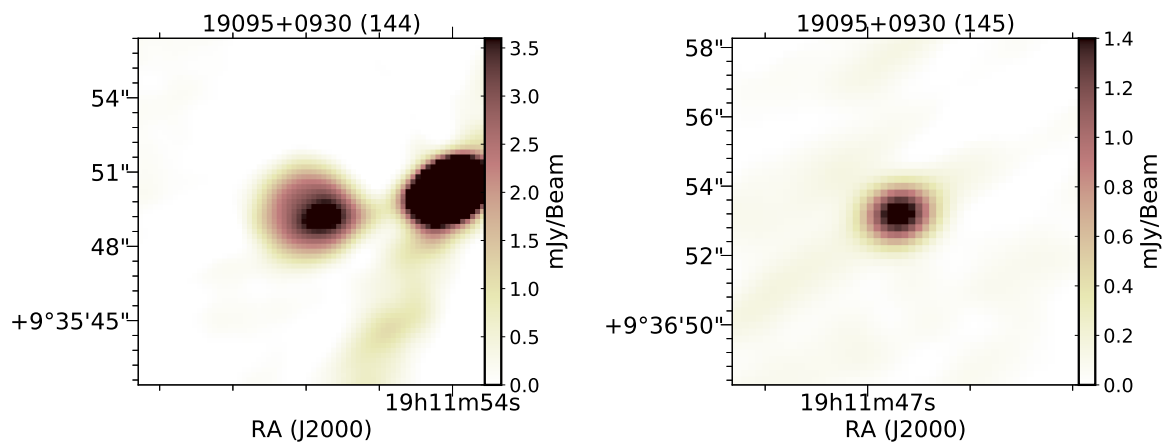

Fig. B.16. Close-up views of the $\mathrm{C}$ band (color scale image) and $\mathrm{K}$ band (contours) continuum images for the sources listed in Table B.1. Maps for the sources detected in region IRAS 19095+0930. 